\title{
7
}

\section{Technical Annexes}

\section{Contents}

7.1 Humanitarian Programs............................ 141

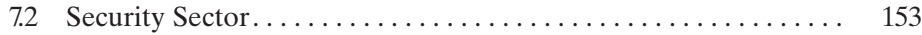

7.3 Health Sector...................................... 158

Core Disciplines in Disaster Health . . . . . . . . . . . . . . . 161

Primary Health Care Programs . . . . . . . . . . . . . . . . 162

Disease Prevention .............................. 162

Clinical Facilities ............................... 164

Reproductive Health ........................... 165

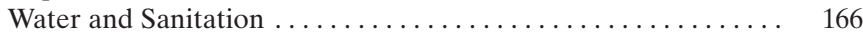

Food and Nutrition $\ldots \ldots \ldots \ldots \ldots \ldots \ldots \ldots \ldots \ldots \ldots \ldots \ldots \ldots \ldots \ldots$

Chemical Weapons . . . . . . . . . . . . . . . . . . . . . . . . 181

Epi Methods ................................. 184

7.4 Tropical Medicine .............................. 187

Tropical Infectious Diseases - Vector-borne and Zoonotic. . . . . 196

Tropical Infectious Diseases-Non-vector-borne........... 215

7.5 Epidemic Preparedness and Response.................. 239

7.6 Communicable Disease Control . . . . . . . . . . . . . . . . . . . . 242

Diarrhea..................................... 244

Influenza................................... 257

Malaria .................................... 263

Measles...................................... 267

Meningitis.................................. 269

Viral Hemorrhagic Fever........................ 272

(C) David A. Bradt 2020

D. A. Bradt, C. M. Drummond, Pocket Field Guide

for Disaster Health Professionals, https://doi.org/10.1007/978-3-030-04801-3_7 


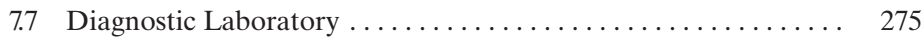

Indications, Laboratory Tests, and Expected Availability ..... 276

Specimen Handling. . . . . . . . . . . . . . . . . . . . . . 278

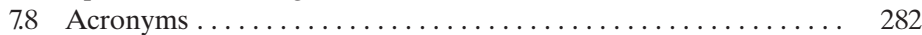

\section{Overview}

This section provides guidance on technical issues in the health sector. The annexes contain compilations of frequently used reference information.

- Humanitarian programs-contains conceptual frameworks on global clusters, relief programs, humanitarian financing, and early recovery.

- Security sector-contains key definitions from the Rome Statute of the International Criminal Court

- Health sector-contains a broad range of core health technical information including environmental classification of water and excreta-related diseases, disease prevention measures, water treatment end points, anthropometric classifications, micronutrient deficiency states, management of chemical weapon exposures, and epi methods.

- Tropical medicine-contains clinical summaries of tropical infectious diseases with details on disease vector and host, clinical presentation, diagnostic lab tests, clinical epidemiology, and therapy.

- Epidemic preparedness and response-contains core principles of epidemic preparedness and response.

- Communicable disease control-contains an overview of selected communicable diseases of epidemic potential including diarrhea, influenza, malaria, measles, meningitis, and viral hemorrhagic fever.

- Diagnostic laboratory-contains guidance on lab specimen handling and testing.

- Acronyms - contains acronyms commonly used in disaster management and humanitarian assistance. 


\section{Document 7.1 \\ HUMANITARIAN PROGRAMS}

\section{HUMANITARIAN ASSISTANCE VALUES AND GOALS}

\section{A. Core Values}
1. Humanity
2. Impartiality
3. Neutrality
4. Independence

B. Goals
1. Save lives
2. Alleviate suffering
3. Reduce economic and social impact of disaster
4. Undertake principled humanitarian action
5. Provide integrated multi-sector assistance and protection to the most vulnerable
6. Maintain peace and security
7. Uphold law and order (host government)
8. Implement durable solutions (UNHCR)
a. repatriation
b. local integration
c. resettlement
9. Mitigate hazards
10. Build back better
11. Support community resilience, social cohesion, and early recovery

II. GLOBAL CLUSTERS AND LEADS (IASC [1,2])

A. Technical Areas
1. Nutrition
UNICEF
2. Health
WHO
3. Water/sanitation
UNICEF
4. Emergency shelter
IDPs from conflict
UNHCR
5. Food security

B. Cross-cutting Areas

1. Camp coordination IDPs from conflict
disaster situations

UNHCR

2. Protection

IOM

3. Early recovery

UNHCR

4. Education

UNDP

UNICEF/SC

C. Common Service Areas

1. Logistics

2. Emergency telecoms 
III. SECTORS AND THEMES

\section{A. Sectors}

1. Coordination

2. Protection and Registration

3. Security and Demobilization

4. Logistics

5. Site Planning

6. Water and Sanitation

7. Food Aid

8. Agriculture

9. Non-food Aid (household support)

10. Shelter

11. Health

12. Rehabilitation

13. Education and Training

14. Economic Recovery and Community Development

15. Durable Solutions

B. Cross-cutting Themes (IASC)

1. Human Rights \& Protection

2. Gender

3. HIV/AIDS

4. Environment

\section{Themes Per Donor Grants Guidelines}

1. Artisanal Production

2. Capacity Building/Training

3. Cash Distribution

4. Cash for Work

5. Children

6. Conflict Resolution

7. Gender Relations

8. HIV-AIDS

9. Host Communities

10. Host Government

11. IDPs

12. Information Systems

13. Infrastructure Rehabilitation

14. Livelihoods/Income Generation

15. Market Rehabilitation

16. Micro-Finance/Micro-Credit

17. Nomads/Pastoralists

18. Protection Mainstreaming

19. Returnees

20. Slavery/Trafficking

21. Vouchers 


\section{RELIEF PROGRAMS}

\section{A. Programming Principles}

1. Address identified needs in underserved areas

2. Encourage local participation

3. Integrate beneficiaries into program planning

4. Collaborate with all stakeholders

5. Coordinate with all implementing partners

6. Plan comprehensive approaches

7. Develop community-based programs

8. Make inter-sectoral linkages (sector-wide approaches)

9. Use existing resources

10. Leverage outside resources of donors and private sector

11. Build on existing platforms

12. Apply international best practices

13. Target vulnerable populations

14. Focus on high impact activities

15. Ensure equitable access to services

16. Provide assistance acceptable to beneficiaries

17. Implement with cultural sensitivity

18. Reduce the local burden of disease

19. Enhance capacities

20. Reduce vulnerabilities

21. Alleviate poverty

22. Avoid dependency

23. Foster sustainable development

24. Support governmental priorities

25. Operate cost-effectively, transparently, and accountably

\section{B. Programming Approach}

1. Prioritize a core package of life-saving interventions in geographically targeted areas

2. Support humanitarian protection throughout the response

3. Provide flexible programming that lets partners adapt to new demands in new areas

4. Adopt market-based modalities

5. Enhance the humanitarian architecture

6. Support livelihoods programming that complements livesaving assistance when safe to do so to

7. Support early recovery and transition to development

C. Keys to Emergency Relief (M Toole, Burnet Institute, Melbourne, Australia, unpublished)

1. Intervene early

2. Support, not undermine, community coping strategies

3. Prevent communities from migrating

4. Avoid establishing large refugee camps

5. Establish a health information system 
6. Ensure resources provided do not further divide communities

7. Focus on disease prevention

8. Work through existing structures and institutions

9. Insist that women control the distribution of relief supplies

10. Ensure open communication and coordination

\section{Implementation Mechanics}

1. Reallocation of existing local \& national resources

2. Revision/refinement of present (medical) practices

a. use of essential drugs

b. use of standardized case management

c. replacement of dated practices

3. Financial assistance (critical funding)

4. Technical assistance (critical skills)

a. complement donated goods

b. support appropriate technology

c. develop information systems

5. Material assistance (critical goods)

a. essential drugs and vaccines

b. consumable supplies

c. other stated needs of health authorities

6. Direct service provision (substitution)

7. Coordination of external assistance

NB cooperation $=$ shared goals; coordination $=$ shared tasks;

collaboration $=$ shared resources

a. identification and registration of actors

b. actor/area/activity (who/what/where) matrix

c. gap analysis and priority setting

d. establishment of rapid response mechanism

e. gap filling via complementary inputs linking iNGOs \& local

NGOs to local health authorities

(1) technical

(2) material

(3) financial

f. identification of deliverables and timetables

g. consolidated reporting and dissemination

(1) group terms of reference

(2) meeting minutes

(3) epidemiology updates

(4) health sitreps

(5) component analysis

(6) field documentation (toolkit) for new arrivals

8. Capacity building of host authorities

9. Civil society partnership and support

a. organize community leaders

b. encourage gender mainstreaming

c. encourage privatization

d. discourage entitlements

10. Advocacy

11. Transition to early recovery 


\section{E. Strategy for Livelihood/Economic Relief}

1. Restore productive assets (supply side interventions)

a. in-kind donations (eg food, seeds, tools, fishing nets, etc)

b. types of community projects in food-for-assets programs

(1) natural resources development

(a) water harvesting

(b) soil conservation

(2) restoration of agri(aqua)culture potential

(a) irrigation systems

(b) seed systems

(3) infrastructure rehabilitation
(a) schools
(b) market places
(c) community granaries
(d) warehouses
(e) roads
(f) bridges

(4) diversification of livelihoods

(a) training and experience sharing

2. Increase individual purchasing power
a. cash distribution
b. cash for work (cash for assets)
c. vouchers
d. micro-credit
e. job fairs
f. artisanal production
g. livelihoods/income generation

3. Support market resumption
a. market rehabilitation
b. infrastructure rehabilitation
c. micro-finance institutions

\section{F. Strategy for Early Recovery}

Goals - protect what's left (1 month), restore the system (6 months), improve the system (6 months)

1. Adopt systems approach

a. health services

b. health workforce

c. medical logistics (drugs, vaccines, equipment, supplies, \& technology)

d. health information system

e. health financing

f. leadership \& governance

2. Phase in assistance to beneficiaries

a. technical assistance

b. material assistance

(1) food

(2) non-food items 
c. financial assistance

(1) cash grants

(2) cash for work

(3) microfinance (loans)

(4) livelihood/income generation

3. Ensure responsible resource management

a. human resources management

(1) incident management command and control

(2) team structure and function

(3) staff selection

(a) internationals

(b) homologues

(4) field activities
(a) briefing
(b) meetings and reports
(c) debriefing

(5) operations support
(a) comms
(b) transport
(c) office
(d) food and lodging

(6) personal health maintenance and morale

b. material resources management

c. financial resources management

d. supervision

e. monitoring and evaluation

4. Scale up coverage of priority health interventions

5. Address bottlenecks of the disrupted health system (otherwise temporary solutions become permanent)

6. Protect essential public health infrastructures

7. Build capacity of local authorities with focus on sustainable systems

a. technical oversight-hiring of local experts

b. material assistance-production of key commodities

c. financial assistance

8. Provide incentives for host government

9. Support host country non-beneficiary population

10. Find new partners in the development community

11. Use health Sustainable Development Goals as targets for recovery activities

12. Seek opportunities and develop mechanisms for transition and phase out

\section{G. Program Constraints and Failures}

1. Programmatic constraints

a. staff
(1) western trained
(2) hospital-based
(3) resource intensive 
(4) technology dependent

(5) procedurally oriented

(6) invasive

(7) monolingual

(8) hazard naïve

b. supervision

(1) limited responsibility

(2) limited authority

(3) limited accountability

c. projects
(1) acute
(2) curative
(3) short-term
(4) intermittent

d. systems

(1) inadequate security

(2) weak rule of law

(3) limited accountability framework

(4) uncoordinated humanitarian action

2. Project feasibility constraints
a. security
b. political
c. administrative
d. logistical
e. technical
f. economic
g. developmental

3. Hazard-specific constraints (complex emergencies)
a. hostile armed elements
b. limited access
c. overburdened provincial services
d. limited information on beneficiaries

V. SOCIETAL RESILIENCE (hazards + environment + infrastructure + institutions + livelihoods)

\section{A. Comprehensive Disaster Risk Management}

1. DRM/DRR (where risk = hazard $\mathrm{x}$ vulnerability; also likelihood $\mathrm{x}$ impact)

2. Hazard and vulnerability analysis

3. Hazard and structural mitigation (eg climate change adaptation plans)

4. Disaster plans
a. all-hazard plans vs hazard-specific plans
b. preparedness plans
c. contingency plans
d. evacuation plans
e. repurposing plans 
f. business continuity plans

g. recovery plans

h. R2D considerations

5. Early warning/early action systems

6. Emergency operation centers

7. Emergency relief supply systems

8. Disaster simulation exercises, tabletops, drills

\section{B. Political}

1. Proactive legislation and policy (not reactive)

2. Clear non-overlapping mandates

3. Funding commitment to comprehensive disaster management

4. Vertical and horizontal linkages in policy implementation

5. Comprehensive approaches to ecosystem protection rather than fragmentation based upon political jurisdictions

6. Generic approaches to managing technical (best) practices, but local approaches to managing community vulnerabilities

7. Communication and cooperation enabled between public and private sector, national level and local communities, and individual jurisdictions

\section{Socioeconomic}

1. Environmental impact of population growth

2. Environmental impact of dominant practices in land use and key livelihood sectors - agriculture, mining, manufacturing, pastoralism, etc.

3. Environmental and economic impact of hazard-specific zones, damages, and losses on biodiversity, ecosystems, and communities

4. Poverty alleviation and economic diversification programs

5. Inclusiveness thru use of gender disaggregated data

6. Indigenous knowledge and contribution to DRR

7. (Re)insurance mechanisms

\section{Institutional}

1. Leadership training programs

2. Transparent resource management

3. Open redundant communication channels

4. Streamlined work flows (no waste)

5. Robust information management and information sharing

6. MEAL programs

7. Accountability frameworks

8. Inter-agency coordination

9. Partnership development 


\section{E. Environment and Infrastructure}

1. Hazard zoning

2. Hazard and structural mitigation

3. Remote sensing and early warning systems

4. Preventive maintenance

\section{F. Community Capacities and Vulnerabilities}

1. Lifeline protection (key infrastructure assets and systems) NB food for assets (using food, cash, or vouchers) programs can help build physical assets listed below (see VA2)

a. security

b. communication

c. transportation

d. energy

e. environmental health (eg water supply, food security, shelter and housing)

f. public health (eg disease surveillance, primary prevention)

g. health

(1) safe hospitals program

h. education to achieve a literate and informed public

i. information and DRR technologies

2. Social protection and safety nets (focus on women, vulnerable groups)

3. Livelihood protection

\section{DEVELOPMENT PROGRAMS}

A. Strategic Objectives (USAID Policy Framework for Bilateral Foreign Aid [3])

1. Promote transformational development

Support far-reaching, fundamental changes in relatively stable developing countries, with emphasis on improvements in governance and institutions, human capacity, and economic structure, so that countries can sustain further economic and social progress without depending on foreign aid. Focus on those countries with significant need for assistance and with adequate (or better) commitment to ruling justly, promoting economic freedom, and investing in people.

2. Strengthen fragile states Reduce fragility and establish the foundation for development progress by supporting stabilization, reform, and capacity development in fragile states when and where U.S. assistance can make a significant difference.

3. Support strategic states

Help achieve major U.S. foreign policy goals in specific countries of high priority from a strategic standpoint. 
4. Provide humanitarian relief

Help meet immediate human needs, save lives, and alleviate suffering in countries afflicted by violent conflict, crisis, natural disasters, or persistent dire poverty.

5. Address global issues

HIV/AIDS, other infectious diseases, climate change, direct support for international trade agreements, and counter narcotics.

B. Approaches (USAID Gender Equality and Female Empowerment Policy [4])

1. Adopt inclusive approach

2. Integrate gender equality into all work

3. Build partnerships

4. Harness science, technology, and innovation

5. Address unique challenges in crises and conflict-affected communities

6. Serve as thought leader

7. Be accountable

C. Partnership Mechanisms (US National Strategy for Pandemic Influenza [5])

1. International cooperation to protect lives and health

2. Timely and sustained high-level political leadership to the disease

3. Transparency in reporting of cases of disease in humans and in animals caused by strains that have pandemic potential to increase understanding, enhance preparedness, and ensure rapid and timely response to potential outbreaks

4. Immediate sharing of epidemiological data and clinical samples with the World Health Organization (WHO) and the international community to characterize the nature and evolution of any outbreaks as quickly as possible

5. Prevention and containment of an incipient epidemic through capacity building and in-country collaboration with international partners

6. Rapid response to the first signs of accelerated disease transmission

7. Work in a manner supportive of key multilateral organizations (WHO, FAO, OIE)

8. Timely coordination of bilateral and multilateral resource allocations; dedication of domestic resources (human and financial); improvements in public awareness; and development of economic and trade contingency plans

9. Increased coordination and harmonization of preparedness, prevention, response and containment activities among nations

10. Actions based on the best available science 
D. Program Innovations at Community Level

1. Community interventions

a. sector-wide approaches (SWAps)

b. integrated health interventions
(1) $\mathrm{PHC}$
(2) $\mathrm{SPHC}$
(3) IMCI

c. home-based interventions

(1) CTC

d. microfinance

(1) Grameen Bank

2. Best practice program models (centers of excellence arising within a community)

a. Matlab Health Research Center (part of ICDDR, Bangladesh)

b. Shoklo Malaria Research Unit, Tak Province, Thailand

c. Fistula Hospital, Addis Ababa, Ethiopia

d. Behrhorst Clinic, Guatemala

3. Self-replicating centers of excellence

a. Fistula Hospital, Addis Ababa, Ethiopia

\section{SEEDS OF CHANGE}
A. Innovators
B. Thought Leaders
C. Change Agents and Influencers
D. Early Adopters
E. Craft Groups
F. Centers of Excellence
G. Communities of Practice
H. Global Alliance
I. Crowds

\section{References}

1. Inter-agency Standing Committee. Guidance Note on Using the Cluster Approach to Strengthen Humanitarian Response. November 24, 2006. Available from: https://interagencystandingcommittee.org/system/files/legacy_files/Cluster $\% 20$ implementation \%2C\%20Guidance\%20Note\%2C\%20WG66\%2C\%2020061115-.pdf.

2. OCHA. Global Cluster Leads (as of June 2012). Available from: http://reliefweb.int/ sites/reliefweb.int/files/resources/map_2809.pdf. 
3. USAID. Policy framework for bilateral foreign aid. January 2006. Available from USAID's Development Experience Clearinghouse https://www.dec.usaid.gov, and https://www.usaid.gov/sites/default/files/documents/1868/201mam.pdf.

4. USAID. Gender equality and female empowerment policy. March 2012. Available from: https://www.usaid.gov/sites/default/files/documents/1865/ GenderEqualityPolicy_0.pdf.

5. US Homeland Security Council. National strategy for pandemic influenza. November 2005. Available from: http://www.flu.gov/planning-preparedness/federal/ pandemic-influenza.pdf. 


\section{Document 7.2 SECURITY SECTOR}

\section{DEFINITIONS (Rome Statute [1])}

A. Crimes (within the International Criminal Court's Jurisdiction) (Article 5)

1. Genocide (Article 6) - acts committed with intent to destroy, in whole or in part, a national, ethnic, racial, or religious group

a. killing members of the group

b. causing serious bodily or mental harm to members of the group

c. inflicting on the group conditions of life calculated to bring about its physical destruction in whole or in part

d. imposing measures intended to prevent births within the group

e. forcibly transferring children of the group to another group

2. Crimes against humanity (Article 7) - acts committed as part of a widespread or systematic attack against any civilian population, with knowledge of the attack

a. murder

b. extermination

c. enslavement

d. deportation

e. imprisonment in violation of international law

f. torture

g. rape, sexual slavery, enforced prostitution, forced pregnancy, enforced sterilization, or other comparable form of sexual violence

h. persecution on political, racial, national, ethnic, cultural, religious, gender, or other grounds universally recognized as impermissible under international law

i. enforced disappearance

j. apartheid

$\mathrm{k}$. other inhumane acts intentionally causing great suffering or serious injury to body or to mental or physical health

3. War crimes (Article 8)

a. grave breaches of the Geneva Conventions of 12 Aug 1949

(1) willful killing

(2) torture or inhumane treatment including biological experiments

(3) willfully causing great suffering

(4) extensive destruction and appropriation of property

(5) compelling a POW to serve in the armed forces of a hostile power

(6) willfully depriving a POW of the right to a fair trial

(7) unlawful deportation

(8) taking of hostages 
b. serious violations of laws and customs applicable in international armed conflict

(1) intentionally directing attacks against the civilian population or against civilians not taking direct part in hostilities

(2) intentionally directing attacks against civilian objects

(3) intentionally directing attacks against personnel, installations, material, units, or vehicles involved in humanitarian assistance or peacekeeping mission

(4) intentionally launching an attack in the knowledge that it will cause incidental civilian loss of life or severe damage to the natural environment

(5) attacking undefended towns, villages, dwellings, or buildings which are not military targets

(6) killing or wounding a combatant who has surrendered

(7) improper use of a flag of truce, flag or insignia or uniform of the enemy or of the UN, or emblems of the Geneva conventions resulting in death or serious personal injury

(8) transfer by the Occupying Power of parts of its own civilian population into the territory it occupies, or the deportation or transfer of all or parts of the population of the occupied territory within or outside the territory

(9) intentionally directing attacks against buildings dedicated to religion, education, art, science, charitable purposes, historic monuments, hospitals, and places where sick are collected, provided they are not military objectives

(10) subjecting persons to physical mutilation or to medical or scientific experiments which are not justified by the medical treatment nor carried out in his/her interest

(11) killing or wounding treacherously individuals belonging to the hostile nation or army

(12) declaring that no quarter will be given

(13) destroying or seizing the enemy's property unless such be imperatively demanded by the necessities of war

(14) declaring abolished, suspended, or inadmissible in a court of law the rights and actions of the nationals of the hostile party

(15) compelling the nationals of the hostile party to take part in the operations of war directed against their own country

(16) pillaging a town or place, even when taken by assault

(17) employing poison or poison weapons

(18) employing asphyxiating, poisonous or other gases, and all analogous liquids, materials, or devices 
(19) employing bullets which expand or flatten easily in the human body

(20) employing weapons, projectiles, material and methods of warfare which cause superfluous injury or unnecessary suffering

(21) committing outrages upon personal dignity, in particular humiliating and degrading treatment

(22) committing rape, sexual slavery, enforced prostitution, forced pregnancy, enforced sterilization, or other comparable form of sexual violence

(23) utilizing a civilian or other protected person to render certain areas or military forces immune from military operations

(24) intentionally directing attacks against buildings, material, medical units, transport, and personnel using the emblems of the Geneva Conventions in conformity with international law

(25) conscripting or enlisting children under the age of 15 years

c. serious violations of common article 3 applicable in noninternational armed conflict, ie acts vs persons taking no active part in the hostilities, including armed forces placed hors de combat by sickness, wounds, detention, or other cause (1) violence to life and person

(2) outrages upon personal dignity

(3) taking of hostages

(4) passing of sentences and carrying out of executions

d. non-applicability of $\mathrm{c}$ to internal disturbances (riots, sporadic violence, etc)

e. other serious violations of laws and customs applicable in non-international armed conflict (gen // 3b above)

4. Crime of aggression (provision to be adopted)

\section{ARMED PERPETRATORS}
A. Regular Military (enlisted or conscripted)
B. Militias
C. Mercenaries
D. Ethnic and Religious Extremists
E. Affiliates of A-D
F. Rogue Elements and Dissidents
G. Peacekeeping Forces
H. Organized Criminals
I. Petty Criminals

\section{HEALTH CONSEQUENCES OF SECURITY BREAKDOWN}

\section{A. Decreased Access and Protection for Beneficiaries}

1. Besieged areas

2. Lawlessness 
3. Forced displacement

4. Decreased access to lifeline systems (water, sanitation, food, power, communications) thru destruction, roadblocks, curfews, discrimination

5. Decreased access to humanitarian assistance as above

6. Discrimination and persecution
a. age
b. gender
c. race
d. religion
e. ethnicity
f. geographic origin
g. political affiliation
h. socioeconomic status
i. sexual orientation
j. HIV status
k. disability status
1. migratory status

\section{B. Increased Morbidity and Mortality}

1. Direct trauma-death, injury, disability, murder, mutilations, torture, SGBV, psychosocial suffering

2. Diseases of overcrowding and displacement

3. Outbreaks of preventable and previously controlled diseases

4. Untreated chronic illnesses

5. Morbidity \& mortality multipliers (measles, malnutrition, TB/ HIV)

6. High risk behavior to address basic needs
a. exposure to military conflict and ordnance
b. criminality
c. transactional/survival sex
d. child exploitation

\section{Degradation of Health System}

1. Damage/destruction of health facilities and critical infrastructure

2. Degradation/disruption of health services complicated by increased demand from population influxes and decreased access to care

3. Threats to, attacks on, and attrition of health personnel

4. Disruption of medical logistics

5. Disruption of health information systems and telecommunications at large

6. Under-resourced health financing

7. Weak health governance

\section{Loss of Human Capital and Physical Assets \\ E. Loss of Development Opportunity for Society}




\section{SECURITY STEPS TO INITIATING SERVICES IN CONFLICT ZONES}

A. Identification of Ground Contacts and Formation of Security Network

B. Initiation of UNHAS Flights

C. Initiation of Day Missions

1. security detail on team with comms equipment and plan

2. contingency packs with tent, sleeping bags, and water in case of security breakdown

3. ground situation to be ascertained before mission departure

D. Establishment of Humanitarian Hub

\section{SECURITY OBJECTIVES}

A. Unimpeded Access to Beneficiaries

1. Registration/verification

2. Protection

3. Humanitarian assistance

4. Family reunification

5. Information dissemination

B. Security for Refugees and Humanitarian Personnel

1. MOSS and SOPs

2. Security Focal Points

3. Security Ops Center with daily briefings

4. Cessation of hostilities

5. DDRRR

6. Peace agreements

\section{Law Enforcement}

1. Appropriate civil authorities

2. Search for accountability

3. Perpetrators of violence are arrested, charged, and tried in a civil court of law

D. Free and Informed Choices

E. Program Participation of Beneficiaries (especially women)

F. Relocation (only if voluntary, people are medically fit, and families are united)

\section{Reference}

1. Rome Statute of the International Criminal Court, July 17, 1998, 2187 U.N.T.S. 90. Available from: http://untreaty.un.org/cod/icc/statute/romefra.htm. 


\section{Document 7.3 HEALTH SECTOR}

\section{Glossary}

$\begin{array}{ll}\text { ABX } & \text { antibiotics } \\ \text { AFP } & \text { acute flaccid paralysis } \\ \text { AIDS } & \text { acquired immune deficiency syndrome } \\ \text { ASDR } & \text { age-specific death rate } \\ \text { BCG } & \text { bacillus Calmette-Guérin } \\ \text { BMI } & \text { body mass index } \\ \text { c } & \text { with } \\ \text { cc } & \text { cubic centimeter } \\ \text { CDR } & \text { crude death rate } \\ \text { CFR } & \text { case fatality ratio } \\ \text { CI } & \text { confidence interval } \\ \text { cm } & \text { centimeter } \\ \text { CMAM } & \text { community-based management of acute malnutrition } \\ \text { CSS } & \text { cluster sample survey } \\ \text { CTC } & \text { community therapeutic care (malnutrition management) } \\ \text { d } & \text { day } \\ \text { D5 } & \text { dextrose 5\% in water } \\ \text { D10 } & \text { dextrose } 10 \% \text { in water } \\ \text { D\&C } & \text { dilation and curettage } \\ \text { D } & \text { design effect } \\ \text { DTP } & \text { diphtheria + tetanus + pertussis vaccine } \\ \text { DRR } & \text { disaster risk reduction } \\ \text { EOC } & \text { Emergency Operations Center } \\ \text { EPI } & \text { Expanded Programme on Immunization } \\ \text { gm } & \text { gram } \\ \text { GAM } & \text { global acute malnutrition } \\ \text { gtt } & \text { drops } \\ \text { h } & \text { hour } \\ \text { H } & \text { height } \\ \text { HAM } & \text { height-for-age median } \\ \text { HAZ } & \text { height-for-age z score } \\ \text { HB } & \text { hepatitis B } \\ \text { HCW } & \text { health care worker } \\ \text { HH } & \text { household } \\ \text { Hib } & \text { Haemophilus influenzae type b } \\ \text { HIV } & \text { human immunodeficiency virus } \\ \text { ICDDR } & \text { International Centre for Diarrhoeal Disease Research, } \\ \text { ID } & \text { Bangladesh } \\ \text { incident command system } & \text { infectious disease } \\ & \text { Integrated Management of Childhood Illness (WHO initive } \\ & \end{array}$


IMPAC Integrated Management of Pregnancy and Childbirth (WHO initiative)

IMR infant mortality rate

IPD inpatient department

IV intravenous

kg kilogram

L liter

L\&D labor and delivery

m meter

MAM moderate acute malnutrition

MCI mass casualty incident

MCV measles-containing vaccine

MD medical doctor

mg milligram

MISP Minimum Initial Service Package (used in reproductive health)

$\mathrm{mL} \quad$ milliliter

MMR measles + mumps + rubella vaccine

mo month

MR measles + rubella vaccine

MSF Médecins sans Frontières

MUAC mid-upper arm circumference

NGO non-governmental organization

NGT nasogastric tube

NID national immunization day(s)

NL normal

NS normal saline

O10 over 10 year-old

OPD outpatient department

OPV oral polio vaccine

OR operating room

ORS oral rehydration solution

OTP outpatient therapeutic program

PCV pneumococcal conjugate vaccine

PLW pregnant and lactating women

PO by mouth

ppm parts per million

RL Ringer's lactate

RUSF ready-to-use supplementary food

RUTF ready-to-use therapeutic food

$\mathrm{RV}$ rotavirus

$\mathrm{Rx} \quad$ medication

SAM severe acute malnutrition

SC stabilization center

SD standard deviation

SFC supplementary feeding center

SFP supplementary feeding program 


$\begin{array}{ll}\text { SIA } & \text { supplemental immunization activities } \\ \text { SOP } & \text { standard operating procedure } \\ \mathrm{t} & \text { teaspoon } \\ \mathrm{T} & \text { tablespoon } \\ \text { TB } & \text { tuberculosis } \\ \text { TFC } & \text { therapeutic feeding center } \\ \text { Tx } & \text { treatment } \\ \text { U5 } & \text { under 5 year-old } \\ \text { U5DR } & \text { under 5 year-old death rate } \\ \text { U5MR } & \text { under 5 year-old mortality rate } \\ \text { U10 } & \text { under 10 year-old } \\ \text { W } & \text { weight } \\ \text { WASH } & \text { water, sanitation, and hygiene } \\ \text { W/H } & \text { weight-for-height } \\ \text { WHM } & \text { weight-for-height median } \\ \text { WHO } & \text { World Health Organization } \\ \text { WHZ } & \text { weight-for-height z score } \\ \text { wk } & \text { week } \\ \text { YF } & \text { yellow fever } \\ \text { yr } & \text { year }\end{array}$




\section{CORE DISCIPLINES IN DISASTER HEALTH}

\section{A. Clinical Medicine}

1. Prehospital care

2. Primary, preventive, and basic services

a. comprehensive primary health care

b. child health services

c. reproductive health services

d. trauma services

e. mental health services

f. chronic diseases

g. infection control

3. Referral care

\section{B. Public Health}

1. Rapid epidemiological assessment (minimum essential data sets)

2. Inter-agency health coordination

3. Standardized case management

4. Environmental health (minimum standards)

5. Epidemic preparedness and response

6. Disease surveillance (surveillance case definitions, data flow, and analysis)

7. Special surveys (cluster sample surveys)

8. Health policy and personnel planning

9. Immunization programs (EPI)

10. Medical logistics

\section{Disaster Management}

1. Site security

2. Urban search and rescue

3. Hazard-specific issues (hazard analysis, mitigation, decontamination, vulnerability reduction)

4. Disaster response modalities (ICS/EOC)

5. Geographic information systems

6. Public information

7. Community recovery

\section{WHO Mandated Responsibilities in Disaster}

1. Core functions (World Health Assembly Res 58.1)

a. needs assessment

b. health coordination

c. gap filling

d. capacity building

NB protection \& nutrition are beyond the mandate of WHO 
2. Critical functions in emergency response (WHO Emergency Response Framework)
a. leadership
b. information
c. technical expertise
d. core services

\section{PRIMARY HEALTH CARE PROGRAMS}

A. Primary Health Care (Declaration of Alma Ata [1])
1. Health education
2. Food and nutrition
3. Water and sanitation
4. Maternal and child health, family planning
5. Immunization
6. Prevention of endemic disease
7. Treatment of common disease and injury
8. Essential drugs

B. Selective Primary Health Care (Walsh and Warren [2])

1. Growth monitoring

2. Oral rehydration therapy

3. Breast feeding

4. Immunization

5. Female literacy

6. Family planning

7. Food supplements

\section{DISEASE PREVENTION}

\section{A. Definitions}

1. Primary (individual without disease)

Goal-prevent the disease from starting (consider host-agentenvironment)
a. limit pathogenesis of a disease-causing agent (eg lower cholesterol levels)
b. alter the environment to keep the disease from humans (eg regulate asbestos exposures)
c. strengthen host resistance to the disease (eg measles immunization, water fluoridation)

2. Secondary (individual with disease but without symptoms)

Goal-prevent disease symptoms and complications from developing
a. intervene after exposure (eg rabies vaccination)
b. detect and treat disease before it becomes symptomatic (eg cancer screening)


c. prevent disease spread by treating contacts (eg TB exposure treatment)

3. Tertiary (individual with symptomatic disease)

Goal-cure or control of clinical disease (bulk of clinical medicine)

a. cure disease or reverse clinical manifestations (eg appendectomy, thrombolysis)

b. control disease progression to avoid complications (eg retroviral drugs, anticoagulation in atrial fibrillation)

c. control spread of disease to others (eg TB case management)

\section{B. Vaccinations}

1. Infant schedule (WHO)

Table 7.3.1

Infant Vaccination Schedule

\begin{tabular}{|l|l|}
\hline \multicolumn{1}{|c|}{ Age } & \multicolumn{1}{c|}{ Vaccine } \\
\hline Birth & BCG, HB-1, \pm OPV birth dose \\
\hline 6 wks & DTP-1, OPV/IPV-1, HB-2, Hib-1, PCV-1, RV-1 \\
\hline 10 wks & DTP-2, OPV/IPV-2, HB-3, Hib- 2, PCV-2, RV-2 \\
\hline 14 wks & DTP-3, OPV/IPV-3, HB-4, Hib-3, PCV-3, RV-3 \\
\hline $9-12$ mo & MCV-1 (eg MR or MMR) \\
\hline 15 mo & MCV-2 \\
\hline
\end{tabular}

NB Table does not convey all options for vaccine administration. Diphtheria toxoid is not manufactured as monovalent vaccine. It is combined with tetanus toxoid-either full-strength dose for pediatric use (DT), or reducedstrength dose for adult use (Td). These are combined with acellular or whole-cell pertussis antigens - either full-strength dose for pediatric use (DTaP, DTwP), or reduced-strength dose for adolescent/adult use (Tdap).

DTP-1 is a good indicator of access to care.

DTP-3 is a good indicator of ultimate service delivery.

2. Immunization program 
Table 7.3.2

Immunization Program Components

\begin{tabular}{|l|l|l|}
\hline \multicolumn{1}{|c|}{ Intervention } & \multicolumn{1}{c|}{ Polio } & \multicolumn{1}{c|}{ Measles } \\
\hline Routine immunization & + & + \\
\hline $\begin{array}{l}\text { Supplementary } \\
\text { immunization }\end{array}$ & $\begin{array}{l}\text { National immunization } \\
\text { days (NID) }\end{array}$ & $\begin{array}{l}\text { Supplementary } \\
\text { immunization activities } \\
\text { (SIA) }\end{array}$ \\
\hline Surveillance & $\begin{array}{l}\text { Acute flaccid paralysis } \\
\text { (AFP) }\end{array}$ & Case-based \\
\hline Case follow-up & Mop-up campaign & Case management \\
\hline
\end{tabular}

3. Vaccine quantities

Table 7.3.3

Calculation of Vaccine Quantities

\begin{tabular}{|l|r|}
\hline \multicolumn{1}{|c|}{ Indicator \& benchmark } & Quantity \\
\hline Total population & 100,000 \\
\hline Target population $(6 \mathrm{mo}-15 \mathrm{yr}) 45 \%$ total & 45,000 \\
\hline Cover objective $=100 \%$ & 45,000 \\
\hline Number of doses (1 for measles) & 45,000 \\
\hline Expected loss 15\% & 52,940 \\
\hline Increase reserve by 25\% & 66,175 \\
\hline Round up & 67,000 \\
\hline
\end{tabular}

\section{CLINICAL FACILITIES}

\section{A. Generic Profile}

1. Quantity

$\begin{array}{ccc}\text { Fixed } & \text { Health } & \text { District } \\ \text { Clinics } & \text { Centers } & \text { Hosp }\end{array}$

2. Catchment population $\quad 5-10 \mathrm{k}<50 \mathrm{k} \quad 100 \mathrm{k}$

3. Access to facility self self referral

4. Staffing
a. quantity $(\sim 1 / 1000$ p)
2-5 staff
5-10 staff
variable
b. skill mix
$1 \mathrm{HCW} 5 \mathrm{HCW}, 1 \mathrm{MD}$
$5 \mathrm{MD}, 1$
surgeon

5. Caseload (\% of catchment

$$
\text { population/d) } \quad 1-10 \% \quad 1-10 \%
$$

6. Bed capacity $(10 / 10,000$ population $)$

7. Bed occupancy

8. Referral load (\% of caseload) $10 \% \quad 10 \%$

9. Referral system

10. Clinical services OPD, ORS + IPD, L\&D + OR 
a. departments/units (verify functioning of expected services)

(1) clinical
(a) OPD
(b) IPD
(c) L\&D
(d) surgery
(e) other

(2) dispensary

(3) diagnostic lab

(4) blood bank

(5) radiology

\section{REPRODUCTIVE HEALTH}

\section{A. Safe Motherhood (IMPAC, MISP)}

NB $15 \%$ of pregnant women have complications requiring emergency OB care; $5 \%$ (3-7\%) need C-section; $10 \%$ of deliveries will have primary post-partum hemorrhage within $24 \mathrm{~h}$

1. Causes of maternal death
a. other emergencies $22 \%$
b. hemorrhage $18 \%$
c. eclampsia $12 \%$
d. obstructed labor $8 \%$ ruptured uterus $-1 \mathrm{~d}$
e. sepsis $9 \%$ ante-partum $-12 \mathrm{~h}$, post-partum $-2 \mathrm{~h}$
f. unsafe abortion $18 \%$ $2 \mathrm{~d}$
$3 \mathrm{~d}$
$6 \mathrm{~d}$
g. other indirect (malaria, HIV) 13\% variable

Time to death

2. Basic emergency obstetric care (1 facility/125 k pop)
a. parenteral antibiotics
b. parenteral oxytocics
c. parenteral anticonvulsants for pre-eclampsia \& eclampsia
d. manual removal of placenta
e. removal of retained products (vacuum aspiration, D\&C)
f. assisted vaginal delivery (vacuum, forceps)

3. Comprehensive emergency obstetric care (as above plus below) (1 facility/500 k pop)
a. Caesarean section (5-15\% of deliveries)
b. blood transfusion

4. Treatment of obstructed labor

a. McRoberts maneuver-flex thighs at the hips and push knees to maternal abdomen

b. pressure on suprapubic area

c. proctoepisiotomy

d. Wood's corkscrew maneuver - rotate shoulders $180^{\circ}$ or to an oblique diameter to disengage the impacted shoulder

e. delivery of the posterior arm (> incidence of humerus and clavicle fractures) 
f. symphysiotomy

g. Zavanelli maneuver-replace head into the vagina and perform C-section

5. Essential newborn care

a. basic newborn resuscitation

b. warmth (drying and kangaroo care)

c. eye prophylaxis (tetracycline ointment)

d. clean cord care

e. early and exclusive breast feeding

6. Delays to care

a. delay in deciding to seek care

b. delay in reaching care

c. delay in receiving adequate care

VI. WATER AND SANITATION

A. Classification (D Mara, R Feachem [3])

Table 7.3.4

Environmental Classification of Water \& Excreta Related Diseases

\begin{tabular}{|c|c|c|}
\hline Diseases & Pathogen/disease & Preventive Measures \\
\hline \multirow[t]{4}{*}{$\begin{array}{l}\text { Fecal-oral = water- } \\
\text { borne + water-washed }\end{array}$} & $\begin{array}{l}\text { Virus - hepatitis } \\
\text { A/E, polio, rotavirus, } \\
\text { adenovirus }\end{array}$ & \multirow[t]{4}{*}{$\begin{array}{l}\text { Water-washed: }>\mathrm{H}_{2} \mathrm{O} \\
\text { quantity } \\
\text { Water-borne: }>\mathrm{H}_{2} \mathrm{O} \text { quality }\end{array}$} \\
\hline & $\begin{array}{l}\text { Bacteria- } \\
\text { Campylobacter, E. coli, } \\
\text { Salmonella, Shigella, } \\
\text { Vibrio }\end{array}$ & \\
\hline & $\begin{array}{l}\text { Protozoa-Entamoeba, } \\
\text { Balantidium, } \\
\text { Cryptosporidium, } \\
\text { Giardia, Isospora }\end{array}$ & \\
\hline & $\begin{array}{l}\text { Helminths - Ascaris, } \\
\text { Enterobius }\end{array}$ & \\
\hline \multirow[t]{3}{*}{$\begin{array}{l}\text { Non-fecal-oral water- } \\
\text { washed }\end{array}$} & $\begin{array}{l}\text { Skin infections- } \\
\text { scabies, leprosy, yaws }\end{array}$ & \multirow[t]{3}{*}{$\begin{array}{l}>\mathrm{H}_{2} \mathrm{O} \text { quantity; } \\
>\text { Hygiene education }\end{array}$} \\
\hline & $\begin{array}{l}\text { Eye infections - } \\
\text { trachoma, conjunctivitis }\end{array}$ & \\
\hline & Louse-borne fevers & \\
\hline
\end{tabular}


Document 7.3 167

\begin{tabular}{|c|c|c|}
\hline Diseases & Pathogen/disease & Preventive Measures \\
\hline \multirow[t]{2}{*}{ Water-based } & $\begin{array}{l}\text { Bacteria-Leptospira, } \\
\text { Francisella, Legionella }\end{array}$ & \multirow[t]{2}{*}{$\begin{array}{l}<\text { Contact with } \\
\text { contaminated } \mathrm{H}_{2} \mathrm{O}\end{array}$} \\
\hline & $\begin{array}{l}\text { Helminths- } \\
\text { Schistosoma, } \\
\text { Clonorchis, Fasciola, } \\
\text { Paragonimus, } \\
\text { Dracunculus }\end{array}$ & \\
\hline $\begin{array}{l}\text { Insect-vectored, water- } \\
\text { related } \\
\text { Mosquitoes (breeding } \\
\text { in water), flies (biting/ } \\
\text { breeding near water) }\end{array}$ & $\begin{array}{l}\text { Malaria, dengue, Rift } \\
\text { Valley fever, Japanese } \\
\text { encephalitis, yellow } \\
\text { fever, sleeping sickness, } \\
\text { oncho, filariasis }\end{array}$ & $\begin{array}{l}\text { Destroy breeding sites; } \\
\text { Use barrier precautions } \\
\text { (ITNs) }\end{array}$ \\
\hline $\begin{array}{l}\text { Insect-vectored, } \\
\text { excreta-related } \\
\text { Flies, cockroaches }\end{array}$ & $\begin{array}{l}\text { Includes all fecal-oral } \\
\text { diseases + water- } \\
\text { based helminths } \\
+ \text { geohelminths + } \\
\text { taeniasis }\end{array}$ & > Environmental hygiene \\
\hline Rodent-vectored & $\begin{array}{l}\text { Similar to insect- } \\
\text { vectored, excreta- } \\
\text { related but includes } \\
\text { all water-based } \\
\text { pathogens listed (except } \\
\text { Legionella) }\end{array}$ & $\begin{array}{l}>\text { Rodent control; } \\
<\text { Contact with } \\
\text { contaminated } \mathrm{H}_{2} \mathrm{O}\end{array}$ \\
\hline Geohelminths & $\begin{array}{l}\text { Roundworm, } \\
\text { hookworm, whipworm }\end{array}$ & Excreta disposal \\
\hline Taenia & $\begin{array}{l}\text { Beef and pork } \\
\text { tapeworm }\end{array}$ & $\begin{array}{l}\text { Excreta disposal; } \\
\text { Proper meat cooking }\end{array}$ \\
\hline
\end{tabular}

Source: D Mara, R Feachem in Journal of Environmental Engineering (C) 1999. Used with permission of ASCE. 
B. Prevention (adapted from R Feachem [4])

Table 7.3.5

Water \& Excreta Related Disease Prevention Measures

\begin{tabular}{|c|c|c|c|c|c|c|c|}
\hline \multirow[t]{2}{*}{ Disease } & \multicolumn{7}{|c|}{ Prevention Measures } \\
\hline & $\begin{array}{c}\mathrm{H}_{2} \mathrm{O} \\
\text { quality }\end{array}$ & $\begin{array}{c}\mathrm{H}_{2} \mathrm{O} \\
\text { quantity }\end{array}$ & $\begin{array}{l}\text { Excreta } \\
\text { disposal }\end{array}$ & $\begin{array}{c}\text { Excreta } \\
\mathbf{T x}\end{array}$ & $\begin{array}{l}\text { Personal } \\
\text { hygiene }\end{array}$ & $\begin{array}{c}\text { Waste } \\
\mathrm{H}_{2} \mathrm{O} \\
\text { disposal }\end{array}$ & $\begin{array}{c}\text { Food } \\
\text { hygiene }\end{array}$ \\
\hline \multicolumn{8}{|l|}{$\begin{array}{l}\text { Diarrheal } \\
\text { disease } \\
\text { \& enteric } \\
\text { fevers }\end{array}$} \\
\hline Viral & 2 & 2 & 1 & 1 & 2 & 0 & 1 \\
\hline Bacterial & 3 & 3 & 2 & 1 & 3 & 0 & 3 \\
\hline Protozoal & 1 & 3 & 2 & 1 & 3 & 0 & 2 \\
\hline $\begin{array}{l}\text { Polio, } \\
\text { Hepatitis A }\end{array}$ & 1 & 3 & 2 & 1 & 3 & 0 & 1 \\
\hline $\begin{array}{l}\text { Water- } \\
\text { washed } \\
\text { (skin, eye, } \\
\text { louse-borne } \\
\text { disease) }\end{array}$ & 0 & 3 & 0 & 0 & 3 & 0 & 0 \\
\hline \multicolumn{8}{|l|}{ Water-based } \\
\hline Schisto & 1 & 1 & 3 & 2 & 1 & 0 & 0 \\
\hline $\begin{array}{l}\text { Guinea } \\
\text { worm }\end{array}$ & 3 & 0 & 0 & 0 & 0 & 0 & 0 \\
\hline Clonorchis & 0 & 0 & 2 & 2 & 0 & 0 & 3 \\
\hline \multicolumn{8}{|l|}{$\begin{array}{l}\text { Water- } \\
\text { related }\end{array}$} \\
\hline Malaria & 0 & 0 & 0 & 0 & 0 & 1 & 0 \\
\hline Dengue, YF & 0 & 0 & 0 & 0 & 0 & 1 & 0 \\
\hline Filariasis & 0 & 0 & 3 & 0 & 0 & 3 & 0 \\
\hline $\begin{array}{l}\text { Worms } \\
\text { without } \\
\text { intermediate } \\
\text { host }\end{array}$ & 0 & 1 & 3 & 2 & 1 & $0-1$ & $1-2$ \\
\hline $\begin{array}{l}\text { Worms with } \\
\text { intermediate } \\
\text { host }\end{array}$ & 0 & 0 & 3 & 3 & 0 & 0 & 3 \\
\hline
\end{tabular}

Source: R Feachem in Water Supply and Sanitation in Developing Countries () 1983. Used with permission of Chartered Institution of Water and Environmental Management. $0=$ no importance 3 = great importance 
A range of generic prevention measures should be considered for its impact on diseases in a biological "all-hazards" environment. Overall, excreta disposal, water quantity, personal hygiene, and food hygiene commonly contribute more to environmental health than do other listed measures. Epidemic threats will oblige heightened consideration of disease-specific strategies for prevention and control.

C. Water Treatment (bold text of particular relevance in clinical facilities)

$$
\begin{aligned}
1 \mathrm{ppm}= & 1 \mathrm{mg} / \mathrm{kg} \text { (solids) } \\
= & 1 \mathrm{mg} / \mathrm{L} \text { (liquids) }=1 \mathrm{ug} / \mathrm{mL} \text { (liquids) = basic unit of } \\
& \text { measure for chloroscopes } \\
& : 10,000 \mathrm{ppm}=1 \%
\end{aligned}
$$

\begin{tabular}{|c|c|c|c|}
\hline PPM & Equivalents & Concentration \% & Use \\
\hline 0.5 & $\begin{array}{l}0.5 \mathrm{mg} / \mathrm{L} \\
0.0005 \mathrm{gm} / \mathrm{L}, \mathrm{mg} / \mathrm{mL} \\
0.00005 \mathrm{gm} / 100 \mathrm{~mL}\end{array}$ & $0.00005 \%$ & $\begin{array}{l}\text { Piped water systems, goal for } \\
\text { all end user drinking water }\end{array}$ \\
\hline 1 & $\begin{array}{l}1 \mathrm{mg} / \mathrm{L} \\
0.001 \mathrm{gm} / \mathrm{L}, \mathrm{mg} / \mathrm{mL} \\
0.0001 \mathrm{gm} / 100 \mathrm{~mL}\end{array}$ & $0.0001 \%$ & Standpost systems \\
\hline 2 & $\begin{array}{l}2 \mathrm{mg} / \mathrm{L} \\
0.002 \mathrm{gm} / \mathrm{L}, \mathrm{mg} / \mathrm{mL} \\
0.0002 \mathrm{gm} / 100 \mathrm{~mL}\end{array}$ & $0.0002 \%$ & $\begin{array}{l}\text { Pre-treatment for selected/ } \\
\text { known water sources - tanker } \\
\text { trucks at filling stations, } \\
\text { protected tube wells, clear } \\
\text { rainwater }\end{array}$ \\
\hline 5 & $\begin{array}{l}5 \mathrm{mg} / \mathrm{L} \\
0.005 \mathrm{gm} / \mathrm{L}, \mathrm{mg} / \mathrm{mL} \\
0.0005 \mathrm{gm} / 100 \mathrm{~mL}\end{array}$ & $0.0005 \%$ & $\begin{array}{l}\text { Pre-treatment for untested/ } \\
\text { unknown water sources- } \\
\text { unprotected wells, cloudy } \\
\text { surface water; filter/flocculate } \\
\text { before chlorinating }\end{array}$ \\
\hline 10 & $\begin{array}{l}10 \mathrm{mg} / \mathrm{L} \\
0.01 \mathrm{gm} / \mathrm{L}, \mathrm{mg} / \\
\mathrm{mL} \\
0.001 \\
\mathrm{gm} / 100 \mathrm{~mL}\end{array}$ & $0.001 \%$ & $\begin{array}{l}\text { Water with known fecal } \\
\text { contamination; filter/flocculate } \\
\text { before chlorinating }\end{array}$ \\
\hline 30 & $\begin{array}{l}30 \mathrm{mg} / \mathrm{L} \\
.03 \mathrm{gm} / \mathrm{L}, \mathrm{mg} / \mathrm{mL} \\
.003 \mathrm{gm} / 100 \mathrm{~mL}\end{array}$ & $0.003 \%$ & $\begin{array}{l}\text { Water from contaminated } \\
\text { well or borehole (needs } \\
\text { super-chlorination to achieve } \\
\text { breakpoint chlorination) }\end{array}$ \\
\hline 50 & $\begin{array}{l}50 \mathrm{mg} / \mathrm{L} \\
0.05 \mathrm{gm} / \mathrm{L}, \mathrm{mg} / \\
\mathrm{mL} \\
0.005 \\
\mathrm{gm} / 100 \mathrm{~mL}\end{array}$ & $0.005 \%$ & $\begin{array}{l}\text { Utensil rinse in restaurants } \\
\text { (USA) }\end{array}$ \\
\hline
\end{tabular}

Table 7.3.6

Chlorine Concentration and Uses 


\begin{tabular}{|c|c|c|c|}
\hline PPM & Equivalents & Concentration \% & Use \\
\hline 500 & $\begin{array}{l}500 \mathrm{mg} / \mathrm{L} \\
0.5 \mathrm{gm} / \mathrm{L}, \mathrm{mg} / \mathrm{mL} \\
0.05 \mathrm{gm} / 100 \mathrm{~mL}\end{array}$ & $0.05 \%$ & Hands, skin (eg wash points) \\
\hline 2000 & $\begin{array}{l}2000 \mathrm{mg} / \mathrm{L} \\
2 \mathrm{gm} / \mathrm{L}, \mathrm{mg} / \mathrm{mL} \\
0.2 \mathrm{gm} / 100 \mathrm{~mL}\end{array}$ & $\begin{array}{l}0.2 \% \\
(0.5 \% \text { at ICDDR) }\end{array}$ & $\begin{array}{l}\text { Cholera cots, covers, floors, } \\
\text { walls, equipment, clothing, } \\
\text { isolation areas (cholera, } \\
\text { dysentery, influenza epidemics) }\end{array}$ \\
\hline 10,000 & $\begin{array}{l}10,000 \mathrm{mg} / \mathrm{L} \\
10 \mathrm{gm} / \mathrm{L}, \mathrm{mg} / \mathrm{mL} \\
1 \mathrm{gm} / 100 \mathrm{~mL}\end{array}$ & $1 \%$ & Latrine door handles \\
\hline 20,000 & $\begin{array}{l}20,000 \mathrm{mg} / \mathrm{L} \\
20 \mathrm{gm} / \mathrm{L}, \mathrm{mg} / \mathrm{mL} \\
2 \mathrm{gm} / 100 \mathrm{~mL}\end{array}$ & $2 \%$ & $\begin{array}{l}\text { Body fluids (stool, vomitus)- } \\
\text { stool buckets, corpses } \\
\text { (disinfection for burial), shoes } \\
\text { (perimeter control) }\end{array}$ \\
\hline 50,000 & $\begin{array}{l}50,000 \mathrm{mg} / \mathrm{L} \\
50 \mathrm{gm} / \mathrm{L}, \mathrm{mg} / \mathrm{mL} \\
5 \mathrm{gm} / 100 \mathrm{~mL}\end{array}$ & $5 \%$ & Household bleach \\
\hline
\end{tabular}

Table 7.3.7

Chlorine Products and Dilution

\begin{tabular}{|l|l|l|l|l|}
\hline \multicolumn{1}{|c|}{ Product } & $\begin{array}{c}\text { Potable water } \\
\text { treatment } \\
\mathbf{0 . 0 0 0 5} \%\end{array}$ & $\begin{array}{l}\text { Hands and } \\
\text { skin cleaning } \\
\mathbf{0 . 0 5} \%\end{array}$ & $\begin{array}{c}\text { Bedding/ } \\
\text { clothes cleaning } \\
\mathbf{0 . 5} \%\end{array}$ & $\begin{array}{l}\text { Body fluid/ } \\
\text { corpse cleaning } \\
\mathbf{2 . 0} \%\end{array}$ \\
\hline $\begin{array}{l}\text { Household } \\
\text { bleach } \\
\text { Sodium } \\
\text { hypochlorite } \\
\text { solution } \\
5 \% \text { active } \\
\text { chlorine } \\
(50 \mathrm{mg} / \mathrm{mL})\end{array}$ & $\begin{array}{l}2.5 \mathrm{~mL} \text { in } \\
5 \mathrm{gal} \approx 8 \mathrm{gtt} / \mathrm{gal}, \\
2 \mathrm{gtt} / \mathrm{L} \approx 6 \mathrm{mg} / \mathrm{L} \\
\text { or } 6 \mathrm{PPM} \\
\text { NB double if } \\
\text { source is } \\
\text { contaminated }\end{array}$ & $\begin{array}{l}100 \mathrm{~mL} \text { in } \\
10 \mathrm{~L} \text { water }\end{array}$ & $\begin{array}{l}1 \text { liter in } \\
10 \mathrm{~L} \text { water }\end{array}$ & $\begin{array}{l}4 \text { liters in } 6 \mathrm{~L} \\
\text { water }\end{array}$ \\
\hline $\begin{array}{l}\text { Bleaching } \\
\text { powder } \\
\text { Chlorinated } \\
\text { lime } \\
30 \% \text { active } \\
\text { chlorine }\end{array}$ & & $\begin{array}{l}16 \mathrm{~g}(1 \mathrm{~T}) \\
\text { to } 10 \mathrm{~L} \\
\text { water }\end{array}$ & $\begin{array}{l}16 \mathrm{~g}(1 \mathrm{~T}) \\
\text { to } 1 \mathrm{~L}\end{array}$ & $64 \mathrm{~g}(4 \mathrm{~T})$ to $1 \mathrm{~L}$ \\
water & water \\
\hline
\end{tabular}




\begin{tabular}{|c|c|c|c|c|}
\hline Product & $\begin{array}{c}\text { Potable water } \\
\text { treatment } \\
\mathbf{0 . 0 0 0 5} \%\end{array}$ & $\begin{array}{c}\text { Hands and } \\
\text { skin cleaning } \\
0.05 \%\end{array}$ & \begin{tabular}{|c|} 
Bedding/ \\
clothes cleaning \\
$0.5 \%$
\end{tabular} & $\begin{array}{c}\text { Body fluid/ } \\
\text { corpse cleaning } \\
2.0 \%\end{array}$ \\
\hline $\begin{array}{l}\text { High-test } \\
\text { hypochlorite } \\
\text { (HTH) } \\
\text { Calcium } \\
\text { hypochlorite } \\
\text { Chlorine } \\
\text { granules } \\
70 \% \text { active } \\
\text { chlorine }\end{array}$ & & $\begin{array}{l}7 \mathrm{~g}(0.5 \mathrm{~T}) \\
\text { to } 10 \mathrm{~L} \\
\text { water }\end{array}$ & $\begin{array}{l}7 \mathrm{~g}(0.5 \mathrm{~T}) \\
\text { to } 1 \mathrm{~L} \\
\text { water }\end{array}$ & $\begin{array}{l}28 \mathrm{~g}(2 \mathrm{~T}) \text { to } 1 \mathrm{~L} \\
\text { water }\end{array}$ \\
\hline
\end{tabular}

\section{FOOD AND NUTRITION}

Malnutrition is \#1 risk to child health worldwide. It contributes to an estimated 2.5 M out of 6.3 M U5 deaths/year.

\section{A. Prediction and Early Warning Systems}

1. FEWS NET products

a. Food Security Outlook (FSO) - assumptions and 8-mo projections issued $3 \mathrm{x} /$ year

b. Food Security Outlook Update (FSOU) - update mid-term between FSOs

c. Key Messages (KM) - precedes FSO

d. Price Bulletin (PB)

e. Food Assistance Outlook Brief (FAOB) - regional outlook

2. FEWS NET distribution calendar

a. January - Key Messages (KM)

b. February - Food Security Outlook (FSO)

c. March - Key Messages (KM)

d. April - Food Security Outlook Update (FSOU)

e. May - Key Messages (KM)

f. June - Food Security Outlook (FSO)

g. July - Key Messages (KM)

h. August - Food Security Outlook Update (FSOU)

i. September - Key Messages (KM)

j. October - Food Security Outlook (FSO)

k. November - Key Messages (KM)

1. December - Food Security Outlook Update (FSOU) (optional)

\section{B. Protein-calorie Malnutrition}

1. Screening U5-mid-upper arm circumference (MUAC) used as a screening tool in pedes and adults. In pedes, $<13.5 \mathrm{~cm}$ or edema is referred for $\mathrm{W} / \mathrm{H}$ measurements. 
2. Individual diagnosis

a. pedes (U10)anthropometrics: $\Delta \mathrm{W} / \mathrm{H}$ before $\Delta \mathrm{W} /$ Age before $\Delta H /$ Age

Acute $\quad<->\quad$ Chronic

Table 7.3.8

Anthropometric Indicators in Children

\begin{tabular}{|c|c|c|c|}
\hline \multicolumn{2}{|c|}{ Combined anthropometrics } & \multicolumn{2}{|c|}{ W/H \% of median } \\
\hline & & $>80 \%(\mathrm{NL})$ & $<80 \%$ \\
\hline \multirow{2}{*}{$\begin{array}{l}\text { H/Age } \% \text { of } \\
\text { median }\end{array}$} & $>90 \%(\mathrm{NL})$ & $\mathrm{NL}$ & Wasted \\
\hline & $<90 \%$ & Stunted & Wasted \& stunted \\
\hline $\begin{array}{c}\text { Acute malnutrition } \\
\text { (Wasting) }\end{array}$ & $\begin{array}{c}\text { W/H Z score } \\
(\mathrm{WHZ})\end{array}$ & $\begin{array}{c}\text { W/H \% of median } \\
\text { (WHM) }\end{array}$ & $\begin{array}{c}\text { MUAC }(\mathrm{cm}) \\
(6-59 \mathrm{mo})\end{array}$ \\
\hline Mild acute & $-2 \leq z<-1$ & $\begin{array}{l}80 \% \leq \% \\
\mathrm{WHM}<90 \%\end{array}$ & $\begin{array}{l}12.5 \leq \text { MUAC } \\
<13.5\end{array}$ \\
\hline $\begin{array}{l}\text { Moderate acute } \\
(\mathrm{MAM})\end{array}$ & $-3 \leq z<-2$ & $\begin{array}{l}70 \% \leq \% \\
\mathrm{WHM}<80 \%\end{array}$ & $\begin{array}{l}11.5 \leq \mathrm{MUAC} \\
<12.5\end{array}$ \\
\hline $\begin{array}{l}\text { Severe acute } \\
\text { (SAM) }\end{array}$ & $\begin{array}{l}\mathrm{z}<-3 \text { or } \\
\text { symmetrical edema }\end{array}$ & $\begin{array}{l}\text { WHM }<70 \% \text { or } \\
\text { symmetrical edema }\end{array}$ & $\begin{array}{l}\text { MUAC }<11.5 \text { or } \\
\text { symmetrical edema }\end{array}$ \\
\hline $\begin{array}{l}\text { Global acute } \\
\text { (GAM) }\end{array}$ & $\begin{array}{l}\mathrm{z}<-2 \text { or } \\
\text { symmetrical edema }\end{array}$ & $\begin{array}{l}\text { WHM }<80 \% \text { or } \\
\text { symmetrical edema }\end{array}$ & $\begin{array}{l}\text { MUAC }<12.5 \text { or } \\
\text { symmetrical edema }\end{array}$ \\
\hline Underweight & $\begin{array}{c}\text { W/Age } \mathbf{Z} \text { score } \\
\text { (WAZ) }\end{array}$ & $\begin{array}{l}\text { W/Age \% of } \\
\text { median } \\
(\text { WAM })\end{array}$ & \\
\hline Mild & $-2 \leq \mathrm{z}<-1$ & & \\
\hline Moderate & $-3 \leq \mathrm{z}<-2$ & & \\
\hline Severe & $\begin{array}{l}\mathrm{z}<-3 \text { or } \\
\text { symmetrical edema }\end{array}$ & & \\
\hline $\begin{array}{c}\text { Chronic } \\
\text { malnutrition } \\
\text { (Stunting) }\end{array}$ & $\begin{array}{c}\text { H/Age } \mathbf{Z} \text { score } \\
\text { (HAZ) }\end{array}$ & $\begin{array}{c}\text { H/Age \% of } \\
\text { median } \\
(\mathrm{HAM})\end{array}$ & \\
\hline Mild stunting & $-2 \leq \mathrm{z}<-1$ & & \\
\hline $\begin{array}{l}\text { Moderately } \\
\text { stunted }\end{array}$ & $-3 \leq \mathrm{z}<-2$ & $\begin{array}{l}85 \% \leq \text { HAM } \\
<90 \%\end{array}$ & \\
\hline Severely stunted & $\begin{array}{l}\mathrm{z}<-3 \text { or } \\
\text { symmetrical } \\
\text { edema }\end{array}$ & $\begin{array}{l}\text { HAM }<85 \% \\
\text { or symmetrical } \\
\text { edema }\end{array}$ & \\
\hline
\end{tabular}

Note: in U2, length is the preferred term over height 


\section{Wasting}

- WHO defines 3 types of severe malnutrition: severe wasting (SAM), severe stunting, and edematous malnutrition. This last includes kwashiorkor and marasmatic kwashiorkor in the Wellcome classification.

- $\mathrm{SAM}=$ severe wasting cases or bilateral pitting edema cases (where due to malnutrition)

- $\mathrm{SAM}=\mathrm{WHZ}<-3, \mathrm{MUAC}<11.5 \mathrm{~cm}$, or bilateral pitting edema (WHO). WHM not in definition.

- SAM prevalence worldwide $\approx 20,000,000$.

- $\mathrm{SAM}$ mortality $\approx 9 \mathrm{x}$ mortality of normally nourished child and its CFR can be $10-50 \%$.

- $\mathrm{GAM}=\mathrm{MAM}+\mathrm{SAM}$

- $\mathrm{GAM}=$ moderate wasting cases, severe wasting cases, or bilateral pitting edema cases (where due to malnutrition)

\section{Underweight}

- Underweight is not used for screening or surveys in nutritional emergencies. It reflects past (chronic) and present (acute) undernutrition and is unable to distinguish between them. It encompasses children who are wasted and/or stunted. However, weight gain over time can be a sensitive indicator of growth faltering which is easily tracked on Road to Health charts.

\section{Stunting}

- Stunting generally occurs before age 2. It is irreversible.

- Stunting prevalence worldwide $\approx 165,000,000$.

- Stunting is not a good predictor of mortality, but the CFR from IDs in cases of severe stunting $\approx 3 \mathrm{x}$ the CFR from IDs in cases without stunting.

Reference standards can be absolute MUAC, centile, \% of median reference, or z scores:

- MUAC

Easy to understand. An excellent predictor of mortality. Permits comparisons between age groups insofar as the low growth velocity of MUAC in the U5 age group makes data roughly comparable. May be used alone in "quick-and-dirty" convenience samples to estimate local prevalence of wasting. However, not used alone in authoritative anthropometric surveys, and is commonly part of a two stage screening process to determine eligibility for feeding programs.

- Centiles

Easy to understand. Permits comparisons between age groups and outliers. However, data are not convenient to convert.

$\mathrm{Eg} \mathrm{z}-4.0=0.0032$ nd percentile

- \% of Median of reference population

WHM is the preferred indicator to determine eligibility for feeding programs (Sphere). Calculations are easy and are used in the WHO Road to Health Charts.

However, median reference data are not comparable between ages.

eg $60 \%$ wt-for-age $=$ severe malnutrition in infants

$=$ moderate malnutrition in school age kids 
Moreover, median reference data are not comparable between indicators.

eg $60 \%$ wt-for-age = severe malnutrition in infants

$60 \%$ wt-for-ht = death

- Z scores

Preferred indicator (Sphere, WHO) for reporting anthropometry survey results because it permits comparisons between age groups and nutritional indices.

However, data may be difficult to understand.

eg $\mathrm{z}$ score wt-for-age for $1 \mathrm{y} / \mathrm{o}$ :

$$
\begin{aligned}
& =\quad \underline{\mathrm{wt}}_{\mathrm{Pt}}-\mathrm{wt}_{\text {ref pop }} \\
& \mathrm{SD}_{\text {ref pop }} \\
& =\frac{6.1 \mathrm{~kg}-10.1 \mathrm{~kg}}{1.0} \\
& =\quad-4 \mathrm{SD} \text { below median for his age }
\end{aligned}
$$

\section{Overall}

WHZ gives higher prevalence of malnutrition than WHM for the same population. This is most marked where there is low baseline prevalence of disease, and especially for adolescents (who get subsequently over-referred).

WHZ is more statistically valid, but WHM is better predictor of mortality and is used for admission to TFCs.

Weight-for-age is influenced by weight-for-height and height-for-age. It can be difficult to interpret.

\begin{tabular}{|c|c|c|c|}
\hline $\begin{array}{c}\text { Nutritional } \\
\text { status }\end{array}$ & $\begin{array}{c}\text { BMI } \\
\text { (adults \& } \\
\text { adolescents) }\end{array}$ & $\begin{array}{c}\text { MUAC (cm) } \\
\text { (adults) }\end{array}$ & $\begin{array}{c}\text { MUAC (cm) } \\
\text { (pregnant \& } \\
\text { lactating) }\end{array}$ \\
\hline Obese & $30+$ & & \\
\hline Overweight & $25 \leq \mathrm{BMI}<30$ & & \\
\hline NL & $18.5 \leq \mathrm{BMI}<25$ & & \\
\hline Mild malnutrition & $17 \leq \mathrm{BMI}<18.5$ & & \\
\hline $\begin{array}{l}\text { Moderate } \\
\text { malnutrition }\end{array}$ & $16 \leq \mathrm{BMI}<17$ & $\begin{array}{l}16.0 \leq \text { MUAC } \\
<18.5\end{array}$ & $\begin{array}{l}17.0 \leq \text { MUAC } \\
<18.5\end{array}$ \\
\hline $\begin{array}{l}\text { Severe } \\
\text { malnutrition }\end{array}$ & $\begin{array}{l}\mathrm{BMI}<16 \text { or edema } \\
\text { or }<5 \% \text { of } 10-18 \mathrm{yr} \\
\text { reference population }\end{array}$ & $\begin{array}{l}\text { MUAC }<16.0 \text { or } \\
\text { edema }\end{array}$ & $\begin{array}{l}\text { MUAC }<17.0 \text { or } \\
\text { edema }\end{array}$ \\
\hline $\begin{array}{l}\text { Global } \\
(\bmod +\text { severe })\end{array}$ & $\mathrm{BMI}<17$ or edema & $\begin{array}{l}\text { MUAC }<18.5 \text { or } \\
\text { edema }\end{array}$ & $\begin{array}{l}\text { MUAC }<18.5 \text { or } \\
\text { edema }\end{array}$ \\
\hline
\end{tabular}

b. adults and adolescents (O10) anthropometrics: $\quad \mathrm{BMI}=$ weight $(\mathrm{kg}) /$ height $(\mathrm{m})^{2}$

Table 7.3.9

Anthropometric Indicators in Adults 
3. Population prevalence (U5)

a. survey key attributes

(1) representative at unit of analysis

(2) robust ( $>25$ clusters recommended)

(3) standardized (standardization test done)

(4) data normally distributed (check standard deviation)

(5) findings plausible (plausibility score)

b. preferences for indicator and methods (IPC)

(1) GAM by WHZ from representative survey $>$

(2) GAM by WHZ from sentinel sites >

(3) GAM by MUAC from representative survey $>$

(4) GAM by MUAC from exhaustive screening $>$

(5) GAM by MUAC from sentinel sites $>$

(6) GAM by MUAC from screening

\section{Malnutrition Classification}

Table 7.3.10

Malnutrition Classification by Prevalence Range (WHO [5])

\begin{tabular}{|l|l|l|l|l|}
\hline \multirow{2}{*}{ Malnutrition } & \multicolumn{4}{|c|}{ Prevalence (\%) } \\
\cline { 2 - 5 } & \multicolumn{1}{|c|}{$\begin{array}{c}\text { Acceptable } \\
\text { (Low) }\end{array}$} & \multicolumn{1}{c|}{$\begin{array}{c}\text { Poor } \\
\text { (Med) }\end{array}$} & $\begin{array}{c}\text { Serious } \\
\text { (High) }\end{array}$ & $\begin{array}{c}\text { Critical } \\
\text { (Very high) }\end{array}$ \\
\hline Wasting (GAM) & $<5$ & $5-9$ & $10-14$ & $\geq 15$ \\
\hline Underweight & $<10$ & $10-19$ & $20-29$ & $\geq 30$ \\
\hline Stunting & $<20$ & $20-29$ & $30-39$ & $\geq 40$ \\
\hline
\end{tabular}

Source: World Health Organization. In: Global Database on Child Growth and Malnutrition (c) 2017. Used with permission of WHO.

Table 7.3.11

Malnutrition Classification by Prevalence Range (adapted from IPC [6])

\begin{tabular}{|l|l|l|l|l|l|}
\hline \multirow{2}{*}{ Indicator } & \multicolumn{5}{|c|}{ Prevalence (\%) } \\
\cline { 2 - 6 } & $\begin{array}{c}\text { Phase 1 } \\
\text { Acceptable }\end{array}$ & $\begin{array}{c}\text { Phase 2 } \\
\text { Alert }\end{array}$ & $\begin{array}{c}\text { Phase 3 } \\
\text { Serious }\end{array}$ & $\begin{array}{c}\text { Phase 4 } \\
\text { Critical }\end{array}$ & $\begin{array}{c}\text { Phase 5 } \\
\text { Ext critical }\end{array}$ \\
\hline $\begin{array}{l}\text { GAM by WHZ } \\
\%<-2 \text { or edema }\end{array}$ & $<5$ & $5-9.9$ & $10-14.9$ & $15-29.9$ & $\geq 30$ \\
\hline $\begin{array}{l}\text { GAM by MUAC } \\
\%<12.5 \text { cm or } \\
\text { edema }\end{array}$ & $<6$ & $6-10.9$ & $11-16.9$ & $\geq 17$ \\
\hline
\end{tabular}

Source: IPC Global Partners (C) 2016. Used with permission of IPC Global Partnership 
Acute Food Security Classification

Table 7.3.12

Acute Food Security Reference Table (IPC [7])

\begin{tabular}{|c|c|}
\hline Phase classification & $\begin{array}{c}\text { Reference data } \\
\text { (not all indicators required to determine } \\
\text { classification) }\end{array}$ \\
\hline $\begin{array}{l}\text { Phase 1-Minimal } \\
\text { Generally food secure } \\
\text { (green) }\end{array}$ & $\begin{array}{l}\mathrm{CDR}<0.5 / 10,000 / \mathrm{d}, \mathrm{U} 5 \mathrm{DR} \leq 1 / 10,000 / \mathrm{d} \\
\mathrm{GAM}<5 \% \\
\text { Food consumption usually adequate }(> \\
2100 \mathrm{kcal} / \mathrm{p} / \mathrm{d}) \\
\text { Livelihood assets sustainable utilization }\end{array}$ \\
\hline $\begin{array}{l}\text { Phase 2-Stressed } \\
\text { Borderline food insecure } \\
\text { (yellow) }\end{array}$ & $\begin{array}{l}\text { CDR }<0.5 / 10,000 / \mathrm{d}, \mathrm{U} 5 \mathrm{DR} \leq 1 / 10,000 / \mathrm{d} \\
\text { GAM } 5-10 \% \\
\text { Food consumption borderline adequate } \\
(2100 \mathrm{kcal} / \mathrm{p} / \mathrm{d}) \\
20 \%+\text { of } \mathrm{HH} \text { c livelihood assets stressed \& } \\
\text { unsustainable utilization (livelihood deficit) } \\
\text { Non-food expenditures (schools, health care) } \\
\text { decrease }\end{array}$ \\
\hline $\begin{array}{l}\text { Phase 3-Crisis } \\
\text { Acute food and livelihood crisis } \\
\text { (orange) }\end{array}$ & $\begin{array}{l}\text { CDR } 0.5-1 / 10,000 / \mathrm{d}, \text { U5DR } 1-2 / 10,000 / \mathrm{d} \\
\text { GAM } 10-15 \% \text { or greater than usual and } \\
\text { increasing } \\
20 \%+\text { of } \mathrm{HH} \text { c food consumption gaps } \\
(<2100 \mathrm{kcal} / \mathrm{p} / \mathrm{d} \text { or } 2100 \mathrm{kcal} / \mathrm{p} / \mathrm{d} \text { via asset } \\
\text { stripping) (survival deficit) } \\
\text { Livelihood assets accelerated \& critical } \\
\text { depletion }\end{array}$ \\
\hline $\begin{array}{l}\text { Phase 4-Emergency } \\
\text { (red) }\end{array}$ & $\begin{array}{l}\text { CDR } 1-2 / 10,000 / \mathrm{d}, \mathrm{U} 5 \mathrm{DR}>2-4 / 10,000 / \mathrm{d} \\
\text { (excess mortality) } \\
\text { GAM }>15-30 \% \text { or greater than usual and } \\
\text { increasing } \\
20 \%+\text { of } \mathrm{HH} \text { c food consumption gaps marked } \\
(<2100 \mathrm{kcal} / \mathrm{p} / \mathrm{d}) \\
\text { Livelihood assets near complete \& irreversible } \\
\text { depletion }\end{array}$ \\
\hline Phase 5-Famine (maroon) & $\begin{array}{l}\text { CDR }>2 / 10,000 / \mathrm{d}, \mathrm{U} 5 \mathrm{DR}>4 / 10,000 / \mathrm{d} \\
\mathrm{GAM}>30 \% \\
20 \%+\text { of } \mathrm{HH} \text { c food consumption grossly } \\
\text { inadequate }(<<2100 \mathrm{kcal} / \mathrm{p} / \mathrm{d}) \\
\text { Livelihood assets complete loss }\end{array}$ \\
\hline
\end{tabular}

Source: IPC Global Partners (C) 2012. Used with permission of FAO-UN on behalf of IPC Global Partnership

\section{Therapy}

IPC Phase 2 needs livelihood support.

IPC Phase $3+$ needs above plus nutritional support. 
Table 7.3.13

Nutritional Support of Malnourished Population

\begin{tabular}{|l|l|}
\hline \multicolumn{1}{|c|}{ Nutritional status } & \multicolumn{1}{c|}{ Therapy } \\
\hline Mild acute & Adequate general ration \\
\hline $\begin{array}{l}\text { Moderate acute } \\
(\text { GAM }>10 \%)\end{array}$ & $\begin{array}{l}\text { Above + supplementary feeding or } \\
\text { community therapeutic care (U5 + PLW) }\end{array}$ \\
\hline $\begin{array}{l}\text { Severe acute } \\
(\text { SAM }>2 \%)\end{array}$ & $\begin{array}{l}\text { Therapeutic feeding, community therapeutic } \\
\text { care, or hospitalization }\end{array}$ \\
\hline
\end{tabular}

\section{General Food Distribution (GFD)}

$2100 \mathrm{kcal} / \mathrm{p} / \mathrm{d}$ requires $12 \mathrm{~kg}$ food $/ \mathrm{p} / \mathrm{mo}$ or $\approx 15 \mathrm{~kg}$ food $/ \mathrm{p} / \mathrm{mo}$ in logistics pipeline.

Ration - WFP commonly aims for $50 \%$ ration in settings where resources are limited.

Cost $-\$ 1000 /$ MT Title II bulk food from US sources

Preventive Intervention (for populations on edge of frank malnutrition)

Targeted-see SFP below

\section{Community Management of Acute Malnutrition (CMAM)}

4 Components:

\section{Stabilization Center (SC)}

admission criteria: age 6-59 mo, SAM (MUAC $<11.5 \mathrm{~cm}, \mathrm{WHZ}<-3$, or edema), anorexia, or severe medical complications

treatment: F75, F100, IVF, ABX

discharge criteria: appetite recovered, medical complication improved, pitting edema decreased, and clinically well

Outpatient Therapeutic Program (OTP)

admission criteria: age $6-59 \mathrm{mo}$, SAM (MUAC $<11.5 \mathrm{~cm}$ ), appetite intact, and no severe medical complications

treatment: RUTF (eg Plumpy'Nut ${ }^{\circledR}$ nutritionally = F100)

discharge criteria: $15 \%$ weight gain (from entry weight), no bilateral pitting edema $\times 2$ wks, and 2 mo minimum observation/treatment period

Supplementary Feeding Program (SFP)

blanket-all $\mathrm{HH}$ in geographically targeted catchment area (eg where IPC $3+$ and GAM $>15 \%$ or $10-14 \%$ with aggravating factors)

targeted-some $\mathrm{HH}$ in catchment area (eg where GAM 10-14\% or 5-9\% with aggravating factors); U5 and pregnant or lactating women vs U5 alone vs U2 alone depending on resources available and challenges with case finding) 
overall programmatic target $-50 \%$ coverage for SAM in rural areas

(Sphere); $30 \%$ coverage for MAM in rural areas

admission criteria

pedes: age $6-59 \mathrm{mo}, \mathrm{MUAC}<12.5 \mathrm{~cm}$, with appetite, discharged from

OTP, no severe medical complications

pregnant \& lactating: MUAC $<21.0 \mathrm{~cm}$, and 2nd - 3rd trimester or with infant $<6$ mo

treatment: RUSF as dry rations eg Plumpy'Sup ${ }^{\circledast}, \mathrm{CSB}, \mathrm{CSB}+($ supercereal), CSB ++ (supercereal +)

NB CSB may also be cooked on-site in emergency school feedings. discharge criteria (pedes): weight gain, $M U A C>12.5 \mathrm{~cm}$, time in program $>2$ months

\section{Community Outreach with Mobile Brigades}

\section{Therapeutic Feeding Program}

Admission criteria for U5: SAM ( $\mathrm{WHZ}<-3$, MUAC $<11.5 \mathrm{~cm}$, or bilateral pitting edema)

Discharge criteria for U5: WHZ $>-2.5$, no edema, and clinically well (generally takes 4-6 weeks)

Treatment protocol (WHO, ICDDR)

Shock Severe dehydration: RL + D5, 1/2 strength Darrow's + D5, or $1 / 2 \mathrm{NS}+\mathrm{D} 5$

Dose: $100 \mathrm{cc} / \mathrm{kg} \mathrm{IV}$

Table 7.3.14

IV Therapy of Severe Dehydration

\begin{tabular}{|l|l|l|}
\hline \multicolumn{1}{|c|}{ Age } & \multicolumn{1}{c|}{ Initial bolus } & \multicolumn{1}{c|}{ Balance } \\
\hline$<1$ year & $30 \mathrm{cc} / \mathrm{kg}$ IV over $1 \mathrm{~h}$ & $70 \mathrm{cc} / \mathrm{kg}$ IV over $5 \mathrm{~h}$ \\
\hline$>1$ year & $30 \mathrm{cc} / \mathrm{kg}$ IV over $1 / 2 \mathrm{~h}$ & $70 \mathrm{cc} / \mathrm{kg}$ IV over $2 \frac{1 / 2}{\mathrm{~h}}$ \\
\hline $\begin{array}{l}\text { If severely malnourished } \\
(\text { WHO) }\end{array}$ & $\begin{array}{l}15 \mathrm{cc} / \mathrm{kg} \text { IV over } \\
1 \mathrm{~h}+\text { ReSoMal } 10 \mathrm{cc} / \mathrm{kg} \\
\text { by NGT }\end{array}$ & $\begin{array}{l}\text { If improved after initial } \\
\text { bolus, repeat } \\
15 \mathrm{cc} / \mathrm{kg} \text { IV over } \\
1 \mathrm{~h}+\text { ReSoMal } 10 \mathrm{cc} / \mathrm{kg} / \mathrm{h} \\
\text { PO/NGT } \mathrm{x} 10 \mathrm{~h} ; \\
\text { If not improved after } \\
\text { initial bolus, assume septic } \\
\text { shock }\end{array}$ \\
\hline $\begin{array}{l}\text { If severely } \\
\text { malnourished (ICDDR) }\end{array}$ & & $\begin{array}{l}10 \mathrm{cc} / \mathrm{kg} / \mathrm{h} \text { over } 10 \mathrm{~h}+\mathrm{ORS} \\
5-10 \mathrm{cc} / \mathrm{kg} \text { PO after each } \\
\text { loose stool }\end{array}$ \\
\hline
\end{tabular}


Dehydration

Moderate dehydration (5-10\%): ReSoMal PO or NGT

With malnutrition: Dose $75-100 \mathrm{cc} / \mathrm{kg}$ PO/NGT over $12 \mathrm{~h}$ given as $5 \mathrm{cc} / \mathrm{kg} / \mathrm{half} \mathrm{h} \times 4$, then $5-10 \mathrm{cc} / \mathrm{kg} / \mathrm{h} \times 10 \mathrm{~h}(\mathrm{WHO})$

Without malnutrition: Dose $75-100 \mathrm{cc} / \mathrm{kg}$ PO over $4 \mathrm{~h}$ (if age $>1 \mathrm{yr}$ ) or over $6 \mathrm{~h}$ (if $<$ age $1 \mathrm{yr})$ (ICDDR)

NB ICDDR does not use ReSoMal but prefers rice-based ORS for all patients with diarrhea

Feeding c therapeutic milks -F75 (Phase 1), F100 (Phase 2)

D10 or F75 for hypoglycemia; blankets/warmer for hypothermia $3 \mathrm{Rx}-\mathrm{ABX}$, mebendazole, antimalarial

Adjuncts - vitamins A-D, minerals, measles vaccine, Fe (only in rehab phase)

a. process indicators

(1) daily average weight gain at TFC: $>8 \mathrm{~g} / \mathrm{kg} / \mathrm{p}$

b. outcome indicators

(1) cure

(2) complications ( $25 \%$ SAM will have medical complications)

(3) death ( 25\% SAM will die with good care, and 50\% will die with mediocre care)

Table 7.3.15

Exit Indicators in Therapeutic Feeding Programs

\begin{tabular}{|c|c|c|c|c|c|}
\hline \multicolumn{2}{|c|}{ Setting/reference } & \multicolumn{4}{|c|}{ Exit indicator } \\
\hline & & \multirow{2}{*}{$\begin{array}{r}\text { Cure } \\
>75 \%\end{array}$} & \multirow{2}{*}{$\begin{array}{l}\text { Mortality } \\
<10 \%\end{array}$} & \multirow{2}{*}{$\begin{array}{l}\text { Default } \\
<15 \%\end{array}$} & \multirow[t]{2}{*}{ Non-response } \\
\hline TFC & $\begin{array}{l}\text { Target } \\
\text { (SPHERE) }\end{array}$ & & & & \\
\hline & $\begin{array}{l}\text { Target } \\
(\mathrm{NGO})\end{array}$ & $>80 \%$ & $<5 \%$ & $<10 \%$ & \\
\hline & $\begin{array}{l}\text { Alert } \\
(\mathrm{NGO})\end{array}$ & $<50 \%$ & $>15 \%$ & $>25 \%$ & \\
\hline \multirow[t]{3}{*}{ SFC } & $\begin{array}{l}\text { Target } \\
\text { (SPHERE) }\end{array}$ & $>75 \%$ & $<3 \%$ & $<15 \%$ & \\
\hline & $\begin{array}{l}\text { Target } \\
\text { (NGO) }\end{array}$ & $>75 \%$ & $<3 \%$ & $<15 \%$ & $<20 \%$ \\
\hline & \begin{tabular}{|l} 
Alert \\
$(\mathrm{NGO})$
\end{tabular} & $<50 \%$ & $>10 \%$ & $>30 \%$ & $>30 \%$ \\
\hline
\end{tabular}


C. Micronutrient Deficiency

Table 7.3.16

Micronutrient deficiency states

\begin{tabular}{|c|c|c|}
\hline Micronutrients & Disease & Clinical findings \\
\hline \multicolumn{3}{|l|}{ Vitamins } \\
\hline A & Avitamnosis A & $\begin{array}{l}\text { Night blindness, xerophthalmia, Bitot's } \\
\text { spots, corneal ulcer/ keratomalacia, } \\
\text { corneal scars }\end{array}$ \\
\hline $\mathrm{B}_{1}$ (Thiamine) & Beri-beri & $\begin{array}{l}\text { Wet (acute)-congestive heart failure, } \\
\text { anasarca; dry (chronic) - peripheral } \\
\text { neuropathy (eg sensory } \Delta \text { s, wrist drop), } \\
\text { weakness, weight loss, progressive } \\
\text { ascending weakness (can't stand from a } \\
\text { squat) }\end{array}$ \\
\hline $\mathrm{B}_{2}$ (Riboflavin) & & Cheilosis, angular stomatitis, glossitis \\
\hline $\mathrm{B}_{3}($ Niacin $)$ & Pellagra & $\begin{array}{l}\text { Dermatitis (sun-exposed areas eg Casal's } \\
\text { necklace; tongue bright red), diarrhea, } \\
\text { dementia, death }\end{array}$ \\
\hline $\mathrm{B}_{9}$ (Folate) & Anemia & Glossitis, others as per anemia below \\
\hline $\mathrm{C}$ & Scurvy & $\begin{array}{l}\text { Gingivitis, dental loosening, petechiae, } \\
\text { delayed wound healing }\end{array}$ \\
\hline $\mathrm{D}$ & Rickets & $\begin{array}{l}\text { Bone-cartilage junctions enlarged at ribs } \\
\text { (rachitic beads), wrists, \& ankles; frontal } \\
\text { bossing; sternal prominence; long bones } \\
\text { bowed; joints with "splaying, cupping, \& } \\
\text { fraying" }\end{array}$ \\
\hline \multicolumn{3}{|l|}{ Minerals } \\
\hline I & Hypothyroid & $\begin{array}{l}\text { Goiter, cretinism (mental retardation, } \\
\text { short stature, squint, deaf-mutism) }\end{array}$ \\
\hline $\mathrm{Zn}$ & Zinc deficiency & Diarrhea, delayed wound healing \\
\hline \multicolumn{3}{|l|}{ Other Cofactors } \\
\hline $\mathrm{Fe}$ & Anemia & $\begin{array}{l}\text { Fatigue, dyspnea, pallor, mental dullness } \\
\text { (\#1 nutritional disorder in the world) }\end{array}$ \\
\hline
\end{tabular}


VIII. CHEMICAL WEAPONS

A. Chemical Agents

Table 7.3.17

Chemical Agent Characteristics, Clinical Effects, and Treatments

\begin{tabular}{|c|c|c|c|c|c|}
\hline \multicolumn{6}{|c|}{ Chemical agents } \\
\hline Agent & $\begin{array}{c}\text { Physical } \\
\text { characteristics }\end{array}$ & $\begin{array}{l}\text { Systems } \\
\text { affected }\end{array}$ & $\mathbf{S x}$ & $\begin{array}{c}\text { Decon first } \\
\text { aid }\end{array}$ & Tx antidote \\
\hline $\begin{array}{l}\text { Blister } \\
\text { Mustard } \\
(\mathrm{H}, \mathrm{HD}, \\
\mathrm{HN})\end{array}$ & $\begin{array}{l}\text { Oily light } \\
\text { yellow-brown } \\
\text { liquids with } \\
\text { odor of garlic }\end{array}$ & \begin{tabular}{|l|} 
Eye-few \\
hrs; resp \& \\
derm-2- \\
24 hrs; lethal \\
in large doses
\end{tabular} & $\begin{array}{l}\text { Red skin, } \\
\text { blisters, eye } \\
\text { burning, } \\
\text { coughing }\end{array}$ & $\begin{array}{l}\text { Remove } \\
\text { agent, } \\
\text { S\&W, } \\
\text { flush with } \\
\text { dilute } \\
\text { bleach }\end{array}$ & \begin{tabular}{|l|} 
None, \\
supportive \\
care
\end{tabular} \\
\hline $\begin{array}{l}\text { Blister } \\
\text { Lewisite } \\
\text { (L) }\end{array}$ & $\begin{array}{l}\text { Oily colorless } \\
\text { liquid with odor } \\
\text { of geraniums }\end{array}$ & $\begin{array}{l}\text { Derm- } \\
\text { immediate } \\
\text { pain; lethal in } \\
\text { large doses }\end{array}$ & $\begin{array}{l}\text { Skin pain } \\
\text { or irritation, } \\
\text { eye burning, } \\
\text { coughing }\end{array}$ & $\begin{array}{l}\text { Remove } \\
\text { agent, } \\
\text { S\&W, } \\
\text { flush with } \\
\text { dilute } \\
\text { bleach }\end{array}$ & $\begin{array}{l}\text { BAL, } \\
\text { supportive } \\
\text { care }\end{array}$ \\
\hline $\begin{array}{l}\text { Blister } \\
\text { Phosgene } \\
\text { oxime } \\
(\mathrm{CX})\end{array}$ & $\begin{array}{l}\text { Solid }<95^{\circ} \mathrm{F} \\
\text { but vaporizes }\end{array}$ & $\begin{array}{l}\text { Derm- } \\
\text { immediate } \\
\text { pain lethal in } \\
\text { large doses }\end{array}$ & $\begin{array}{l}\text { Skin pain, } \\
\text { wheals, eye } \\
\text { burning, } \\
\text { coughing }\end{array}$ & $\begin{array}{l}\text { Remove } \\
\text { agent, } \\
\text { S\&W, } \\
\text { flush with } \\
\text { dilute } \\
\text { bleach }\end{array}$ & $\begin{array}{l}\text { None, } \\
\text { supportive } \\
\text { care }\end{array}$ \\
\hline $\begin{array}{l}\text { Blood } \\
\text { Cyanide } \\
(\mathrm{AC})\end{array}$ & $\begin{array}{l}\text { Rapid } \\
\text { evaporating } \\
\text { liquids with } \\
\text { odor of bitter } \\
\text { almonds; gas } \\
\text { lighter than air }\end{array}$ & $\begin{array}{l}\text { Resp, } \\
\text { heme-death } \\
\text { in min; highly } \\
\text { lethal }\end{array}$ & $\begin{array}{l}\text { Cherry } \\
\text { red skin or } \\
\text { lips, rapid } \\
\text { respirations, } \\
\text { dizziness, HA, } \\
\text { seizures, death }\end{array}$ & $\begin{array}{l}\text { Usually } \\
\text { none; } \\
\text { leave area, } \\
\text { aeration, } \\
\text { S\&W }\end{array}$ & $\begin{array}{l}\text { Amyl } \\
\text { nitrite, } \mathrm{Na} \\
\text { thiosulfate }\end{array}$ \\
\hline $\begin{array}{l}\text { Blood } \\
\text { Cyanogen } \\
\mathrm{Cl}(\mathrm{CK})\end{array}$ & $\begin{array}{l}\text { Rapid } \\
\text { evaporating } \\
\text { liquids with } \\
\text { odor of bitter } \\
\text { almonds; gas } \\
\text { heavier than air }\end{array}$ & $\begin{array}{l}\text { Resp, } \\
\text { heme-death } \\
\text { in min; highly } \\
\text { lethal }\end{array}$ & $\begin{array}{l}\text { Cherry } \\
\text { red skin or } \\
\text { lips, rapid } \\
\text { respirations, } \\
\text { dizziness, HA, } \\
\text { seizures, death }\end{array}$ & $\begin{array}{l}\text { Usually } \\
\text { none; } \\
\text { leave area, } \\
\text { aeration, } \\
\text { S\&W }\end{array}$ & $\begin{array}{l}\text { Amyl } \\
\text { nitrite, } \mathrm{Na} \\
\text { thiosulfate }\end{array}$ \\
\hline $\begin{array}{l}\text { Choking } \\
\text { Phosgene } \\
(\mathrm{CG})\end{array}$ & $\begin{array}{l}\text { Rapid } \\
\text { evaporating } \\
\text { liquid with } \\
\text { odor of mown } \\
\text { hay }\end{array}$ & $\begin{array}{l}\text { Resp, derm-- } \\
\text { death in days; } \\
\text { lethal in } \\
\text { large doses }\end{array}$ & $\begin{array}{l}\text { Eye and resp } \\
\text { irritation }\end{array}$ & $\begin{array}{l}\text { Leave } \\
\text { area, } \\
\text { aeration, } \\
\text { S\&W, eye } \\
\text { irrigation }\end{array}$ & $\begin{array}{l}\text { None, } \\
\text { supportive } \\
\text { care }\end{array}$ \\
\hline
\end{tabular}




\begin{tabular}{|c|c|c|c|c|c|}
\hline \multicolumn{6}{|c|}{ Chemical agents } \\
\hline Agent & $\begin{array}{c}\text { Physical } \\
\text { characteristics }\end{array}$ & $\begin{array}{l}\text { Systems } \\
\text { affected }\end{array}$ & $\mathbf{S x}$ & $\begin{array}{c}\text { Decon first } \\
\text { aid }\end{array}$ & Tx antidote \\
\hline $\begin{array}{l}\text { Choking } \\
\text { Chlorine } \\
(\mathrm{Cl})\end{array}$ & $\begin{array}{l}\text { Gas at room } \\
\text { temp }\end{array}$ & $\begin{array}{l}\text { Resp-death } \\
\text { in days; lethal } \\
\text { in large doses }\end{array}$ & $\begin{array}{l}\text { Eye \& resp } \\
\text { irritation, } \\
\text { chest tightness, } \\
\text { delayed pulm } \\
\text { edema }\end{array}$ & $\begin{array}{l}\text { Leave } \\
\text { area, } \\
\text { aeration, } \\
\text { S\&W, eye } \\
\text { irrigation }\end{array}$ & $\begin{array}{l}\text { None, } \\
\text { supportive } \\
\text { care }\end{array}$ \\
\hline $\begin{array}{l}\text { Nerve } \\
\text { Tabun } \\
\text { (GA), } \\
\text { Sarin } \\
\text { (GB), } \\
\text { Soman } \\
\text { (GD) }\end{array}$ & $\begin{array}{l}\text { Colorless, } \\
\text { odorless liquid; } \\
\text { G agents less } \\
\text { volatile than } \\
\text { water }\end{array}$ & $\begin{array}{l}\text { Resp- } \\
\text { seconds to } \\
\text { minutes; } \\
\text { derm - min } \\
\text { to hrs; highly } \\
\text { lethal }\end{array}$ & $\begin{array}{l}\text { Blurry vision, } \\
\text { twitching, chest } \\
\text { tightness, SOB } \\
\text { SLUDGE, } \\
\text { brady,broncho- } \\
\text { spasm, seizures }\end{array}$ & $\begin{array}{l}\text { Remove } \\
\text { agent, } \\
\text { S\&W, } \\
\text { flush with } \\
\text { dilute } \\
\text { bleach }\end{array}$ & $\begin{array}{l}\text { Atropine, } \\
\text { 2-PAM }\end{array}$ \\
\hline $\begin{array}{l}\text { Nerve } \\
\text { VX }\end{array}$ & $\begin{array}{l}\text { Slight yellow } \\
\text { colored liquid } \\
\text { at room temp; } \\
\text { V agents as } \\
\text { volatile as } \\
\text { motor oil }\end{array}$ & $\begin{array}{l}\text { Resp- } \\
\text { seconds to } \\
\text { minutes; } \\
\text { derm-min } \\
\text { to hrs; highly } \\
\text { lethal }\end{array}$ & $\begin{array}{l}\text { Blurry vision, } \\
\text { twitching, chest } \\
\text { tightness, SOB } \\
\text { SLUDGE, } \\
\text { brady,broncho- } \\
\text { spasm, seizures }\end{array}$ & $\begin{array}{l}\text { Remove } \\
\text { agent, } \\
\text { S\&W, } \\
\text { flush with } \\
\text { dilute } \\
\text { bleach }\end{array}$ & $\begin{array}{l}\text { Atropine, } \\
\text { 2-PAM }\end{array}$ \\
\hline $\begin{array}{l}\text { Riot } \\
\text { control } \\
\text { Tear gas } \\
\text { (CS), } \\
\text { Mace } \\
\text { (CN) }\end{array}$ & $\begin{array}{l}\text { Gas at room } \\
\text { temp }\end{array}$ & $\begin{array}{l}\text { Resp- } \\
\text { seconds; } \\
\text { non-lethal }\end{array}$ & $\begin{array}{l}\text { Eye \& resp } \\
\text { irritation }\end{array}$ & $\begin{array}{l}\text { Leave } \\
\text { area, } \\
\text { aeration, } \\
\text { S\&W, eye } \\
\text { irrigation }\end{array}$ & None \\
\hline
\end{tabular}

BAL British anti-Lewisite

2-PAM 2-pyridine aldoxime methyl chloride

S\&W soap and water

SLUDGE salivation, lacrimation, urination, defecation, GI distress, emesis SOB shortness of breath

B. Management of MCI from Suspected Chemical Weapons

(adapted from Domestic Preparedness Training [8])

Overarching rule: all responders should wear personal protective equipment to avoid becoming a victim.

\section{Notification Phase (RAIN)}

1. Recognize a problem
a. mass casualties
b. syndromic casualty pattern
c. dissemination device
d. warning from perpetrator
e. patient (host)-compelling symptoms
f. agent-identifiable sights or smells
g. environment-dead animals 
2. Avoid

a. distance $-100 \mathrm{~m}$ from chemical attack, $300 \mathrm{~m}$ from explosion

b. direction - upwind, upgrade, upstream

c. 4 don'ts - don't become a victim, don't rush in, don't TEST (taste, eat, smell, touch), don't assume anything

3. Isolate

a. cordon off the area to extent possible

b. isolate the casualties

4. Notify authorities

a. informant

b. agent released

c. immediate $\mathrm{M} \& \mathrm{M}$

d. signs and symptoms

e. type of device/vehicle/container-specify sights, smells, sounds

f. secondary disaster of fire or explosion

g. wind direction, weather conditions

h. witnesses

i. lead responder

j. actions taken

k. actions forthcoming

1. meeting place for responders

m. follow-up contact (time, means, channel)

\section{Response Phase}

1. Site security

a. position equipment upwind, upgrade, and upstream from incident site

b. isolate the area including downwind vapor hazard area

c. establish hot (ambient hazard), warm (contaminated victims), and cold (clean treatment) zones

2. Staff protection
a. PPE for providers in the hot zone
b. ICS to manage the incident
c. SOPs to guide responder actions

3. Social controls (PINS)
a. Preserve evidence
b. Identify the agent
c. Neutralize contaminated areas
d. Search for secondary devices

4. Environmental and case management

a. corral casualties and victims

b. establish decontamination stations

c. perform decontamination

d. provide first aid-triage, treat, transport 


\section{Emergency Self-decontamination}

1. Wet or blot (blotting for chemical contamination; wetting down for bio or nuclear contamination)

2. Strip off clothing

3. Flush the affected area with water or dilute bleach

4. Cover the affected area

\section{Implications for Disaster Management}

1. Forensic

2. Clinical

3. Epidemiological

4. Pharmacological

5. Environmental

6. Social

7. Judicial

8. Political

\section{EPI METHODS}

\section{A. Rates}

1. Death rates - calculated incidence of death expressed per $10,000 \mathrm{p} / \mathrm{d}$ or per $1000 \mathrm{p} / \mathrm{mo}$; data collected by retrospective surveys (eg 3 month period) to gauge severity of public health emergency particularly where sudden events lead to spike in mortality

a. CDR - crude death rate

b. ASDR - age-specific death rate (eg U5DR or death rate of children 0-5 yr) during a studied time interval (written as ${ }_{5} \mathrm{M}_{0}$ or 0-5DR); age of study cohort, eg 0-5 yr, should not be confused with study time intervals

Table 7.3.18

\section{Representative death rates}

\begin{tabular}{|l|l|l|}
\hline \multicolumn{1}{|c|}{ Situation } & \multicolumn{1}{c|}{ CDR } & \multicolumn{1}{c|}{-5DR } \\
\hline Baseline in developing countries & $<<1$ & 1 \\
\hline Emergency under control & $<1$ & $<2$ \\
\hline Serious trouble & $1-2$ & $2-4$ \\
\hline Emergency out of control & $>2$ & $>4$ \\
\hline Catastrophe & $>4$ & \\
\hline
\end{tabular}

2. Mortality rates - calculated probability of dying before a specified age expressed per 1000 live births; data collected by national health authorities in periodic (annual) demographic surveys to reflect ongoing health status

a. CMR - calculated probability of mortality in given population for specific time 
b. IMR - calculated probability of a live borne child dying before $1 \mathrm{yr}$

c. U5MR - calculated probability of a live borne child dying before $5 \mathrm{yr}$

NB MR $\neq$ DR. Eg CMR $\neq$ CDR, U5MR $\neq$ U5DR. Different rates measure different things and are not directly comparable.

However, MRs may be converted into DRs by the following:

CDR or U5DR $($ deaths $/ 10,000 / d)=-\ln (1-\mathrm{p} / 1000) \times 5.47$

where $\mathrm{p}=\mathrm{CMR}$ or U5MR (deaths/1000 live births). However, this has little field utility.

NB MMR - maternal mortality ratio has different units in numerators (maternal deaths) and denominators (live births), thus is a ratio, not a rate

\section{B. Conclusions}

Interpolation

The application of study findings to an entire population from which the sample was drawn. If the survey was well-conducted, the results may be considered representative of the entire population. This is scientifically justified. However a confidence interval should accompany any parameter estimate of that population.

Extrapolation

The extension of study findings to a population or period which was not represented in the sample. It works by association -if 2 populations appear to be experiencing similar conditions, the morbidity/mortality experience of one may be imputed to the other. This is not scientifically justified, but is often done where data are insufficient or impossible to collect.

\section{References}

1. Declaration of Alma-Ata. International Conference on Primary Health Care, AlmaAta, USSR, 6-12 September 1978. Available from: http://www.who.int/publications/ almaata_declaration_en.pdf?ua $=1$.

2. Walsh J, Warren K. Selective primary health care-an interim strategy for disease control in developing countries. N Engl J Med 1979; 301:967-74.

3. Mara D, Feachem RG. Water and excreta-related diseases: unitary environmental classification. Journal of Environmental Engineering, April 1999; 125(4):334-9.

4. Feachem RG. Infections related to water and excreta: the health dimension of the decade. In: Water Supply and Sanitation in Developing Countries. London: Institution of Water Engineers and Scientists, 1983.

5. World Health Organization. Global database on child growth and malnutrition. Available from: https://www.who.int/nutgrowthdb/about/introduction/en/index5. html. Accessed June 9, 2017. 
6. IPC Global Partners. Addendum to IPC Technical Manual Version 2.0. Tools and procedures for classification of acute malnutrition. Rome: IPC Global Partnership, 2016. p 14.

7. IPC Global Partners. Integrated Food Security Phase Classification Technical Manual Version 2.0. Evidence and Standards for Better Food Security Decisions. Rome: FAO-UN on behalf of IPC Global Partners, 2012. p 32.

8. NBC Domestic Preparedness Training Hospital Provider Course. Undated. Curriculum available from: Center for Domestic Preparedness, Anniston AL, http:// cdp.dhs.gov/. 


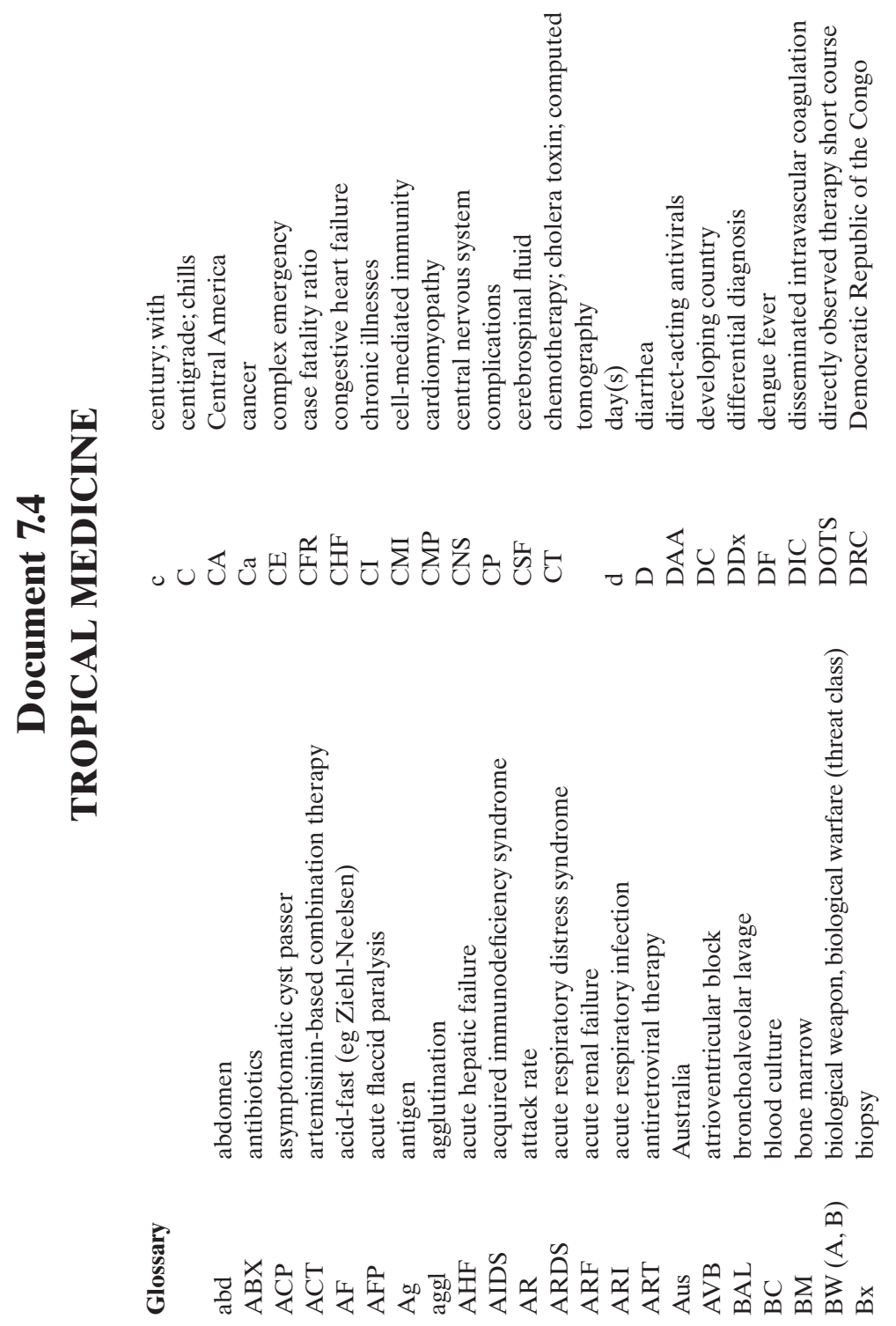




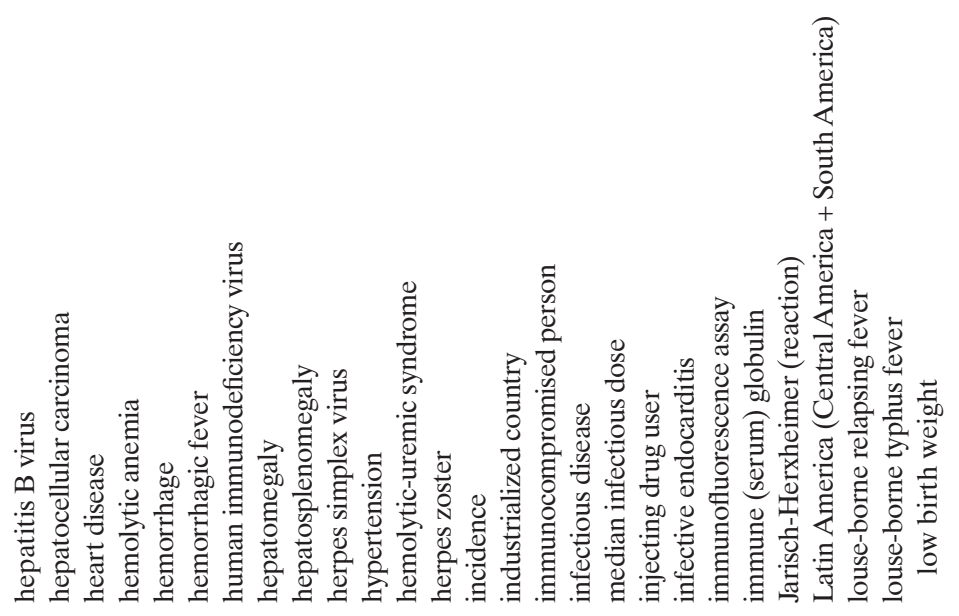

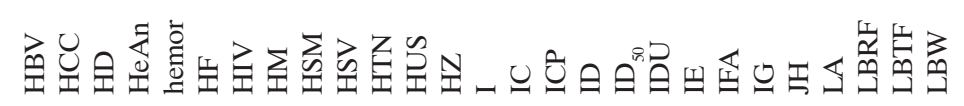

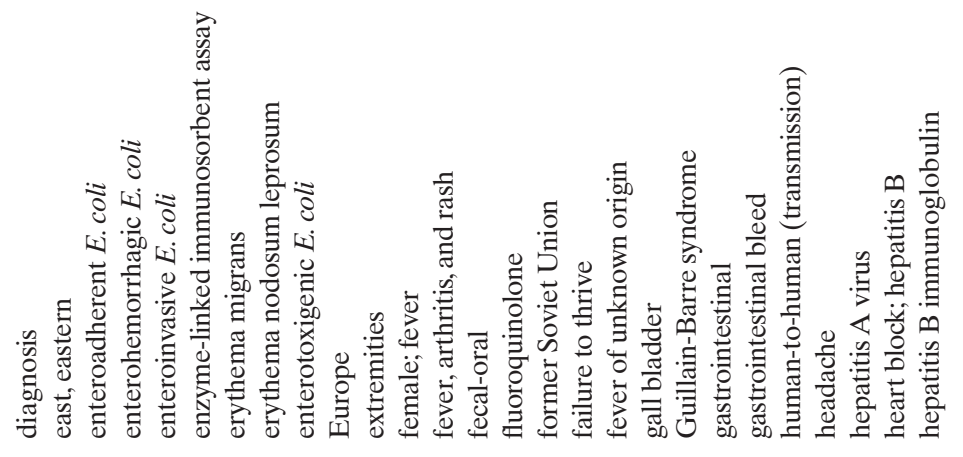

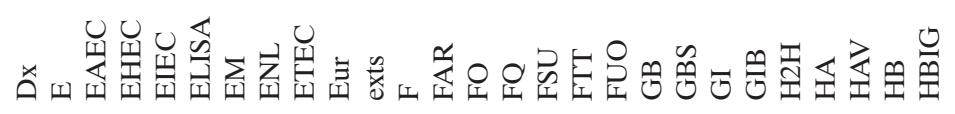




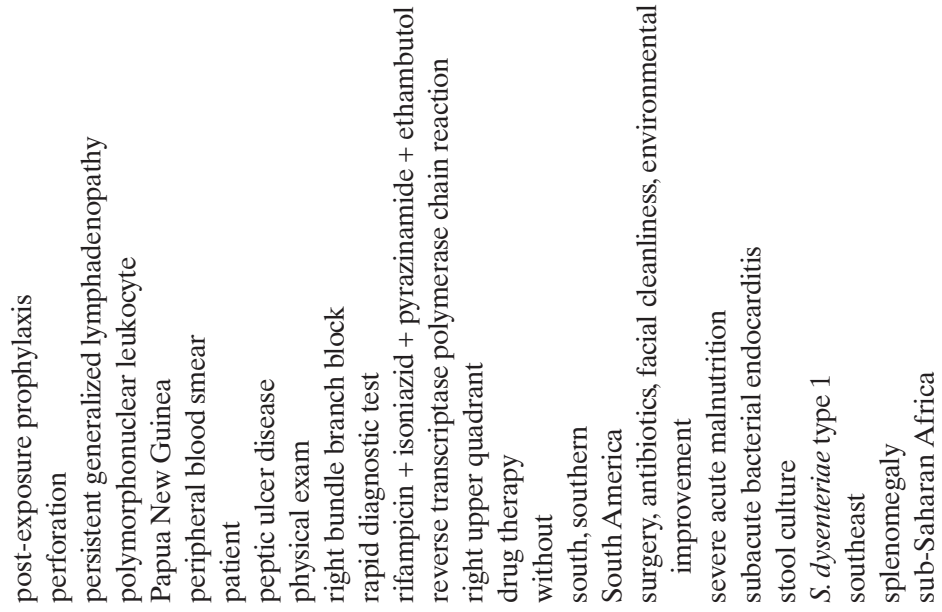

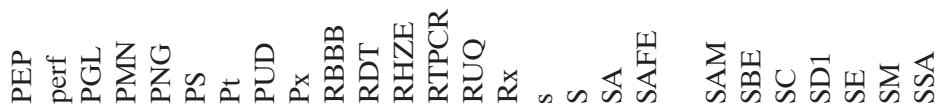

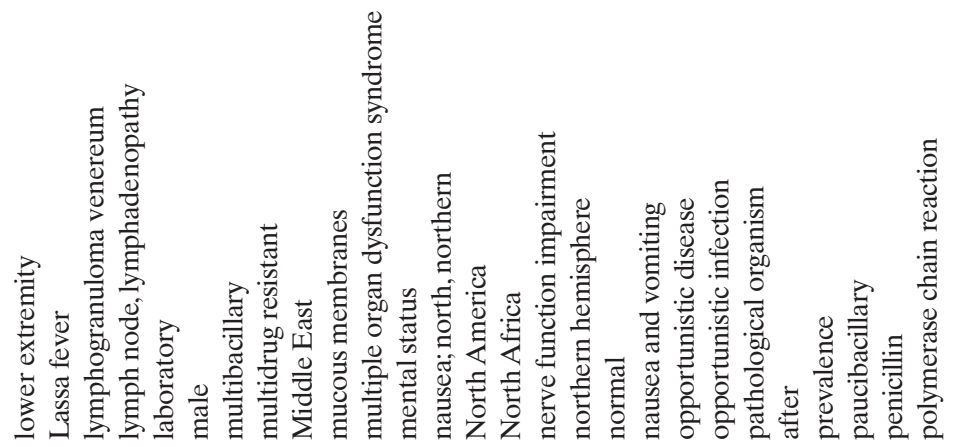

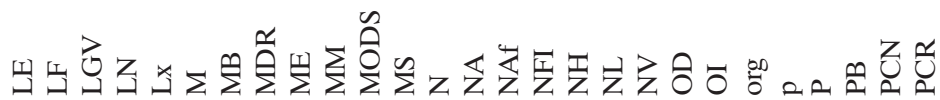




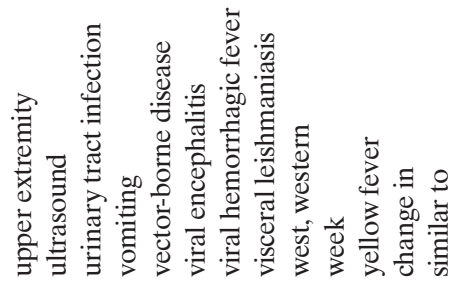

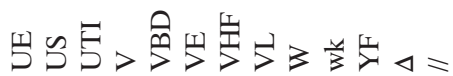

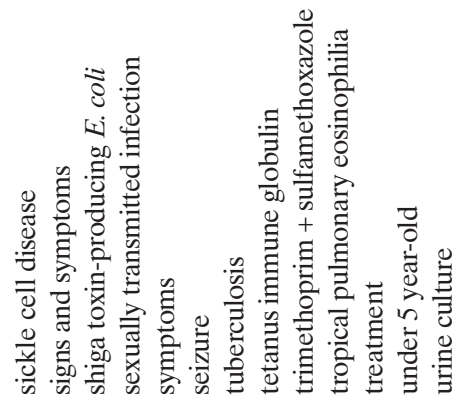

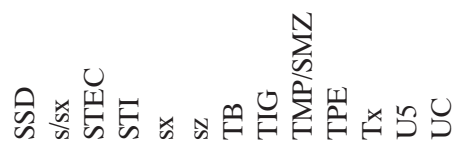

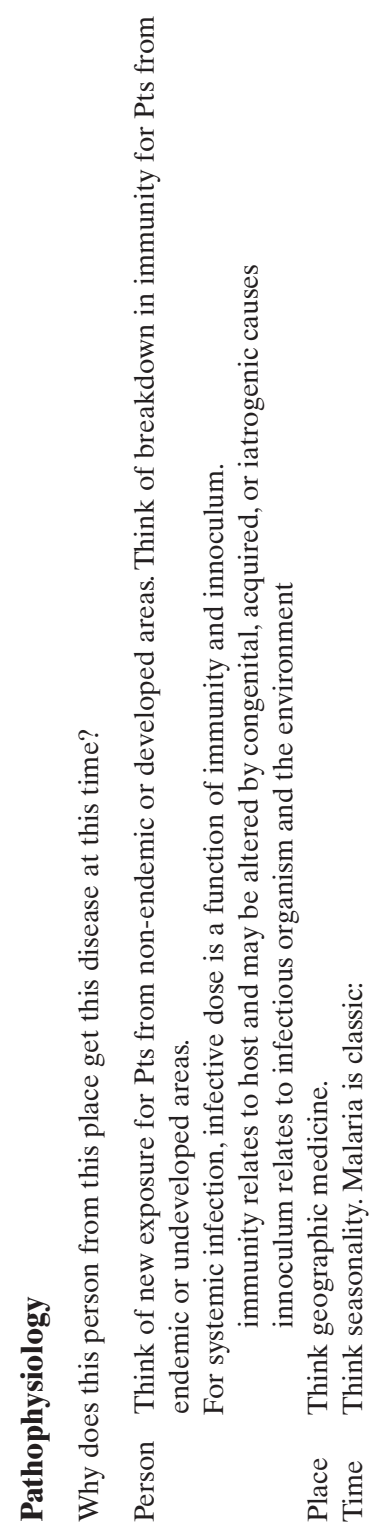


Document 7.4 191

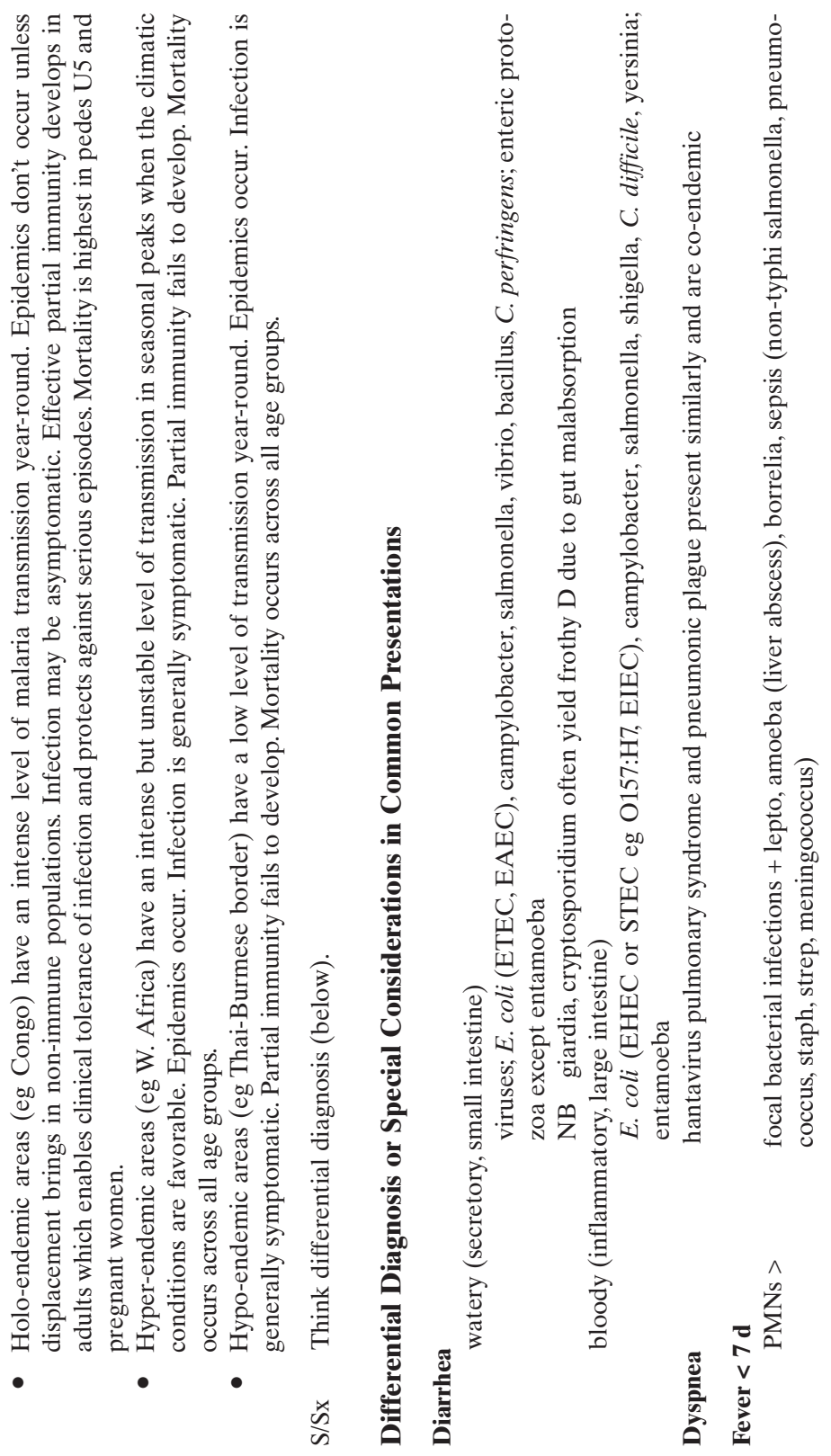




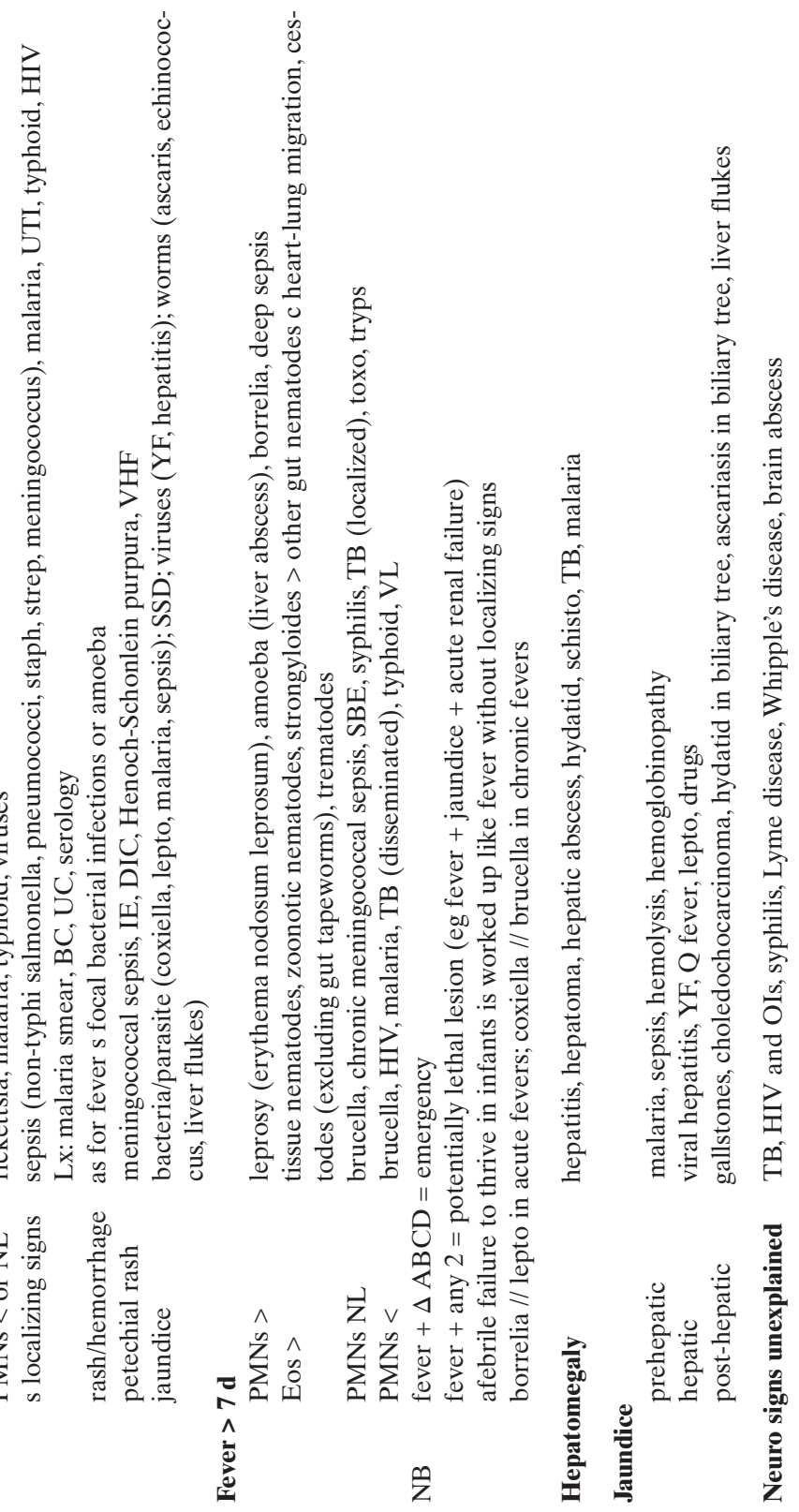




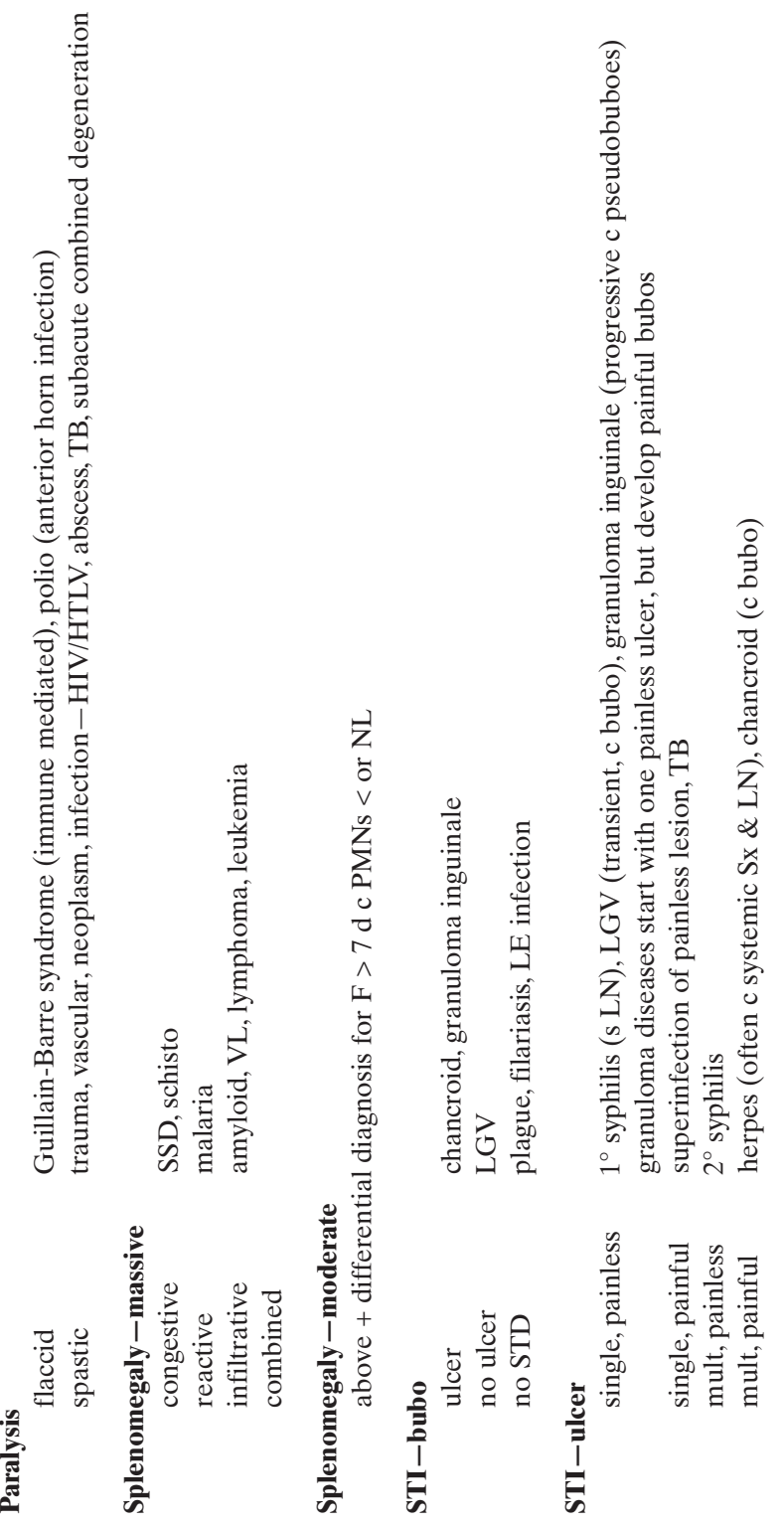


跑

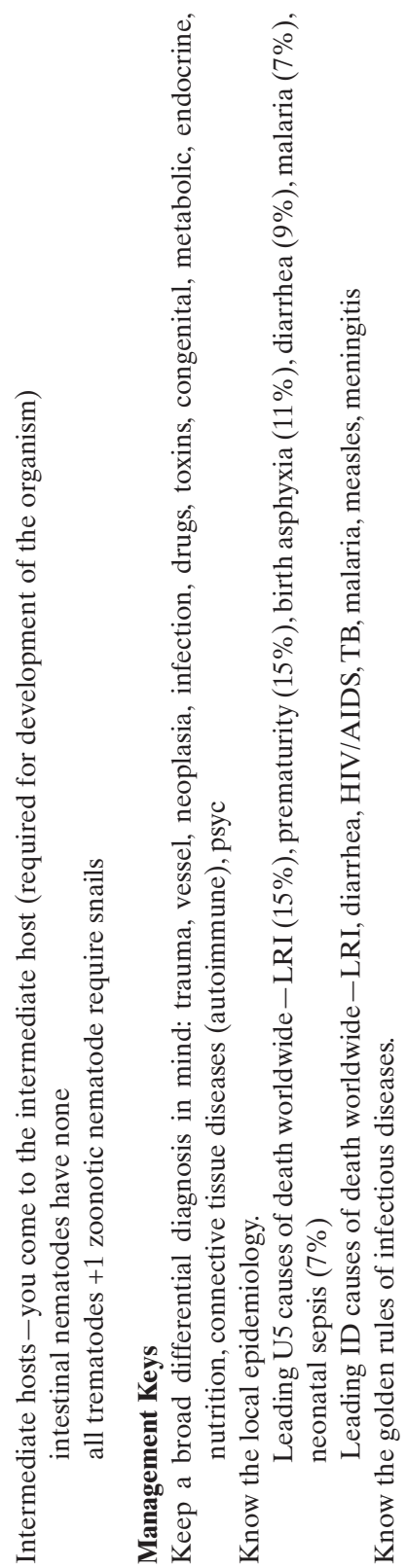


Document 7.4 195

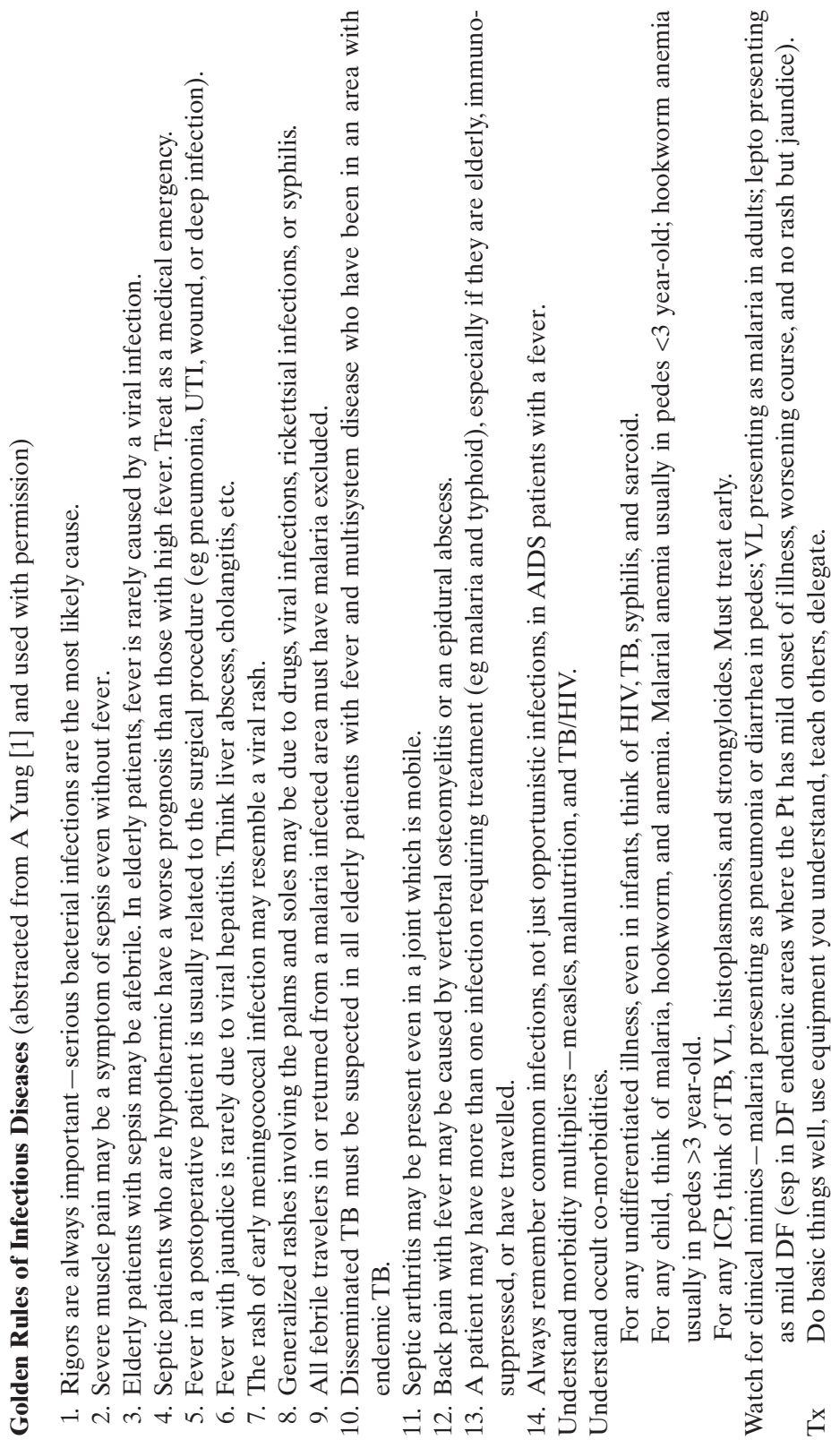




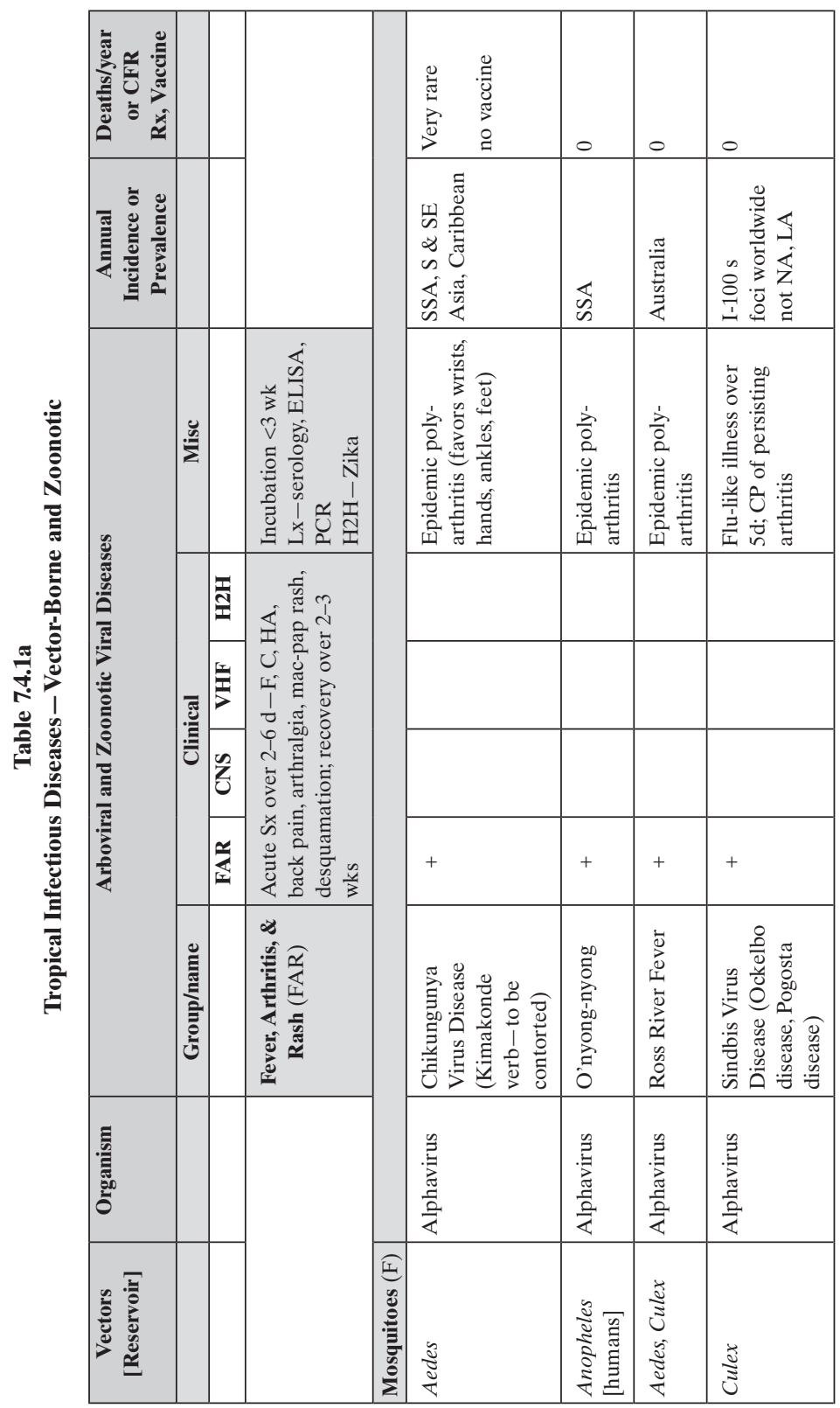


Document 7.4 197

\begin{tabular}{|c|c|c|c|c|}
\hline 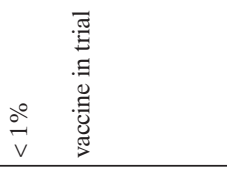 & 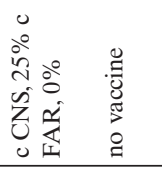 & 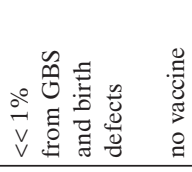 & & 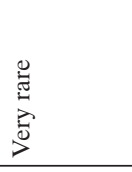 \\
\hline 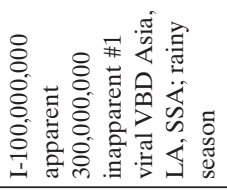 & 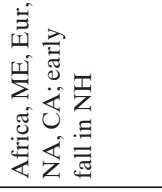 & 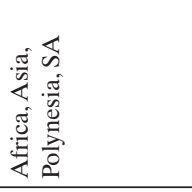 & & 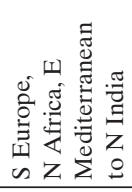 \\
\hline 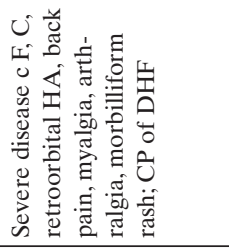 & 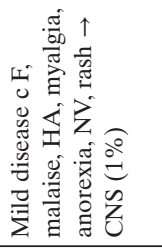 & 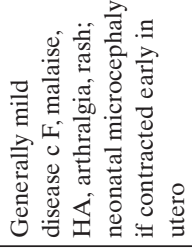 & & \\
\hline & & $+\stackrel{\vec{\theta}}{\underline{E}}$ & & \\
\hline+ & + & & & \\
\hline+ & + & + & & + \\
\hline 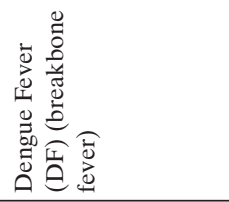 & 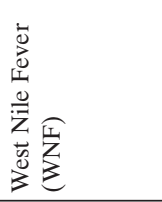 & 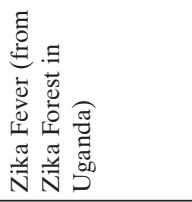 & & 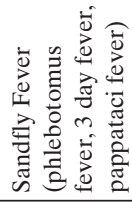 \\
\hline 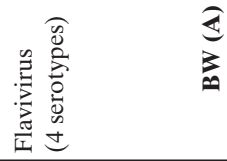 & $\sum_{\frac{\pi}{I}}^{\mathscr{Z}}$ & $\sum_{\substack{1\\
}}^{0}$ & & 疍 \\
\hline 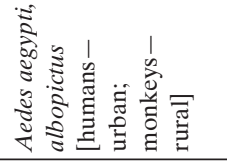 & $\underset{\widetilde{J}}{\stackrel{\Xi}{J ~}}$ & $\frac{\tilde{s}}{\tilde{D}}$ & 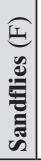 & 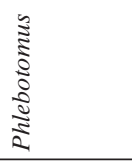 \\
\hline
\end{tabular}


7. Technical Annexes

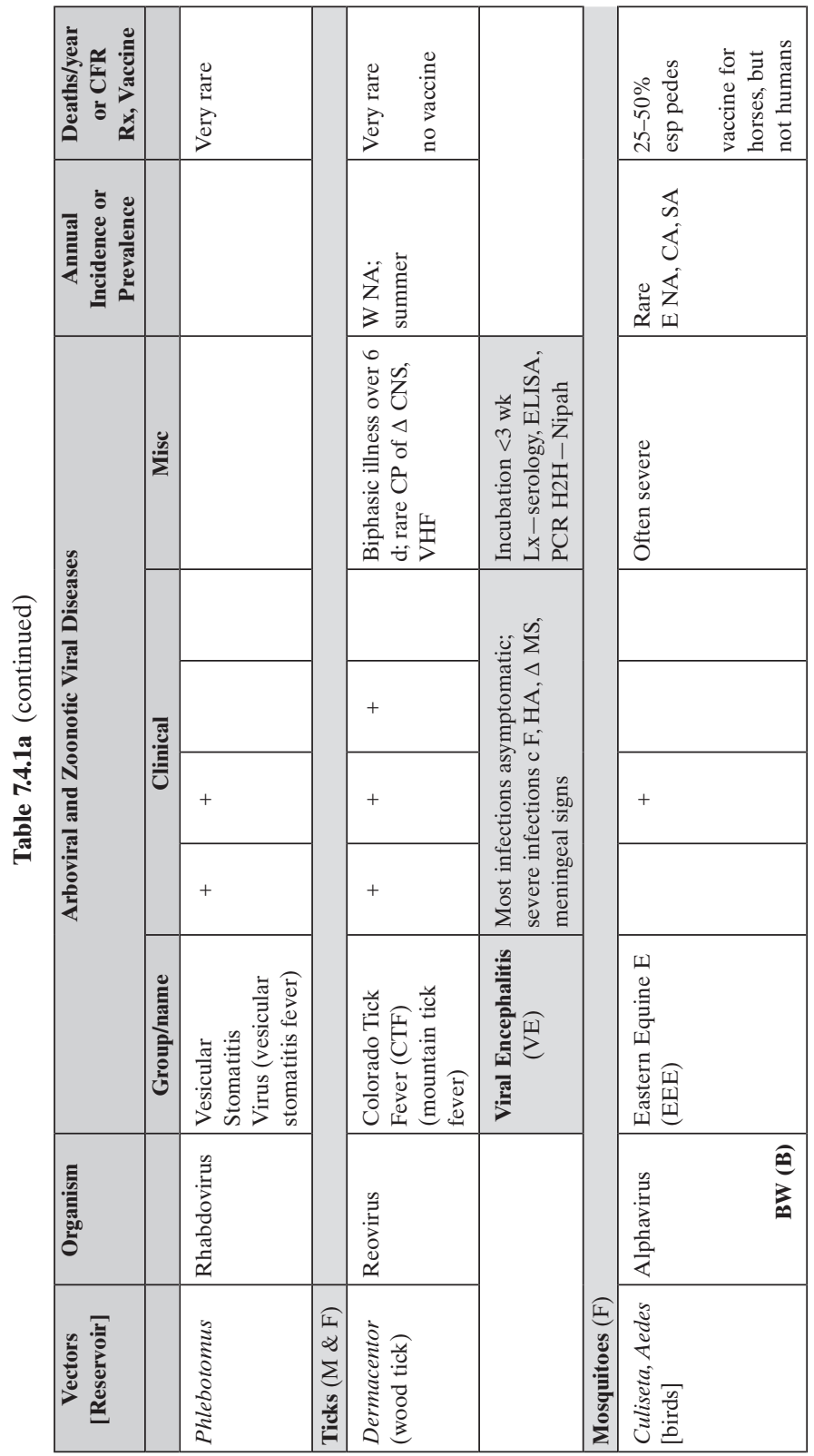


Document 7.4 199

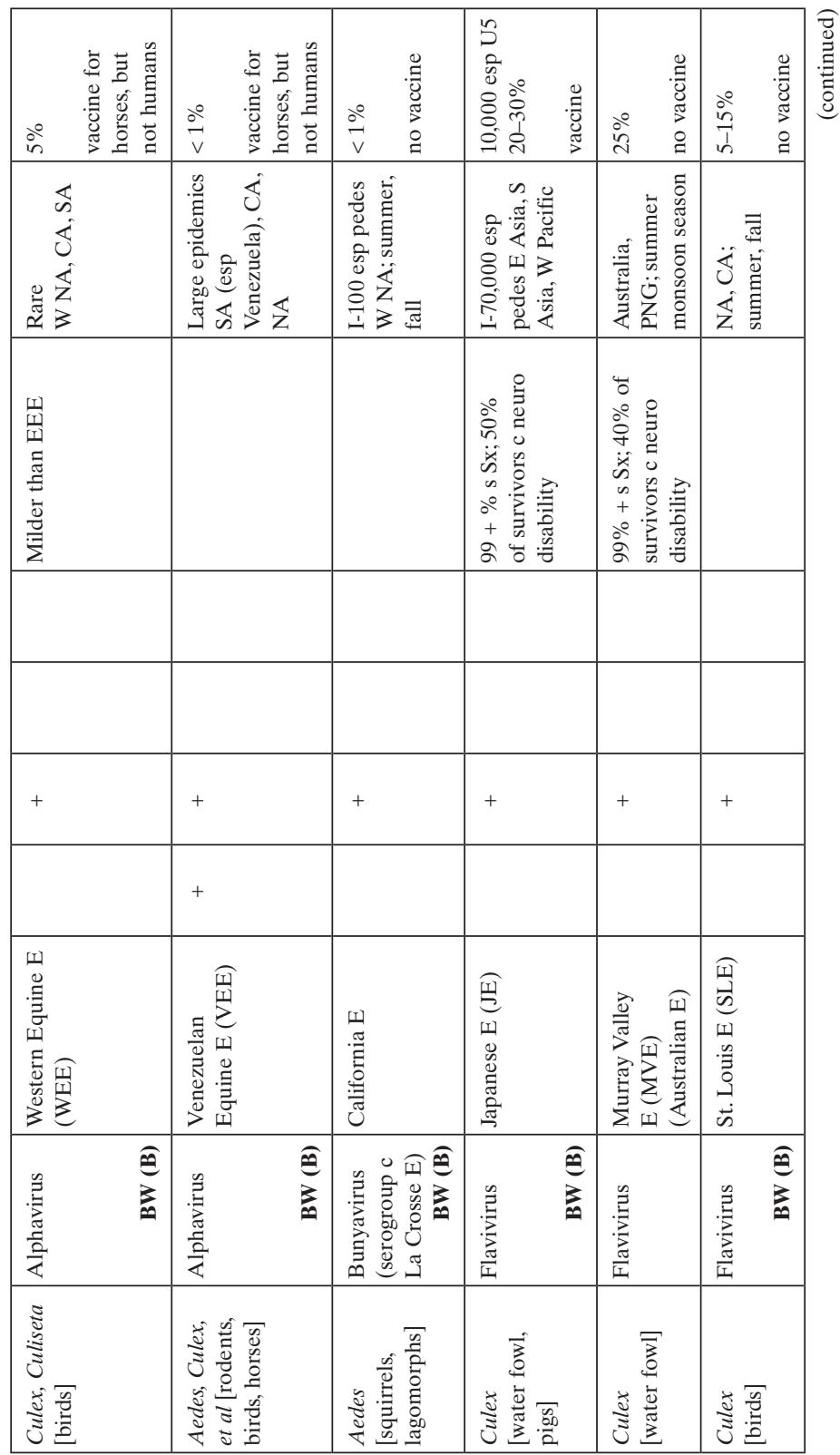


200 7. Technical Annexes

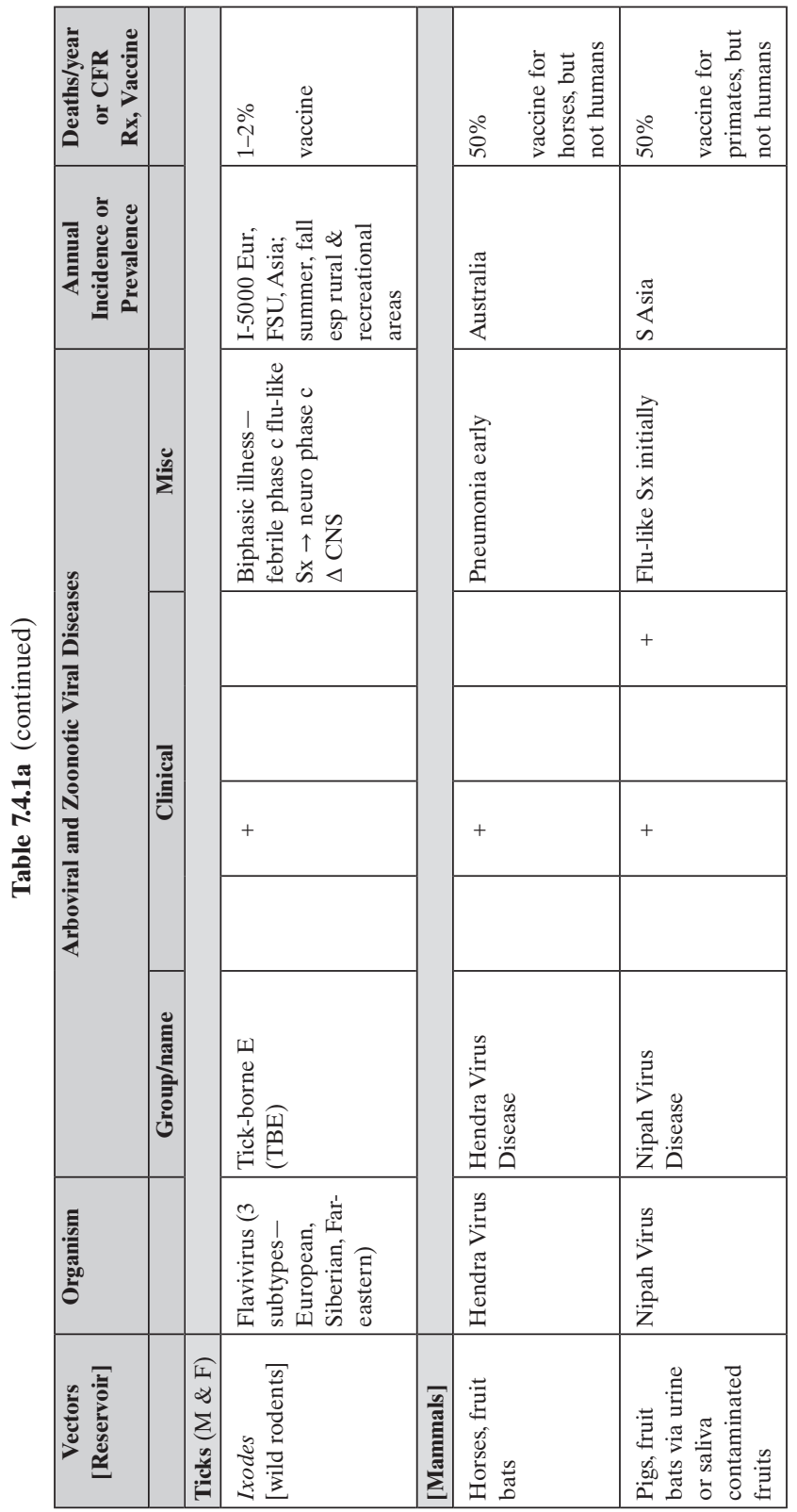


Document $7.4 \quad 201$

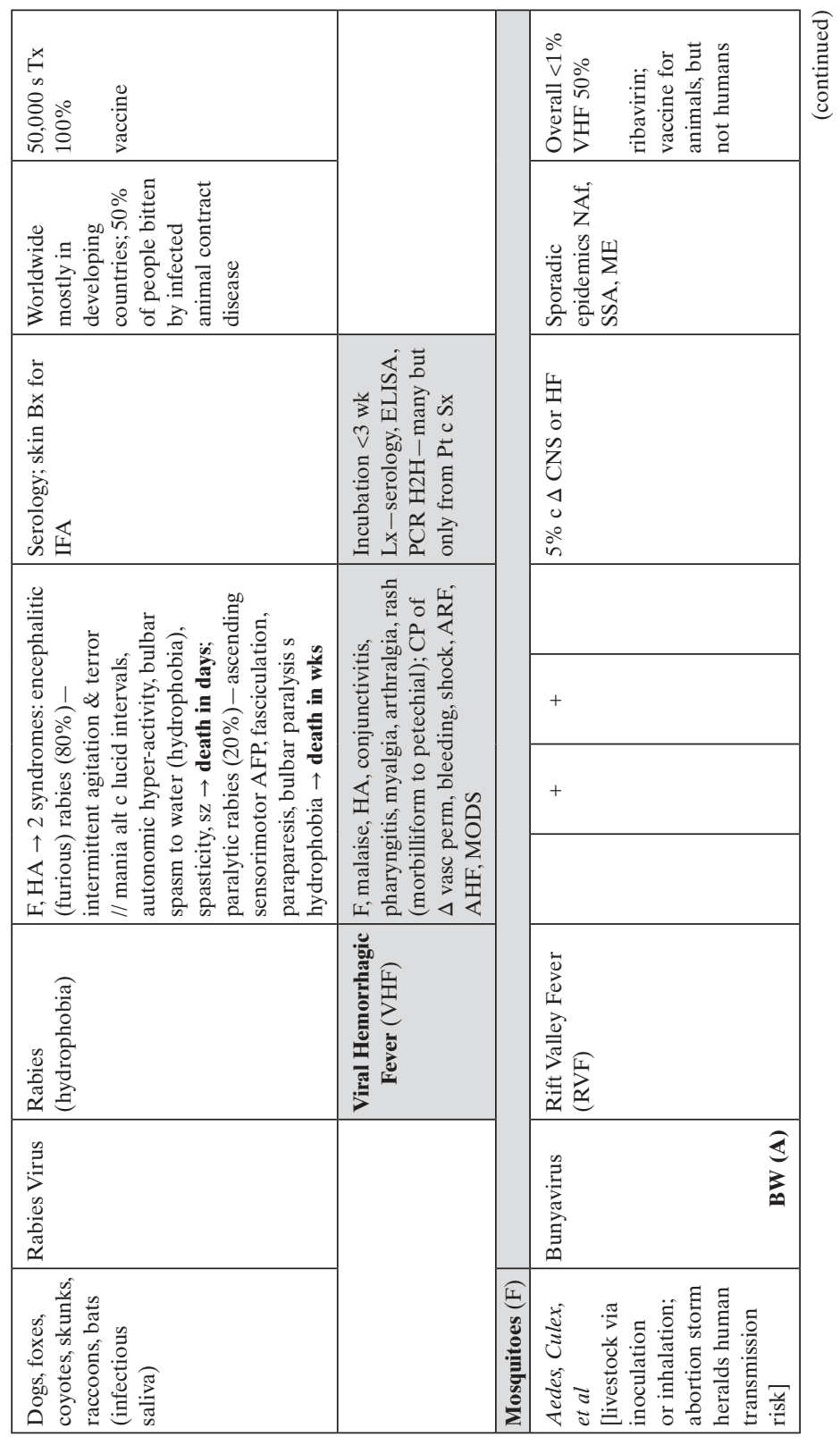




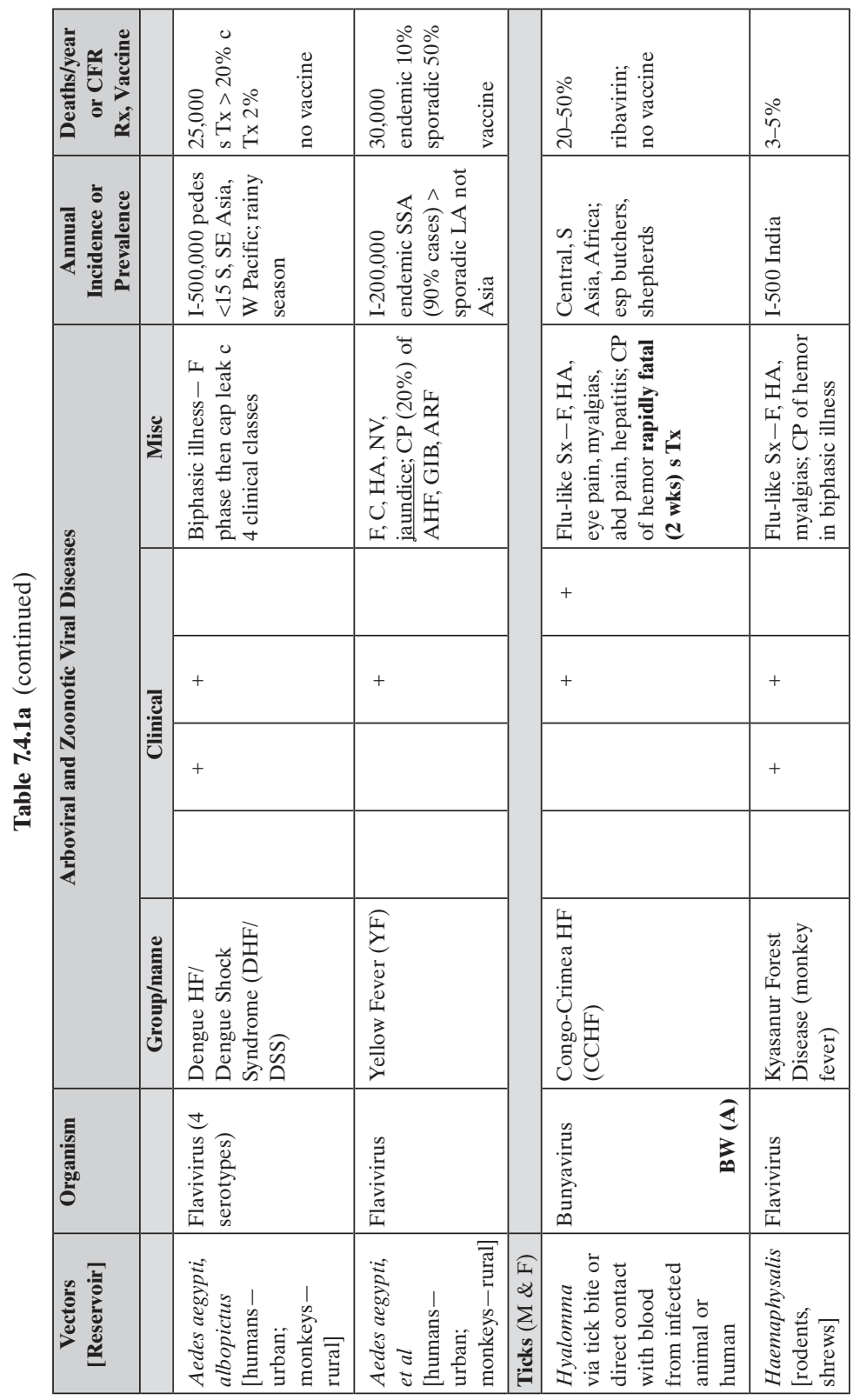


Document 7.4 203

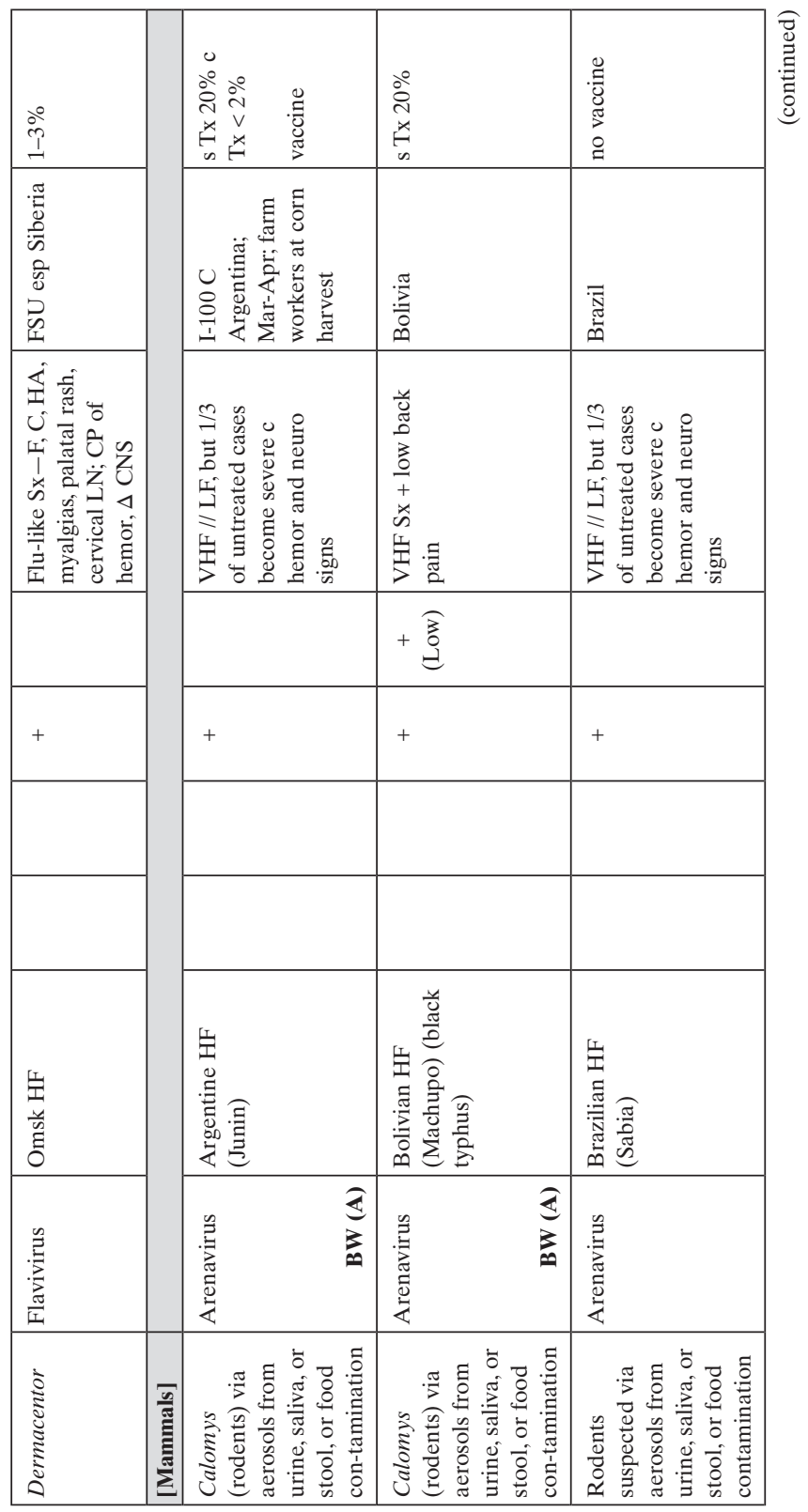


204 7. Technical Annexes

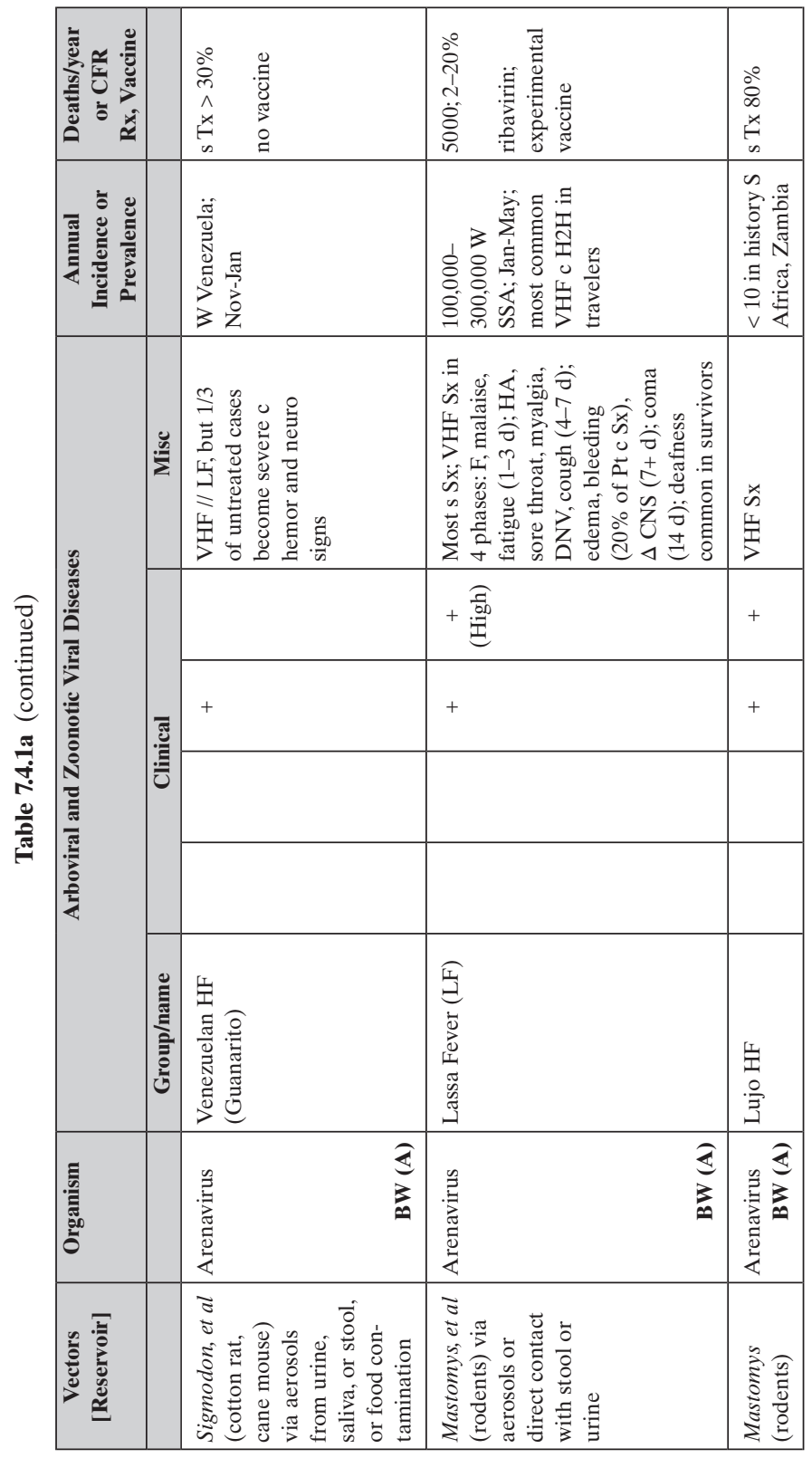


Document 7.4 205

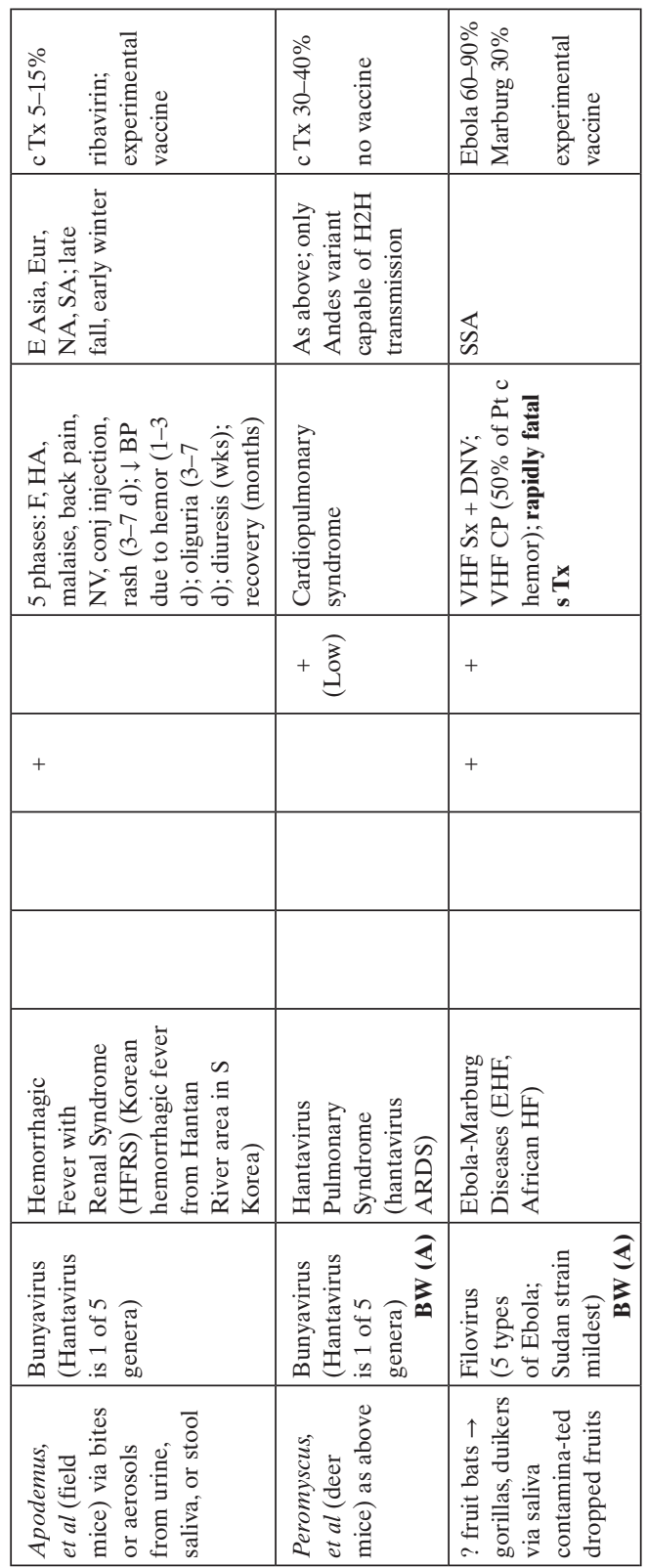


7. Technical Annexes

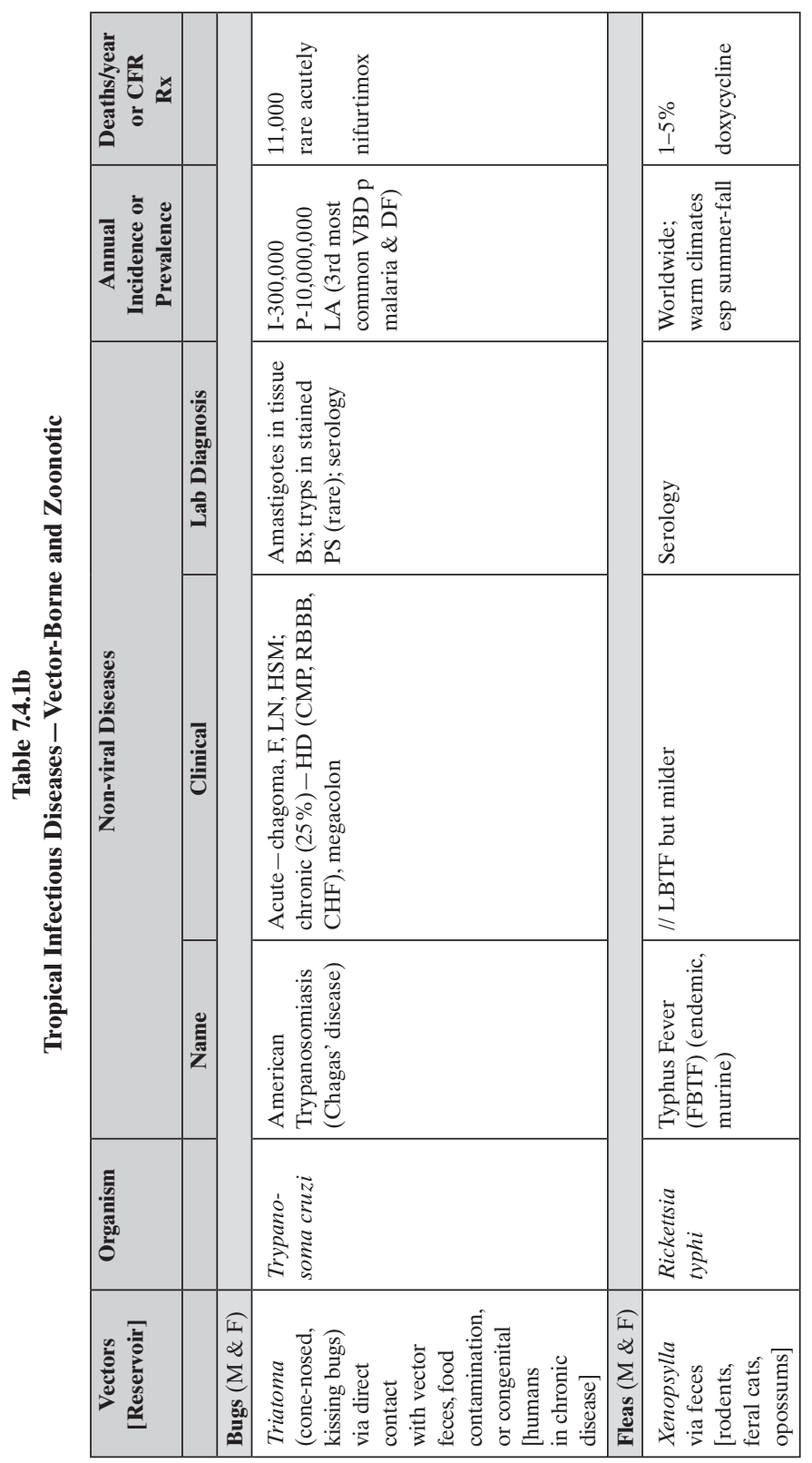


Document 7.4 207

\begin{tabular}{|c|c|c|}
\hline 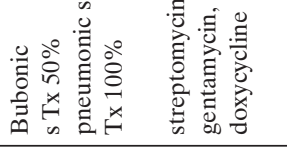 & 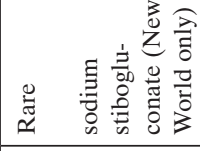 & 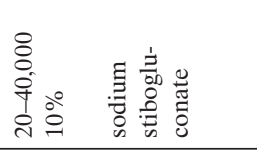 \\
\hline 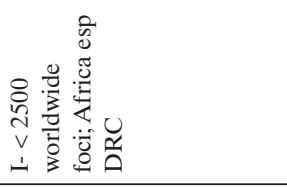 & 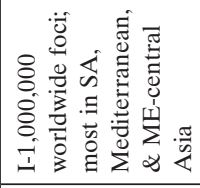 & 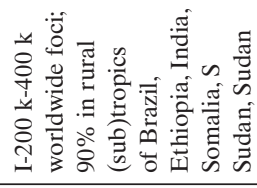 \\
\hline 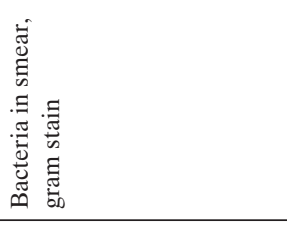 & 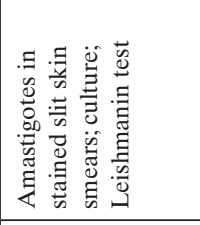 & 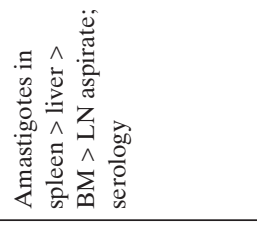 \\
\hline 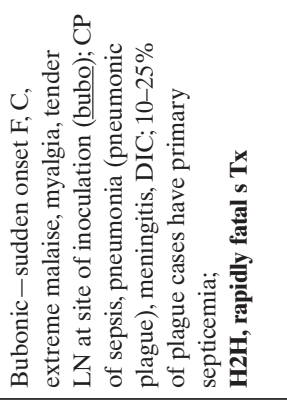 & 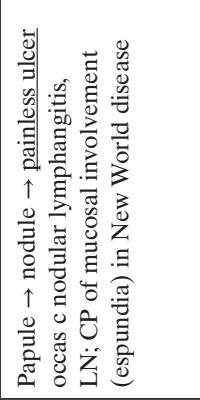 & 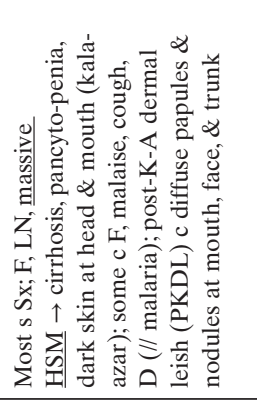 \\
\hline 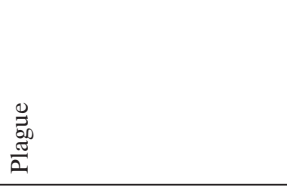 & 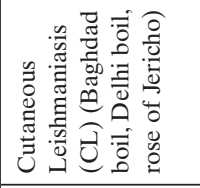 & 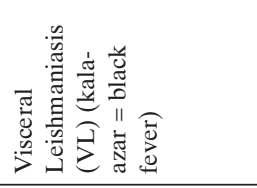 \\
\hline 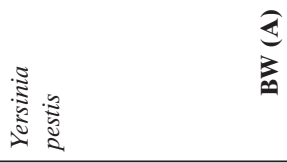 & 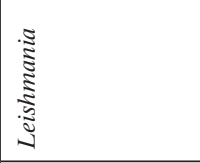 & 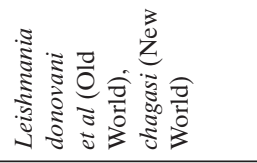 \\
\hline 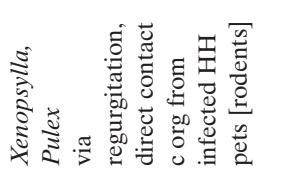 & 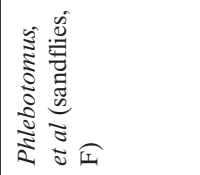 & 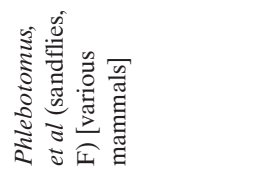 \\
\hline
\end{tabular}


7. Technical Annexes

\begin{tabular}{|c|c|c|c|c|c|}
\hline 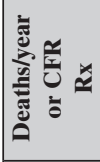 & & ○ 荘 & 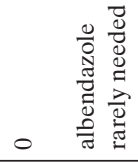 & 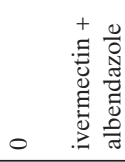 & 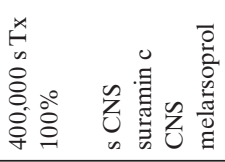 \\
\hline 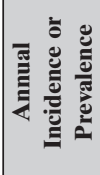 & & 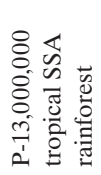 & 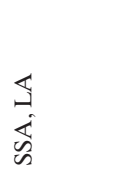 & 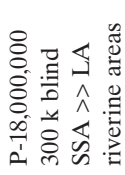 & 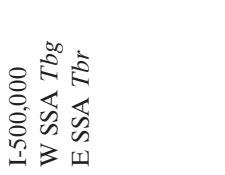 \\
\hline \multirow{3}{*}{ 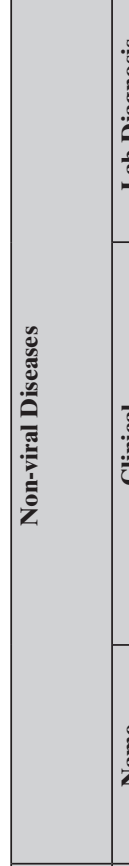 } & 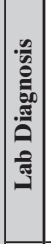 & 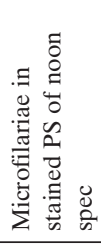 & 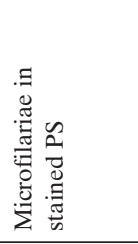 & 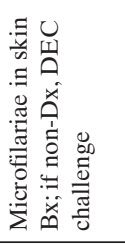 & 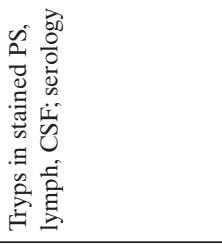 \\
\hline & (⿹ & 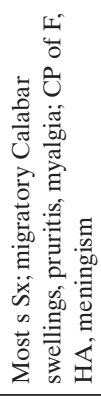 & 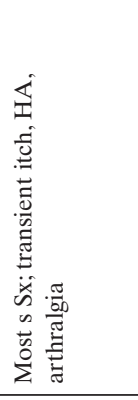 & 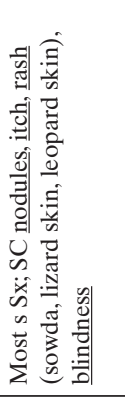 & 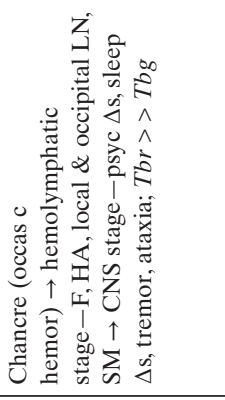 \\
\hline & 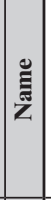 & 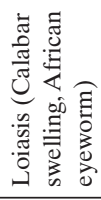 & 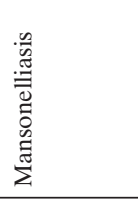 & 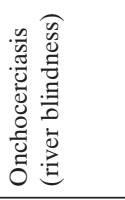 & 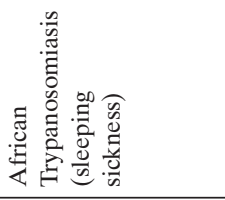 \\
\hline 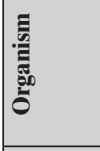 & & $\begin{array}{l}\tilde{s} \\
\tilde{s} \\
j\end{array}$ & 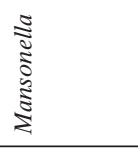 & 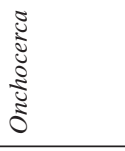 & 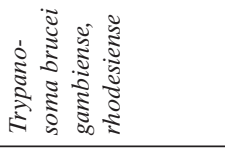 \\
\hline 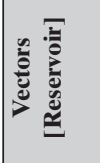 & & 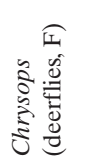 & 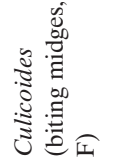 & 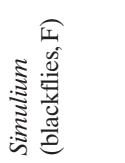 & 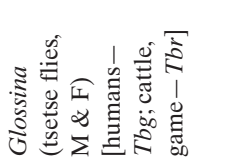 \\
\hline
\end{tabular}


Document 7.4 209

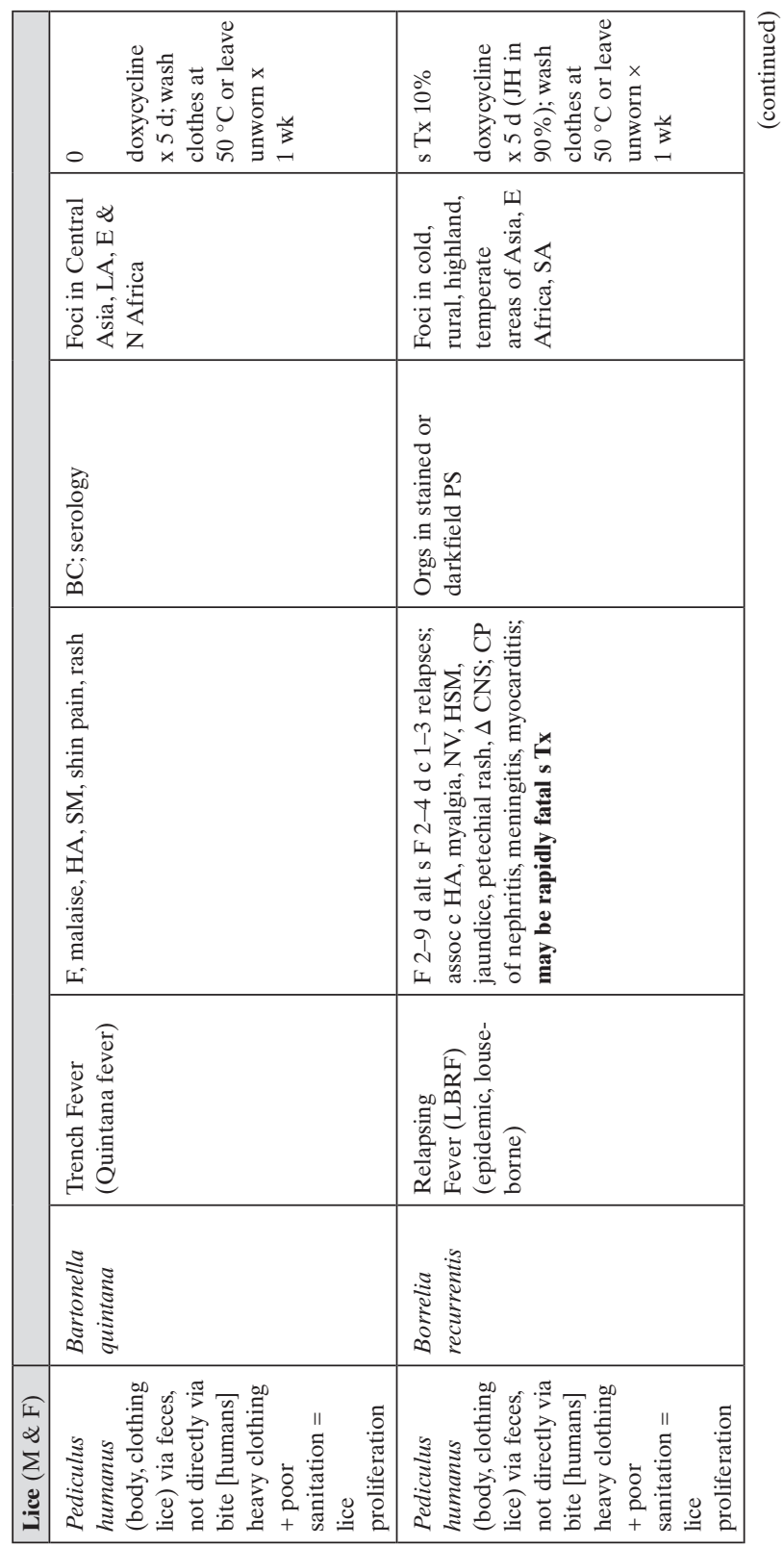




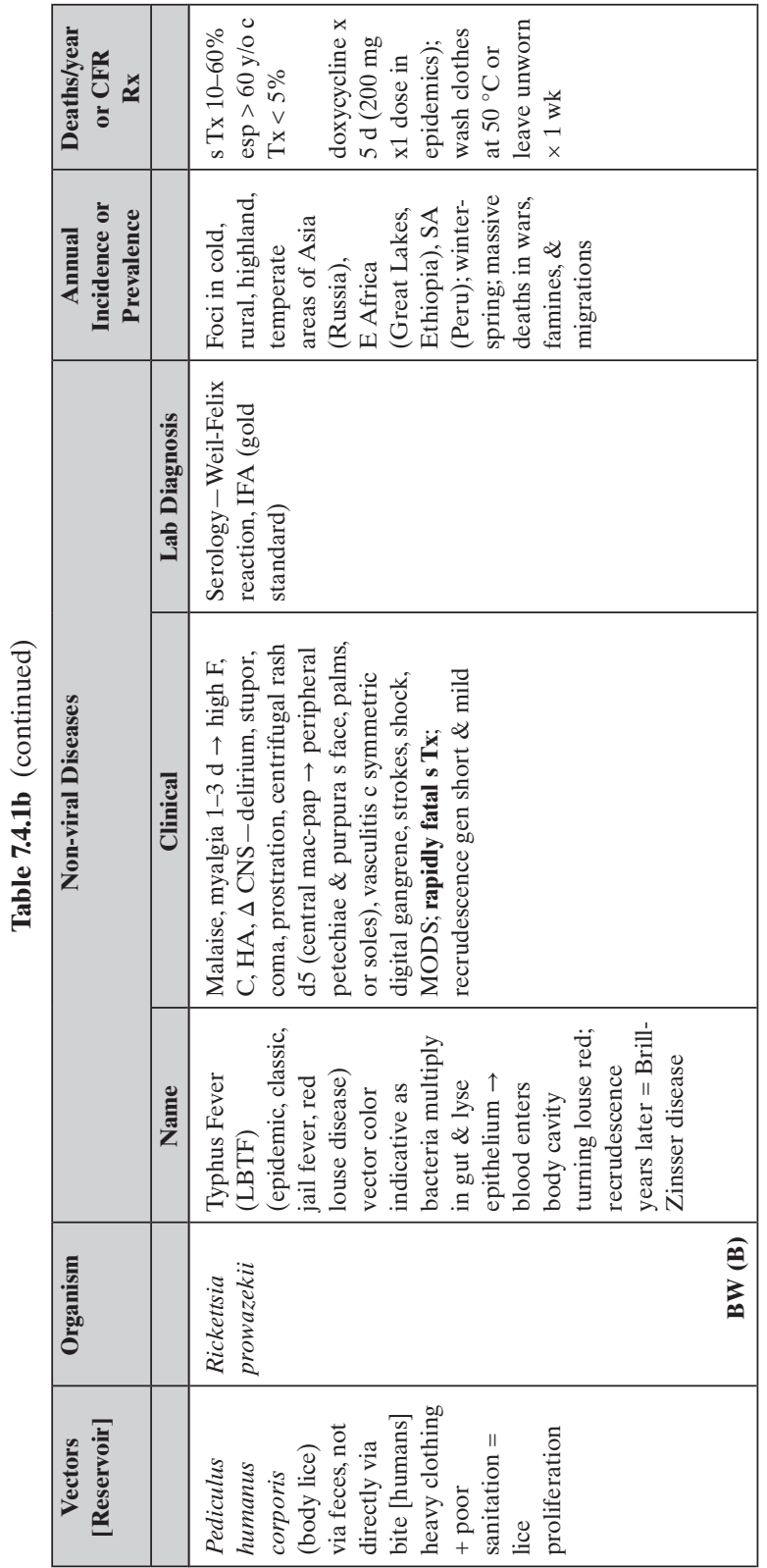


Document 7.4 211

\begin{tabular}{|c|c|c|c|c|c|}
\hline & 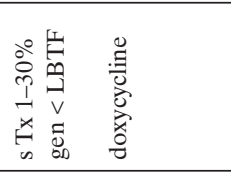 & 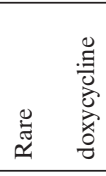 & & 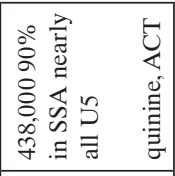 & 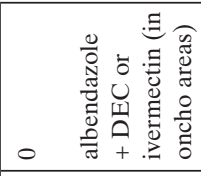 \\
\hline & 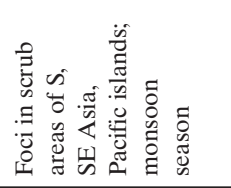 & 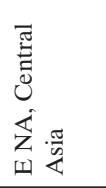 & & 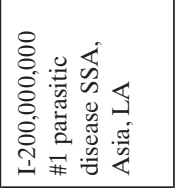 & 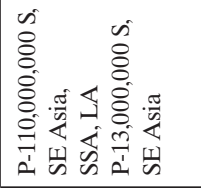 \\
\hline & 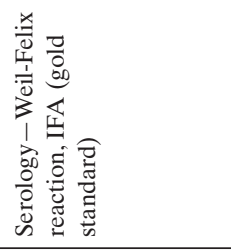 & $\begin{array}{l}\overrightarrow{c 0} \\
\frac{0}{0} \\
\vec{D} \\
\qquad \\
\qquad\end{array}$ & & 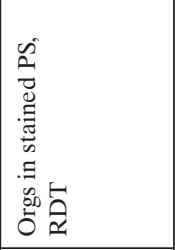 & 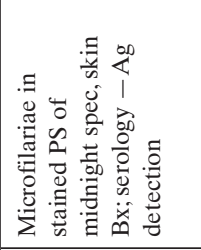 \\
\hline & 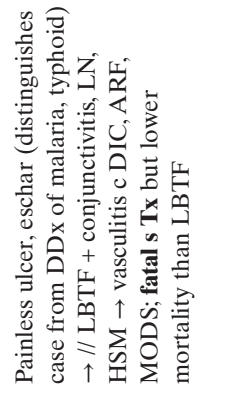 & 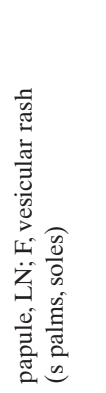 & & 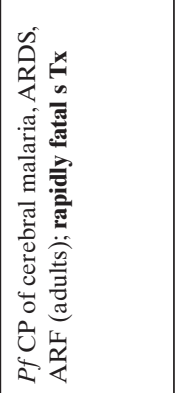 & 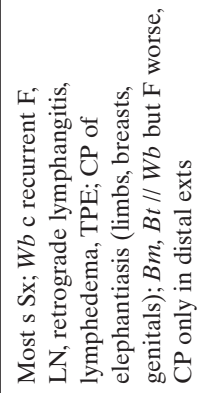 \\
\hline & 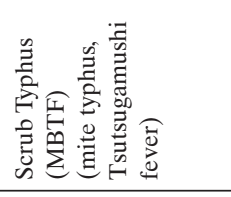 & 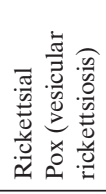 & & 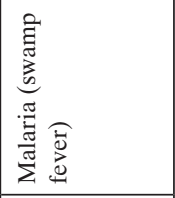 & 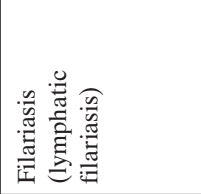 \\
\hline & 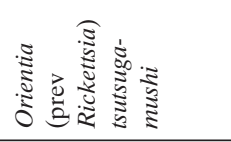 & 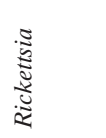 & & 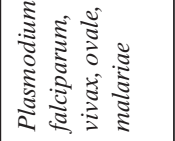 & 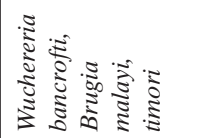 \\
\hline 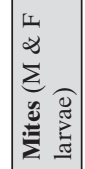 & 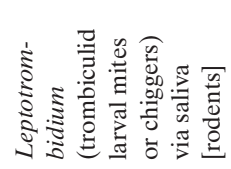 & 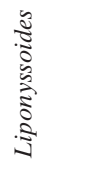 & 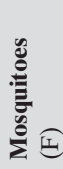 & 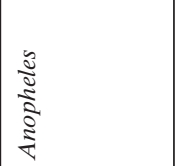 & 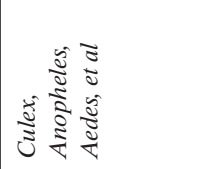 \\
\hline
\end{tabular}




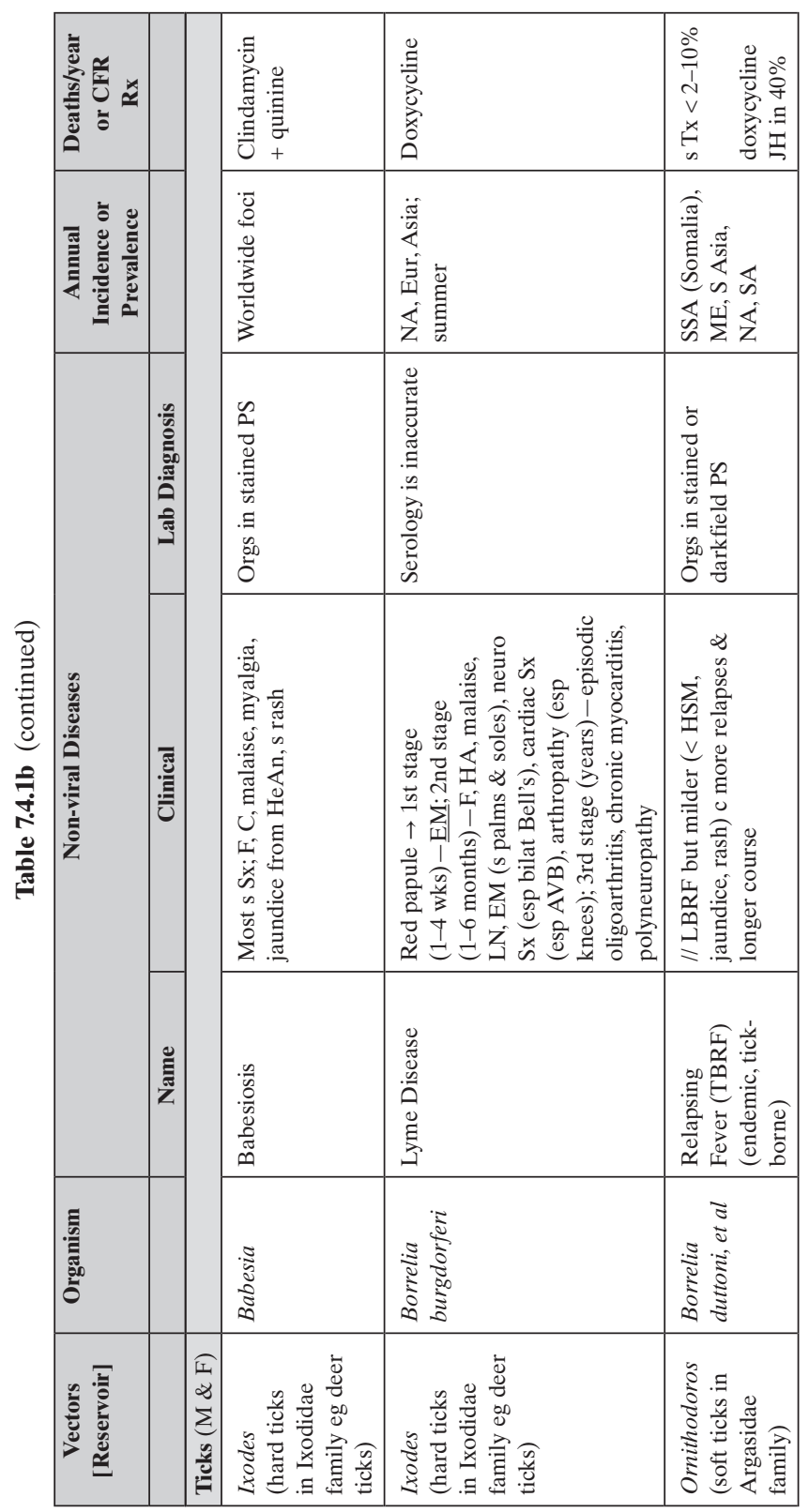


Document 7.4 213

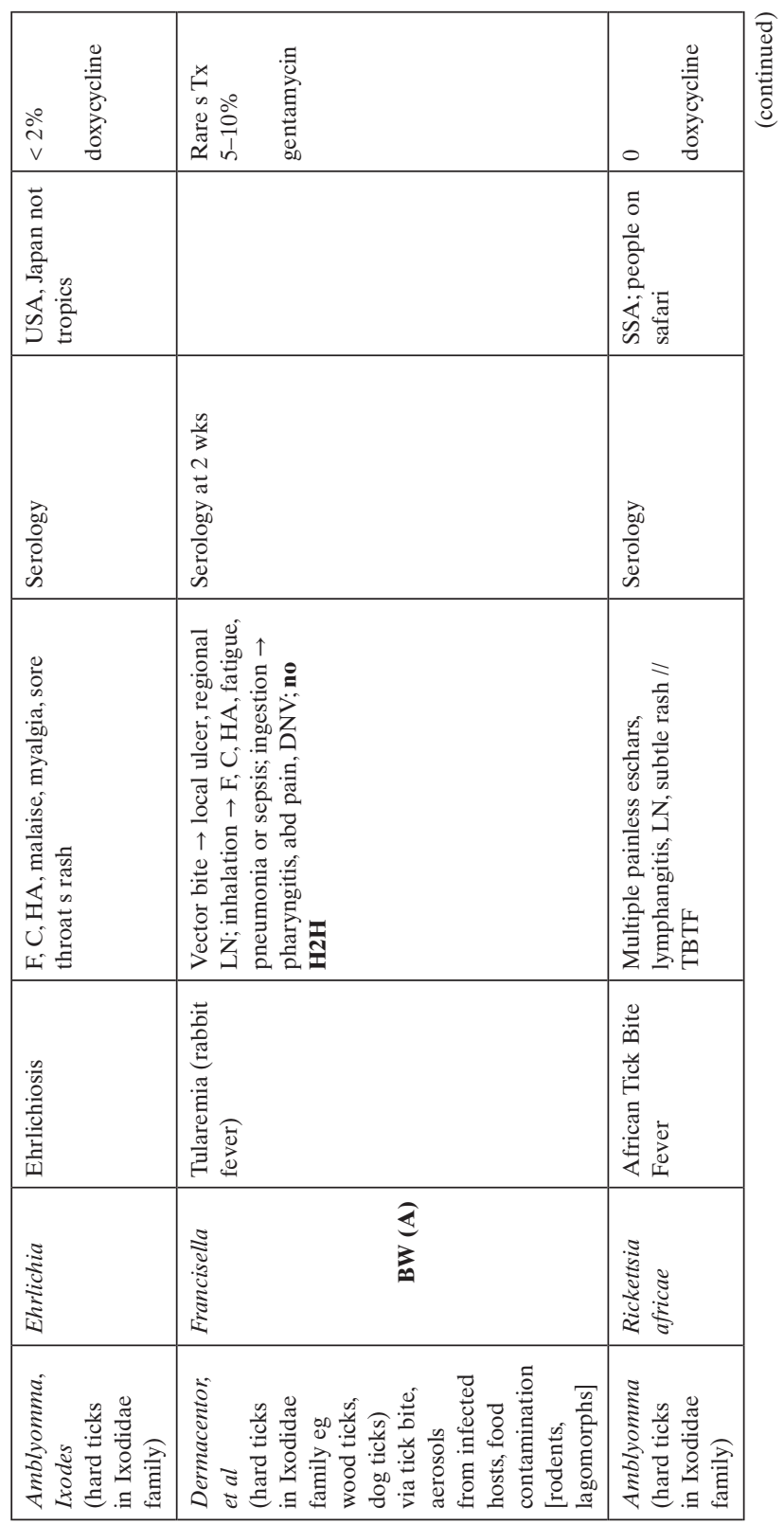


214 7. Technical Annexes

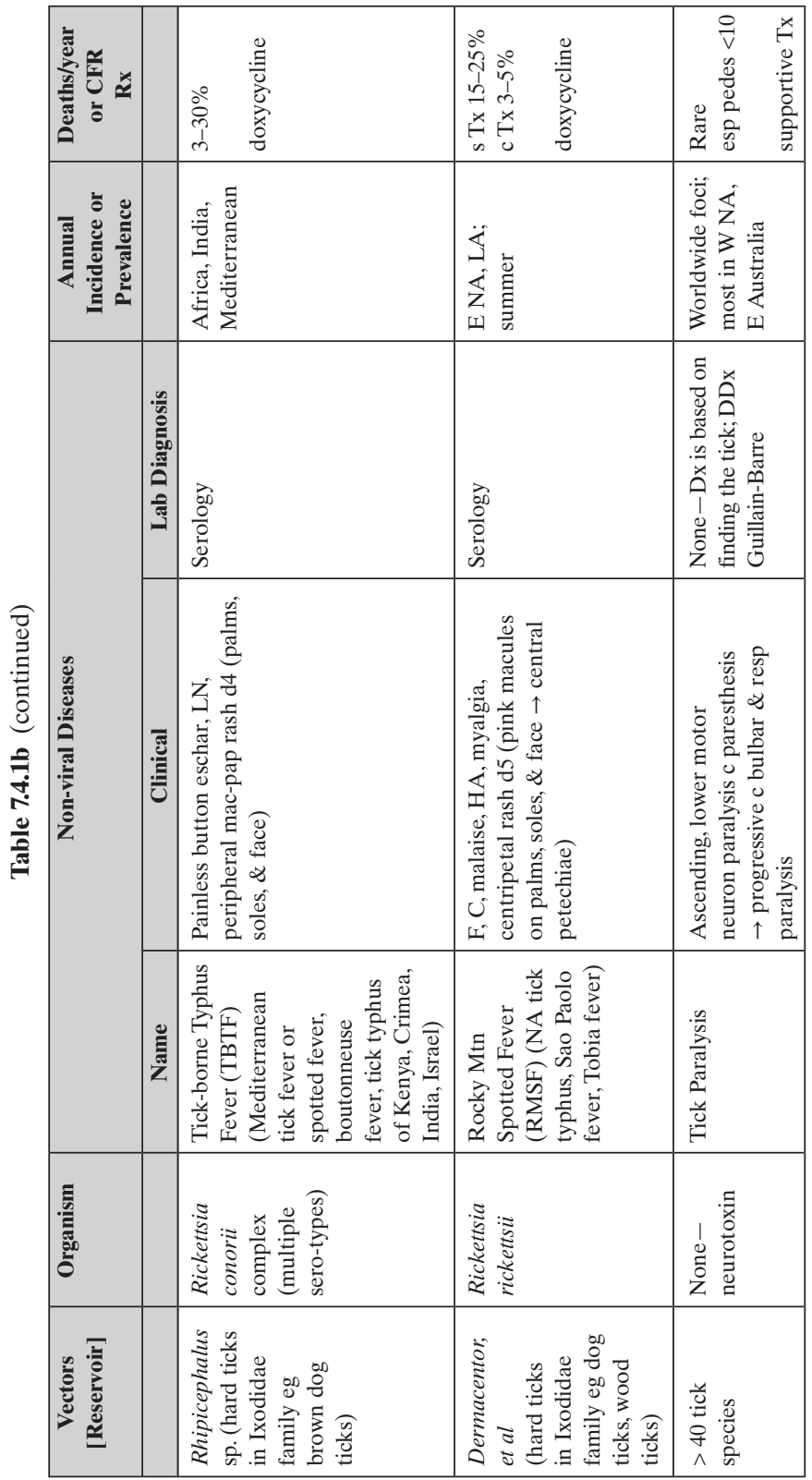


Document 7.4 215

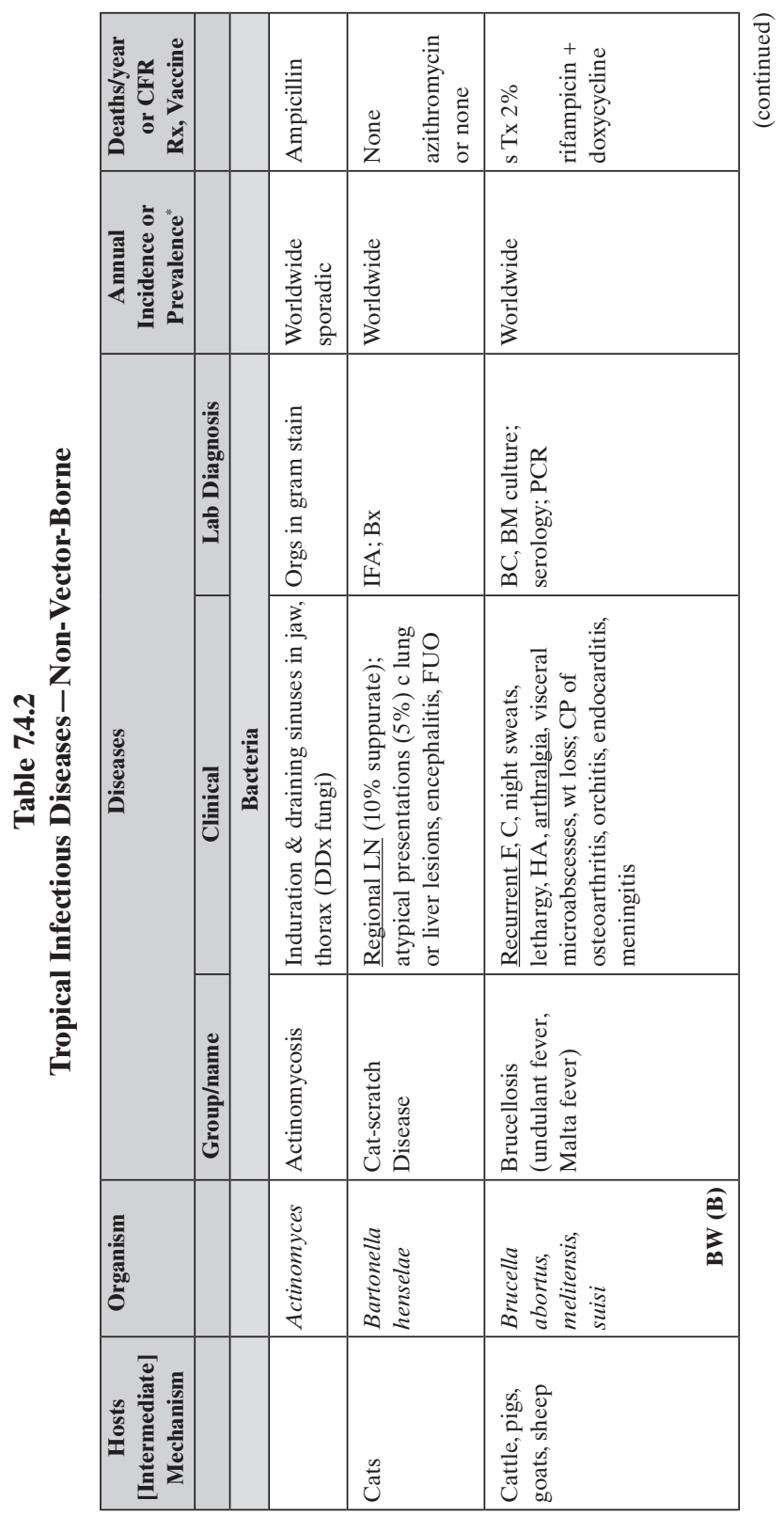




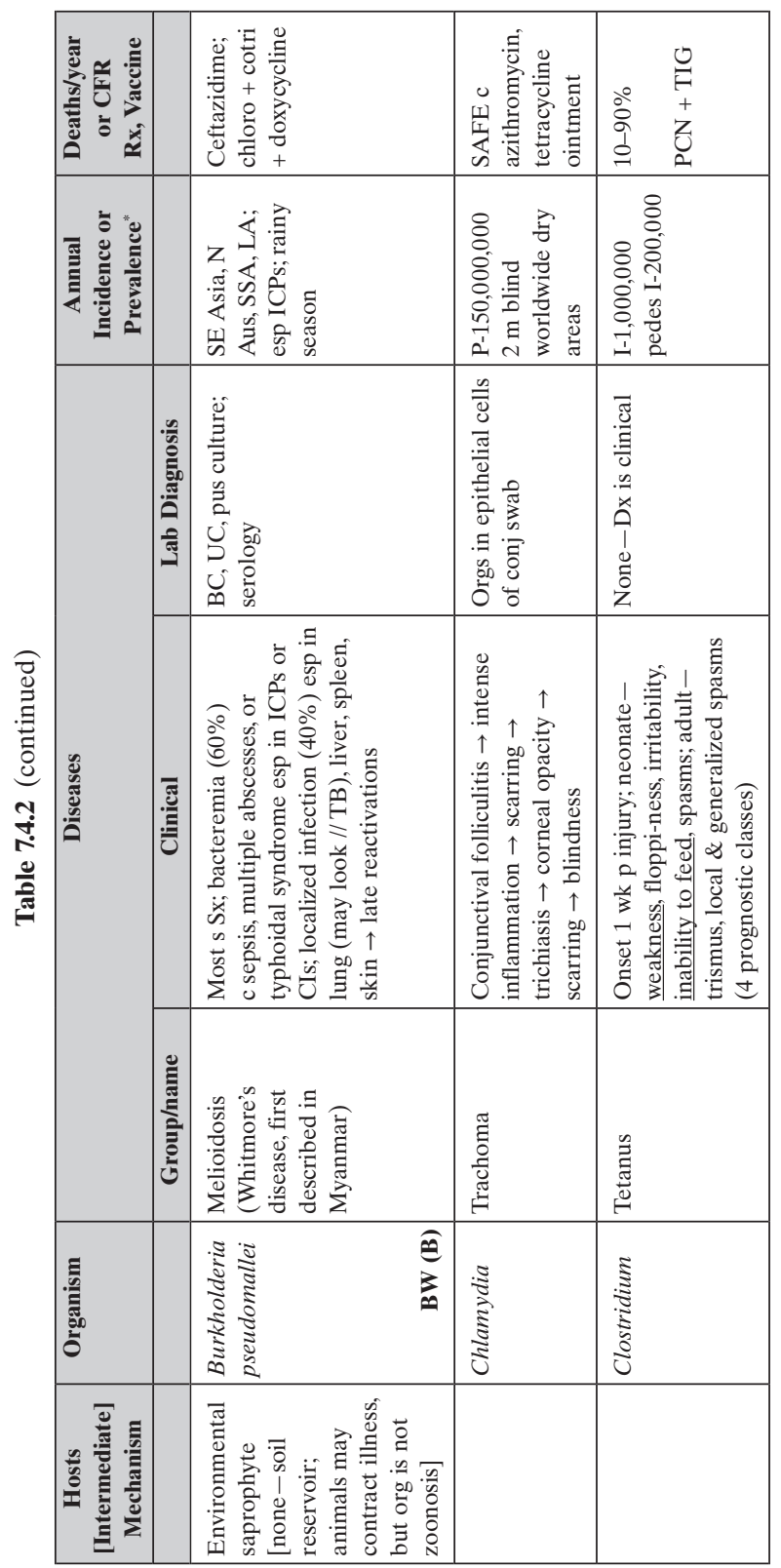


Document 7.4 217

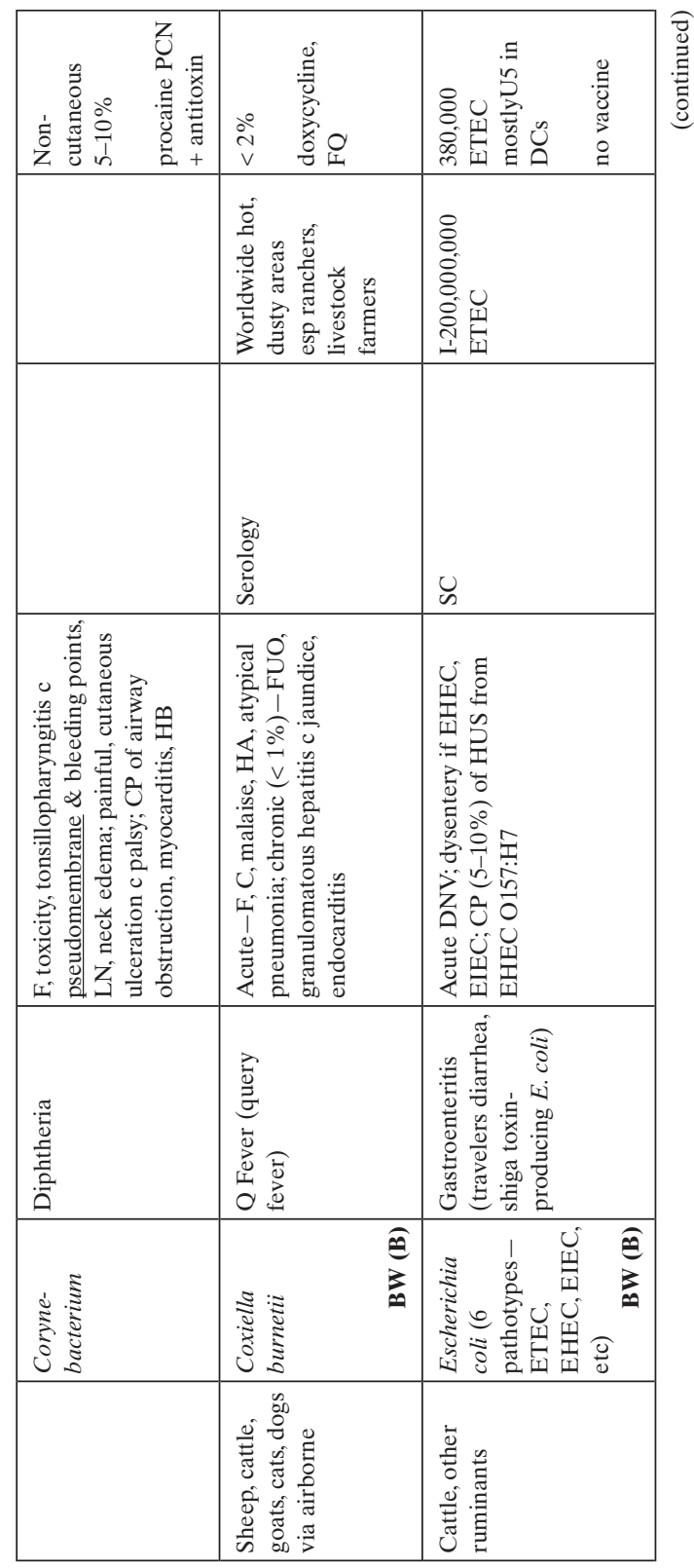




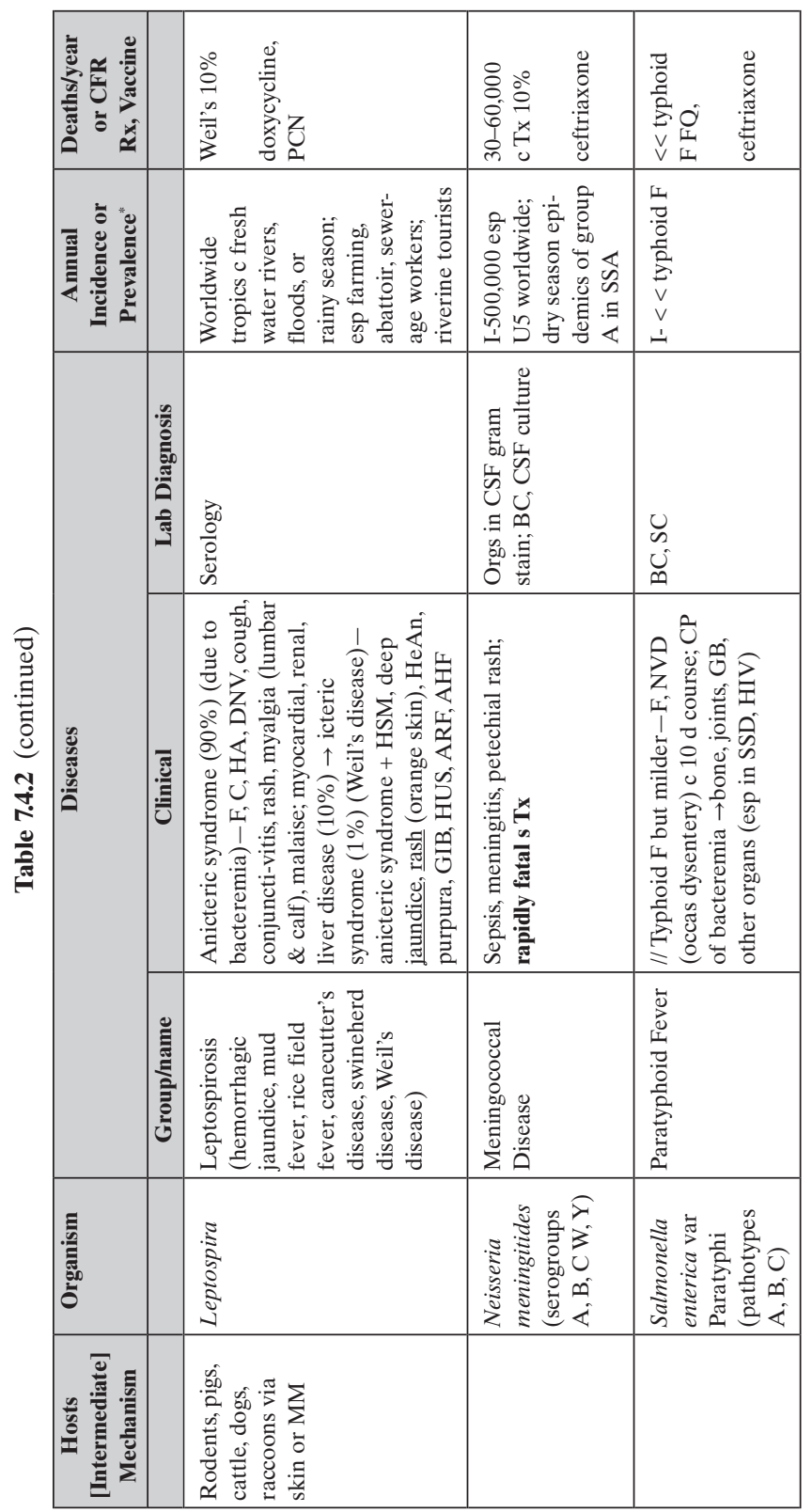


Document 7.4 219

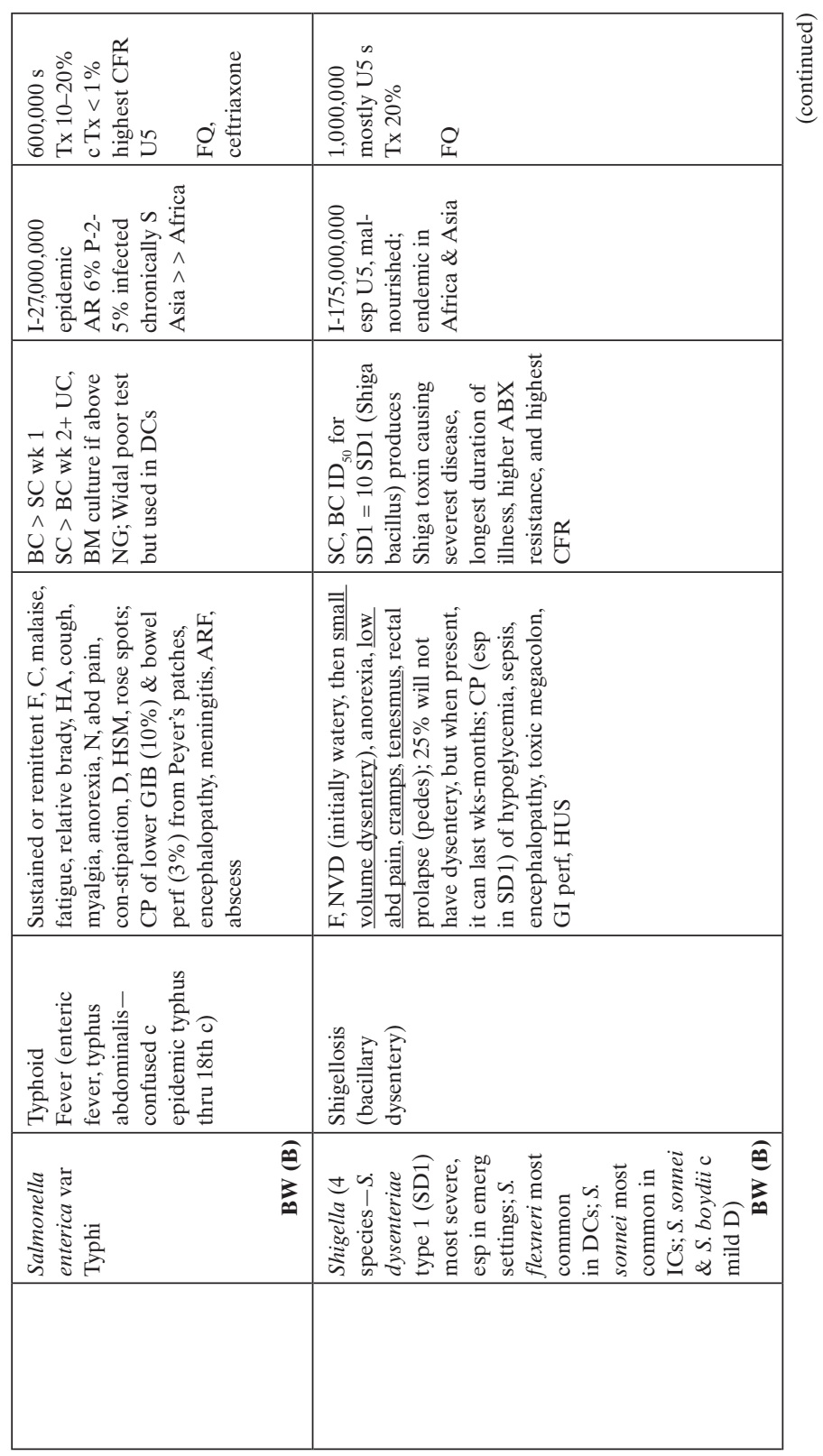




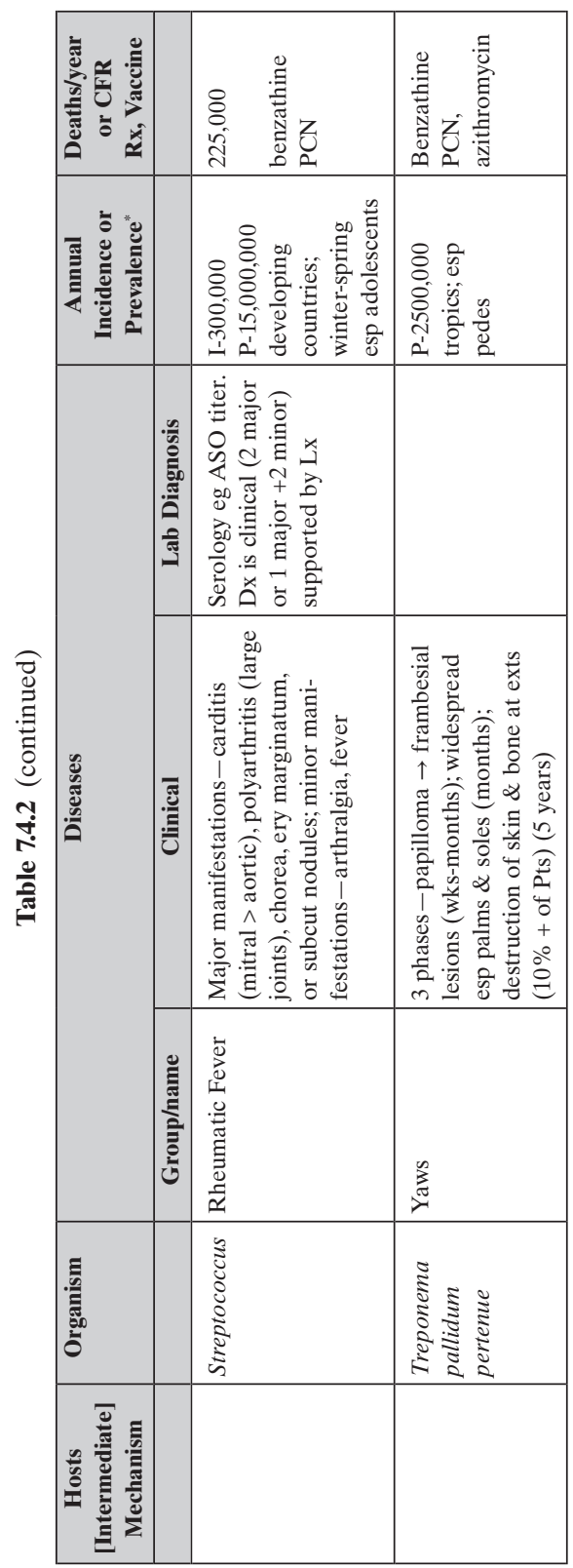


Document 7.4 221

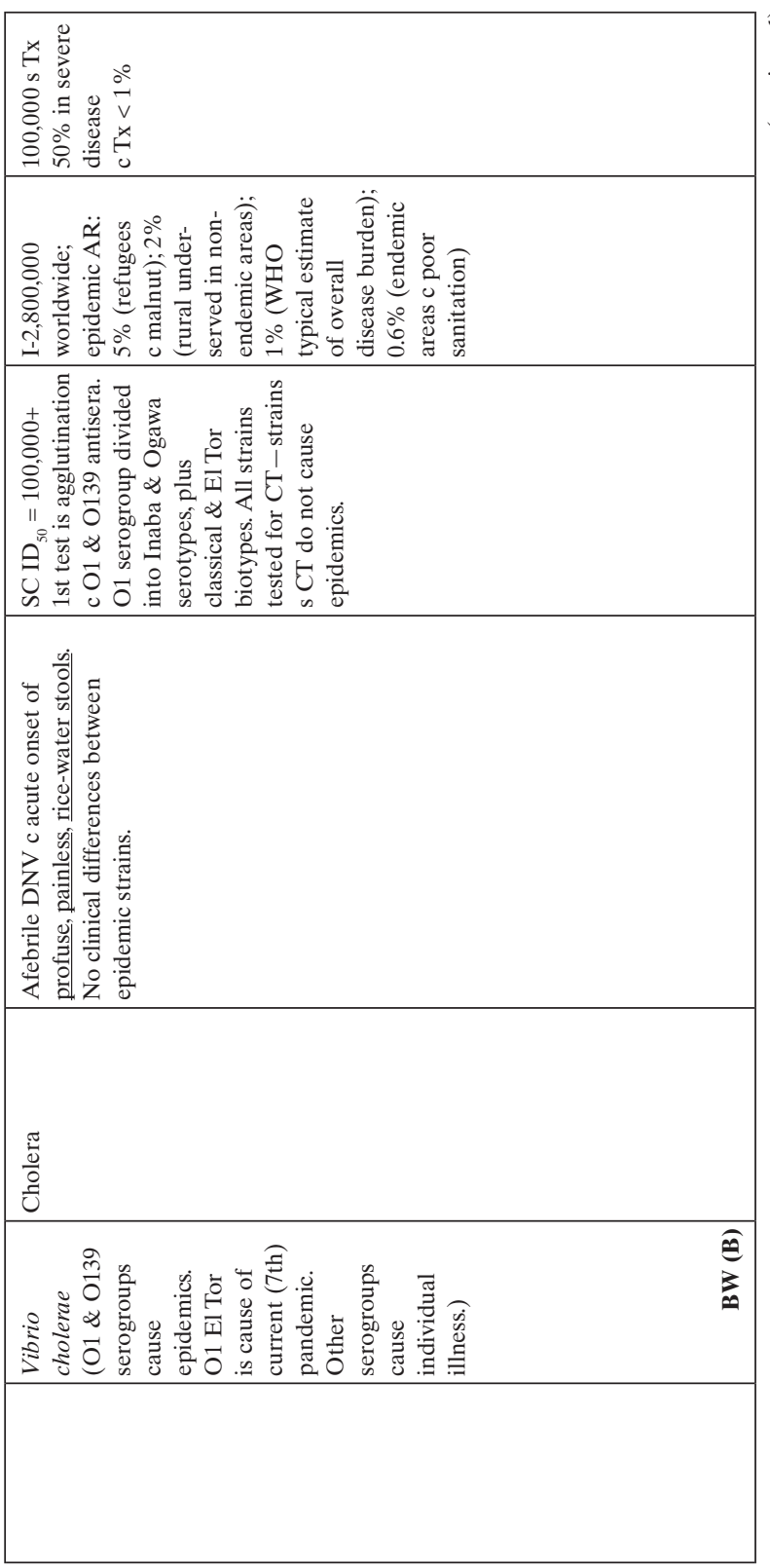







Document 7.4 223

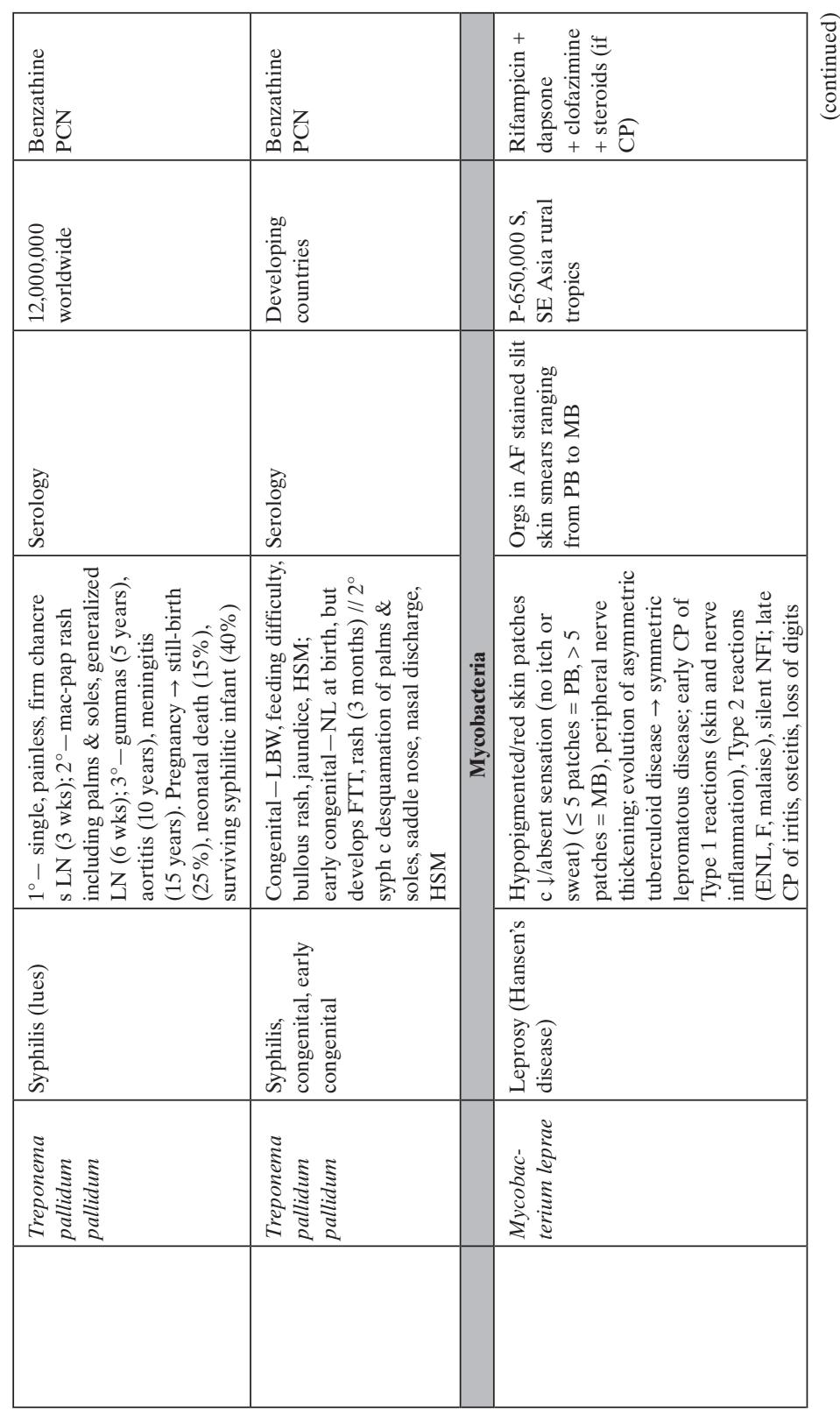




\begin{tabular}{|c|c|c|c|c|c|c|}
\hline 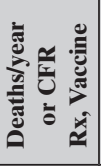 & & 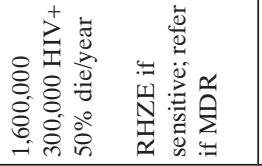 & 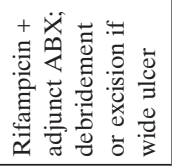 & & 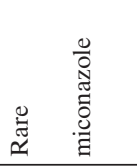 & 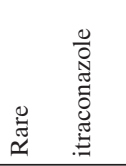 \\
\hline 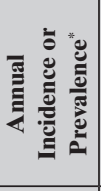 & & 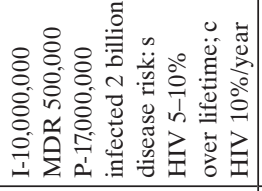 & 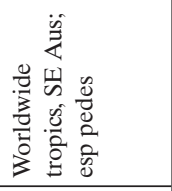 & & 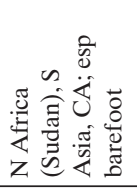 & 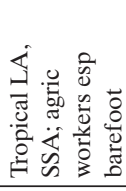 \\
\hline \multirow{3}{*}{ 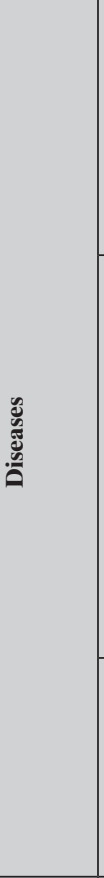 } & 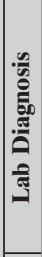 & 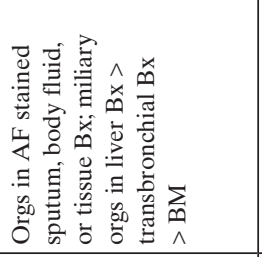 & 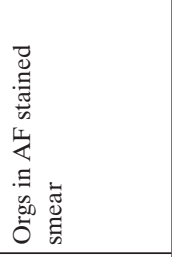 & \multirow[b]{2}{*}{ 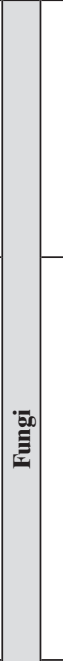 } & 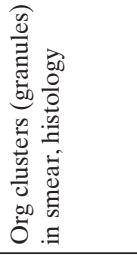 & 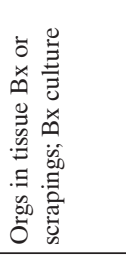 \\
\hline & 氖 & 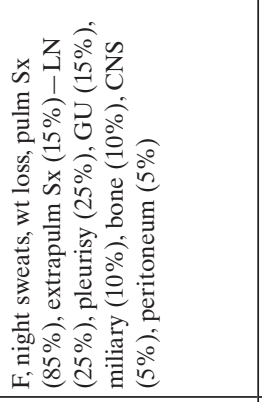 & 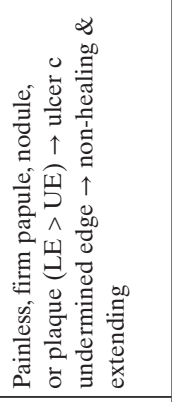 & & 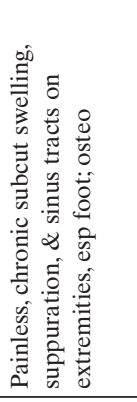 & 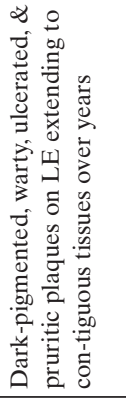 \\
\hline & 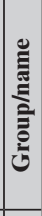 & 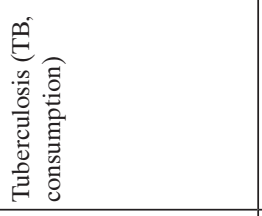 & 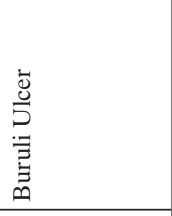 & 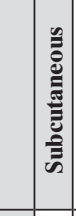 & 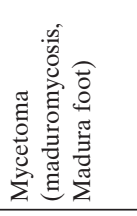 & 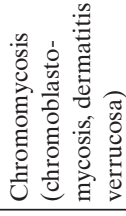 \\
\hline 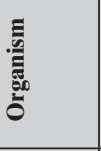 & & 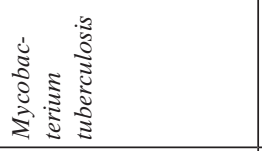 & 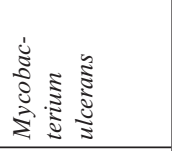 & & 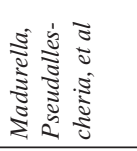 & 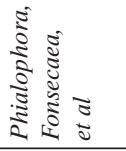 \\
\hline 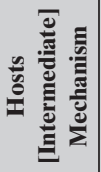 & & & & & & \\
\hline
\end{tabular}


Document 7.4 225

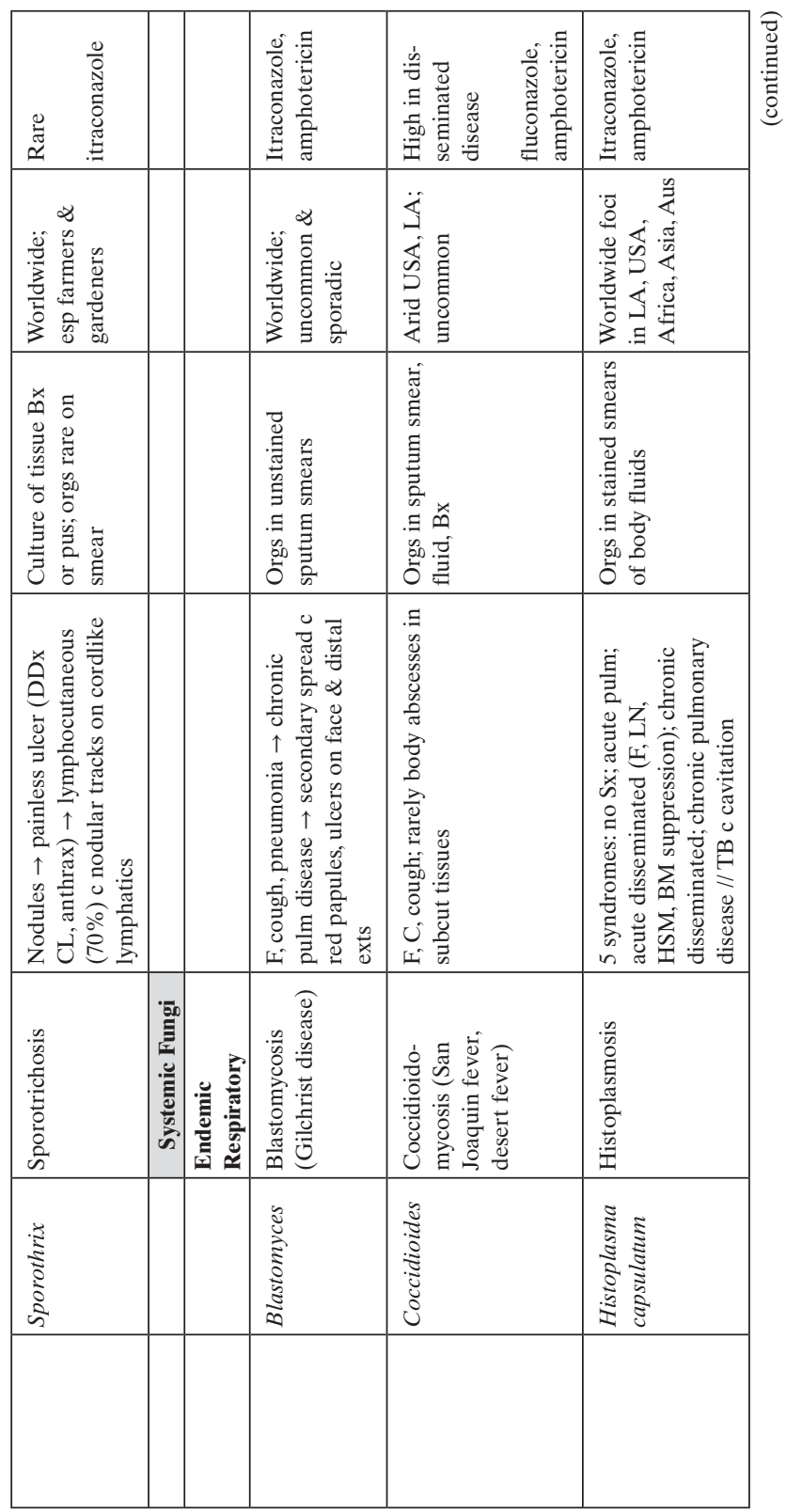




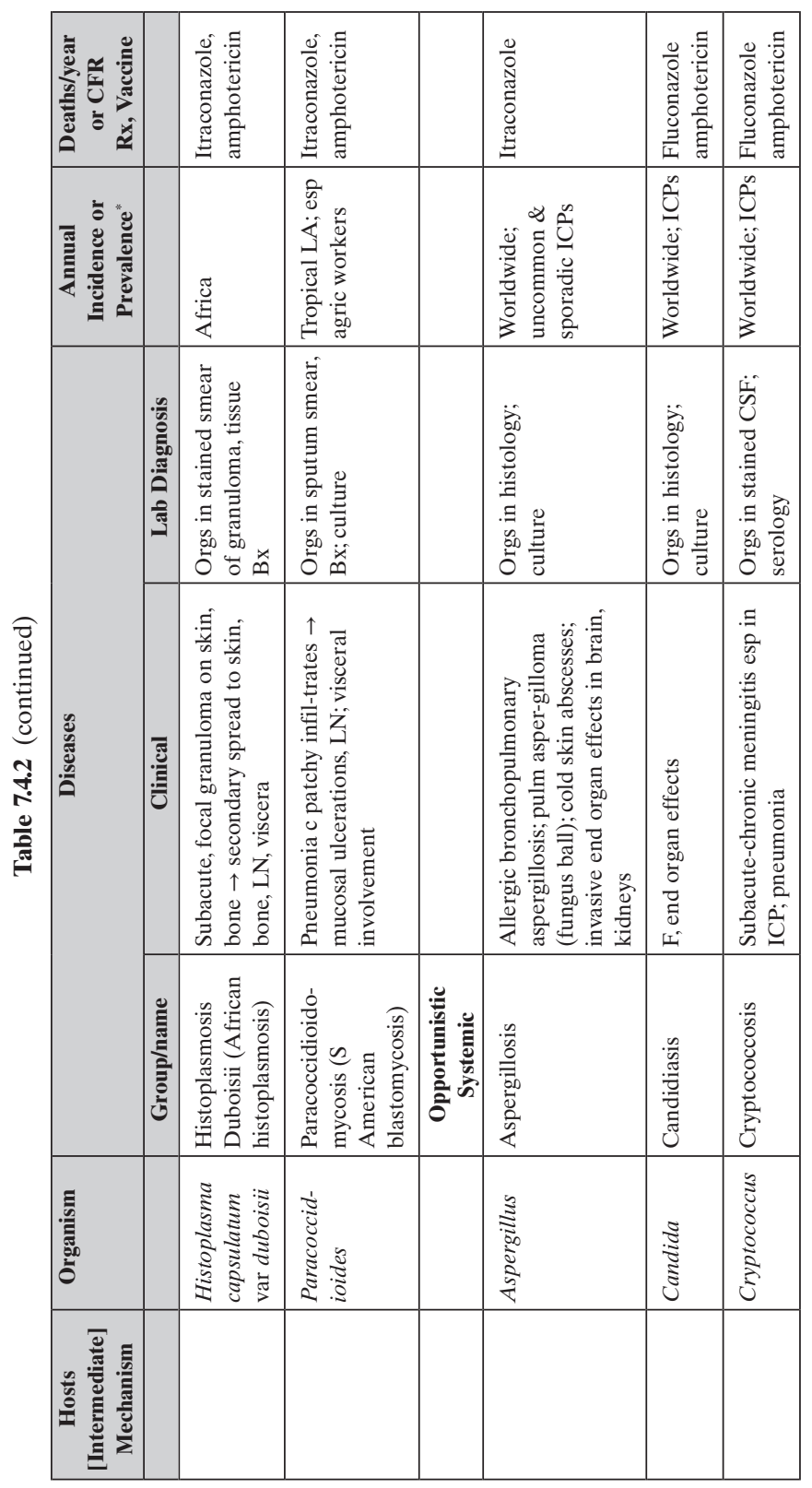


Document 7.4 227

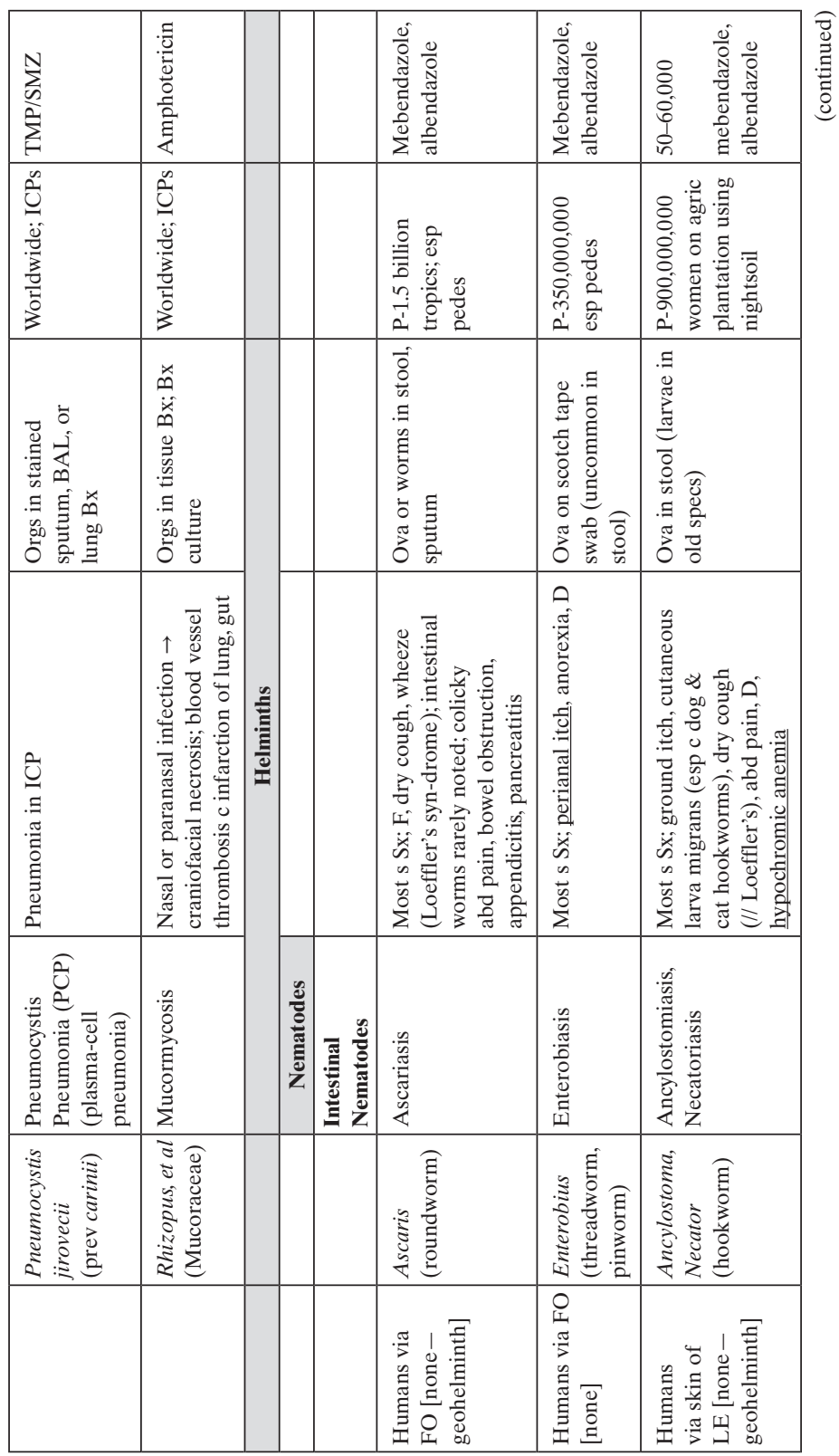




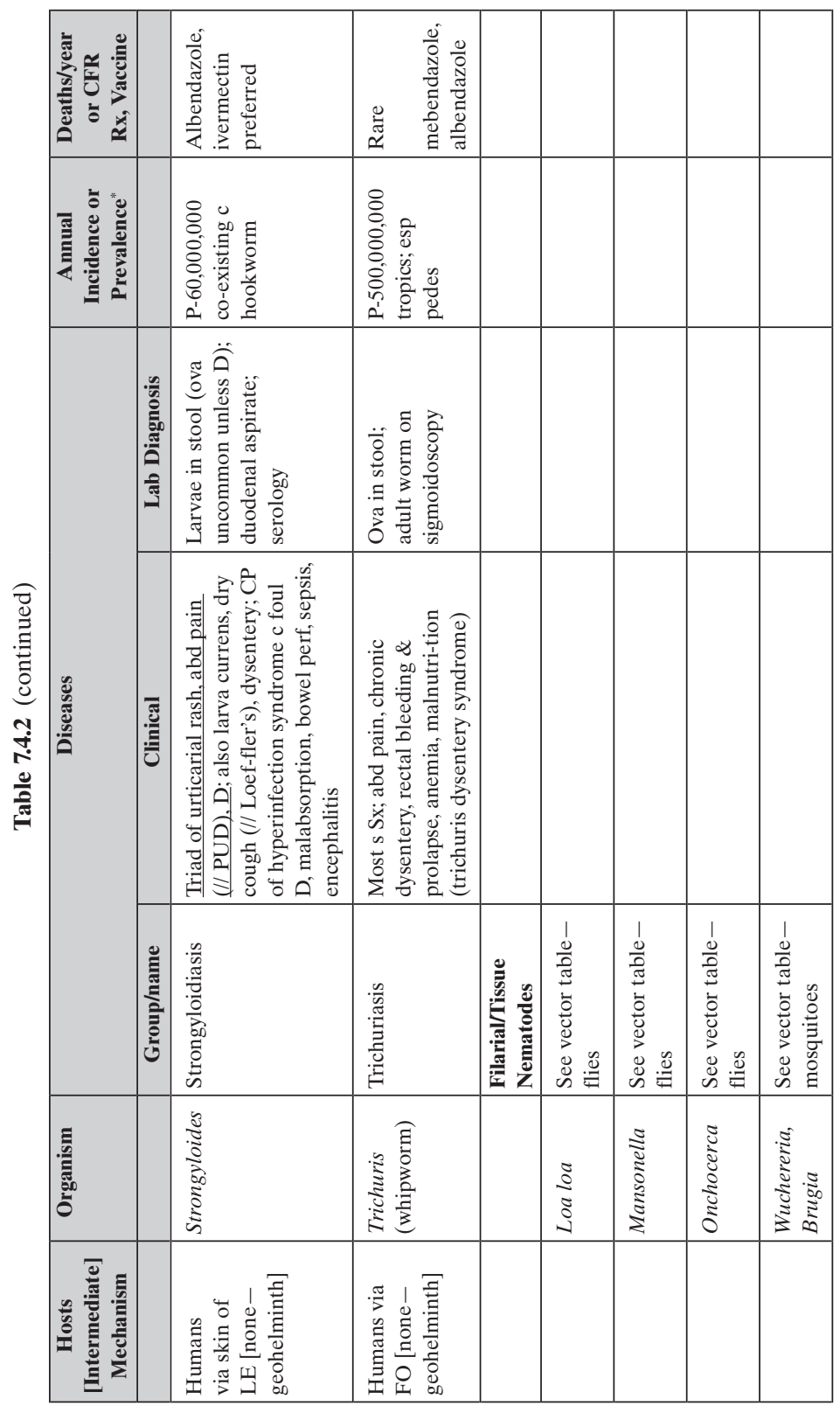


Document 7.4 229

\begin{tabular}{|c|c|c|c|c|c|c|}
\hline & 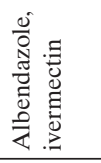 & 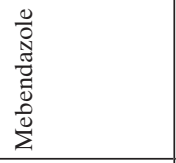 & 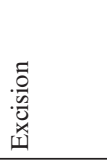 & 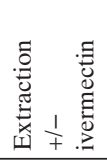 & 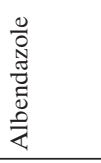 & 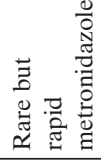 \\
\hline & & 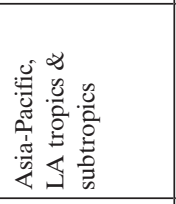 & 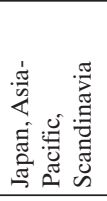 & 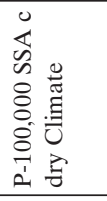 & 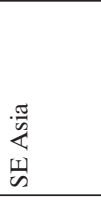 & 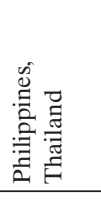 \\
\hline & $\begin{array}{l}0 \\
\tilde{0} \\
z\end{array}$ & 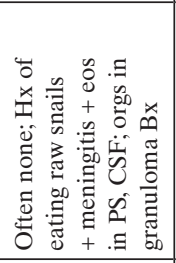 & 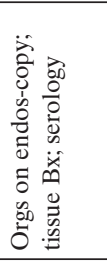 & $\begin{array}{l}\text { : } \\
\text { ż }\end{array}$ & 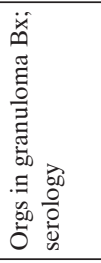 & 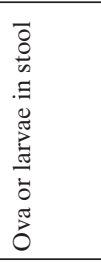 \\
\hline & 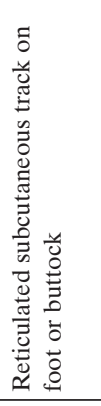 & 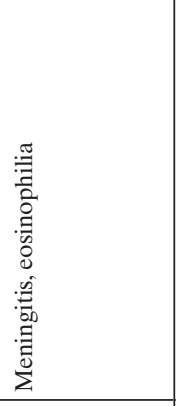 & 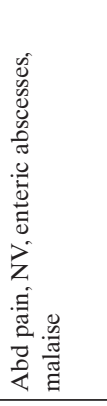 & 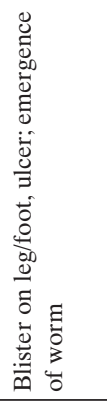 & 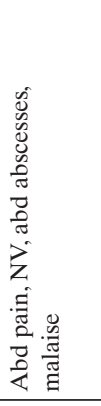 & 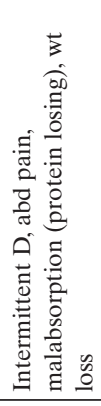 \\
\hline 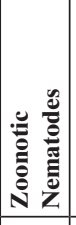 & 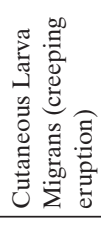 & 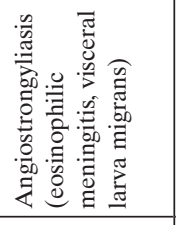 & 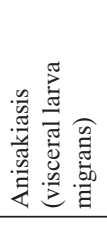 & 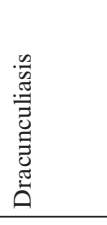 & 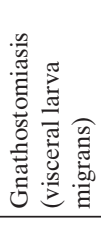 & 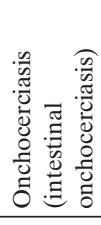 \\
\hline & 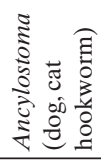 & 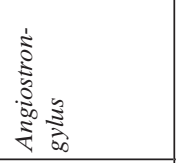 & 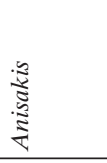 & 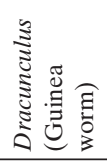 & 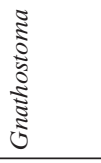 & 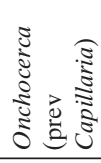 \\
\hline & 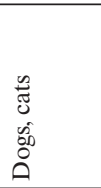 & 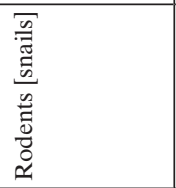 & 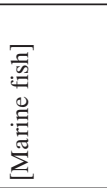 & 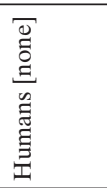 & 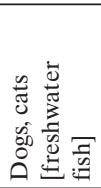 & 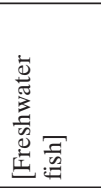 \\
\hline
\end{tabular}




\begin{tabular}{|c|c|c|c|c|c|c|c|}
\hline 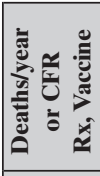 & & 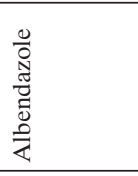 & 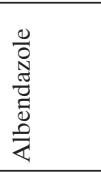 & & 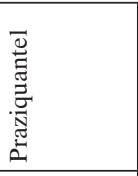 & 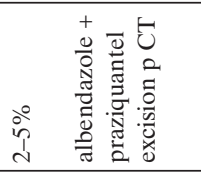 & 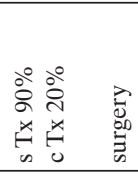 \\
\hline 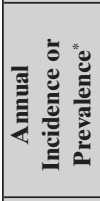 & & 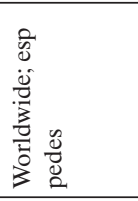 & $\begin{array}{l}8 \\
0 \\
0 \\
0 \\
0 \\
0 \\
1 \\
\end{array}$ & & 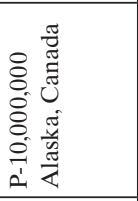 & 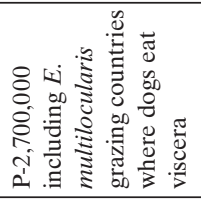 & 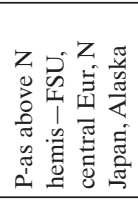 \\
\hline \multirow{3}{*}{ 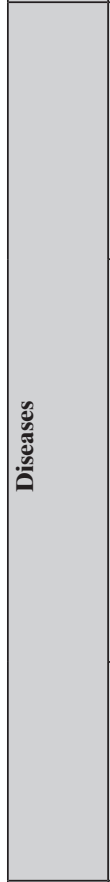 } & 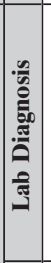 & 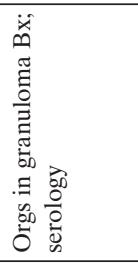 & 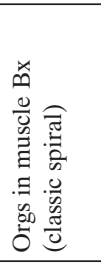 & & & 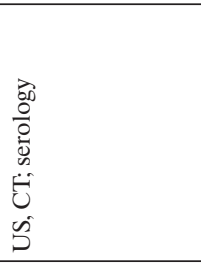 & 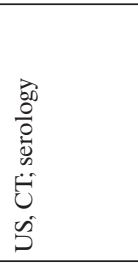 \\
\hline & "ृ. & 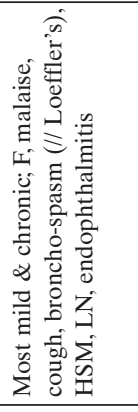 & 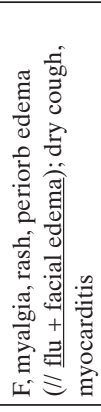 & & 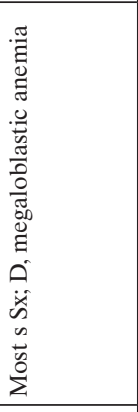 & 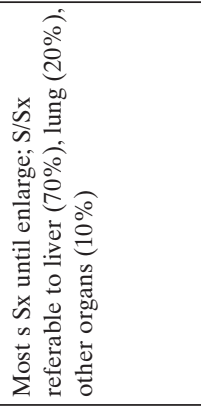 & 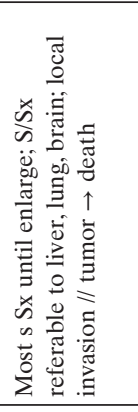 \\
\hline & | & 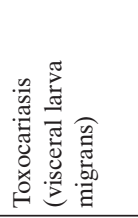 & 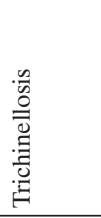 & Uू & 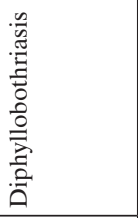 & 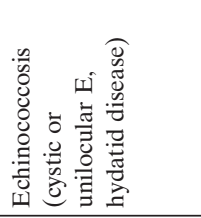 & 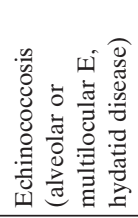 \\
\hline हू & & 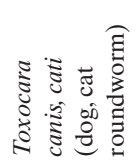 & 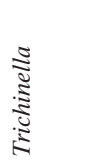 & & 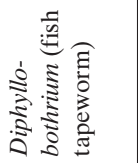 & 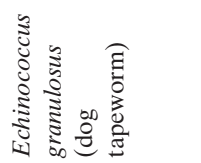 & 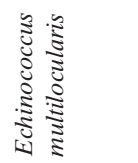 \\
\hline 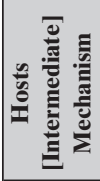 & & $\begin{array}{l}\frac{\omega}{\tilde{U}} \\
w_{0}^{0} \\
\stackrel{0}{0}\end{array}$ & 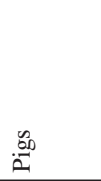 & & 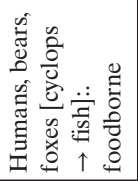 & 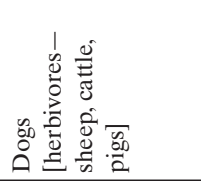 & 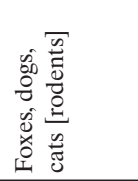 \\
\hline
\end{tabular}


Document $7.4 \quad 231$

\begin{tabular}{|c|c|c|c|c|c|}
\hline 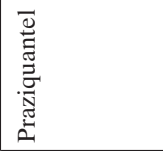 & 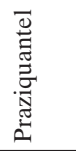 & 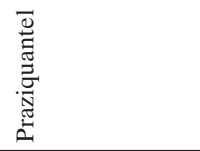 & & 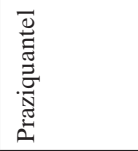 & 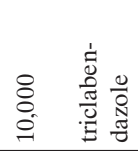 \\
\hline 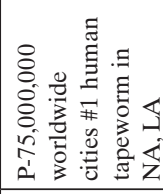 & 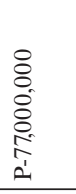 & $\begin{array}{l}8 \\
0 \\
0 \\
0 \\
0 \\
\stackrel{1}{1} \\
.1\end{array}$ & & 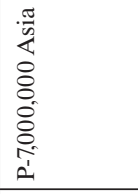 & 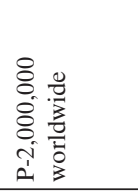 \\
\hline $\begin{array}{l}\overline{0} \\
0 \\
0 \\
. \Xi \\
\pi \\
0 \\
0\end{array}$ & 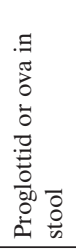 & 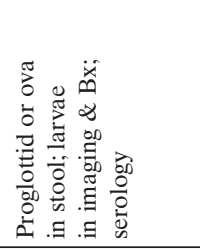 & & 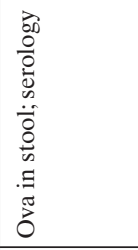 & 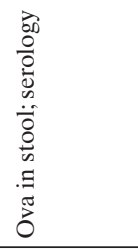 \\
\hline 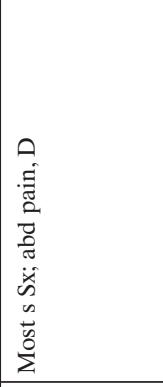 & 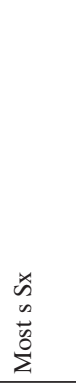 & 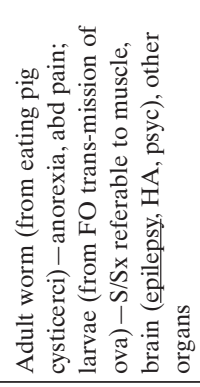 & & 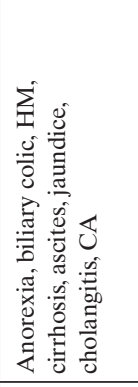 & 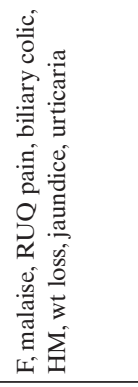 \\
\hline 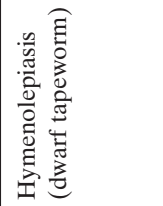 & 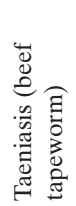 & 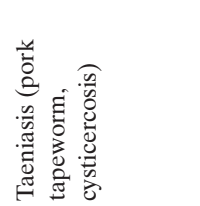 & 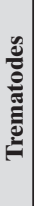 & 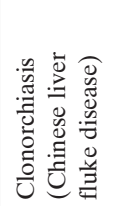 & 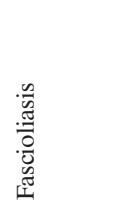 \\
\hline 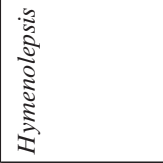 & 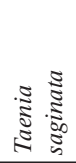 & 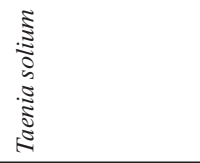 & & 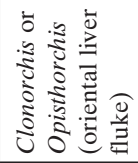 & 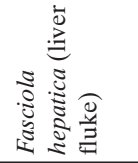 \\
\hline 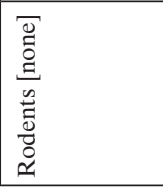 & 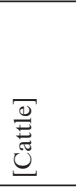 & $\begin{array}{l}\bar{w} \\
\stackrel{5}{0} \\
\ddot{E}\end{array}$ & & 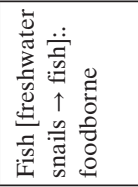 & 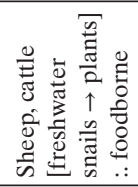 \\
\hline
\end{tabular}




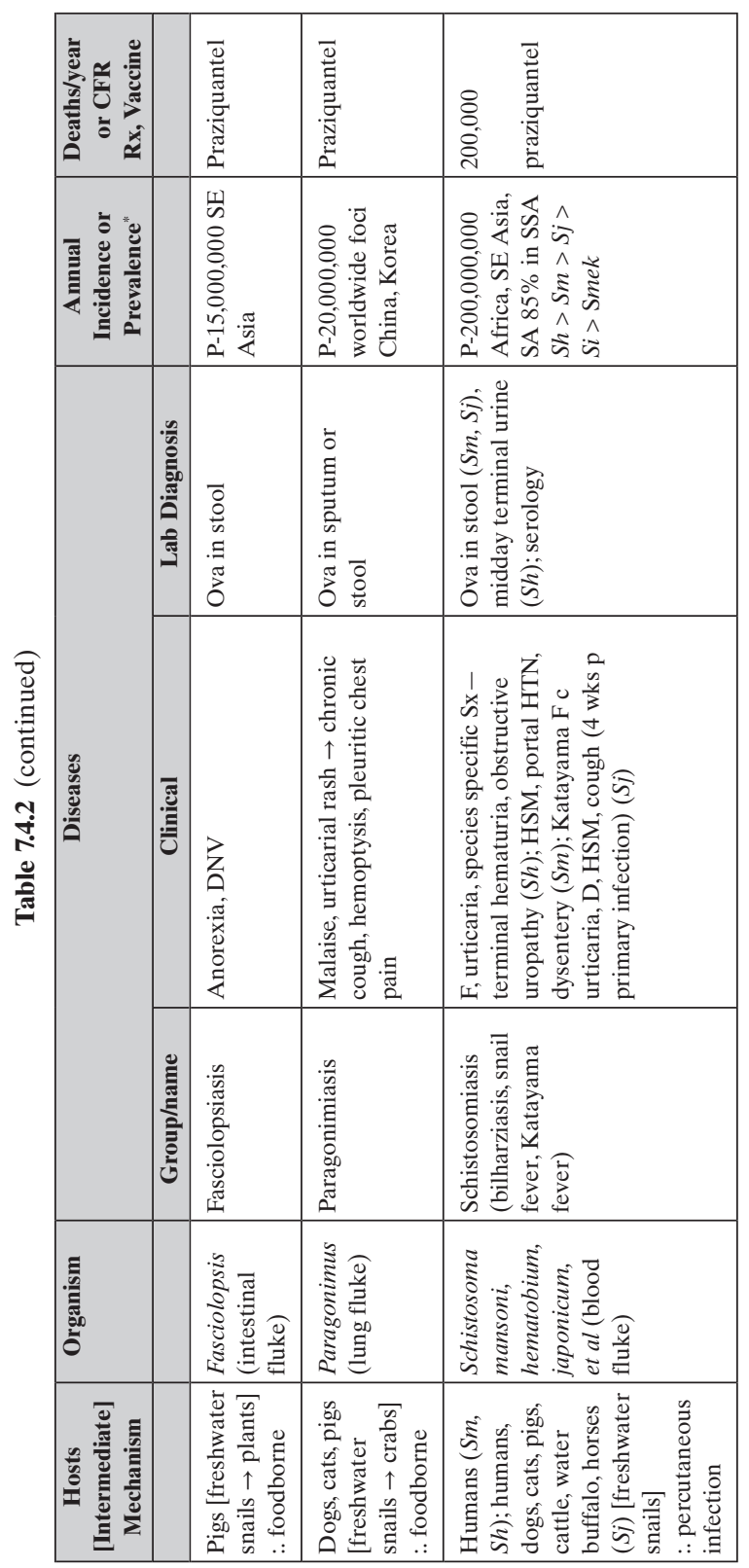


Document 7.4 233

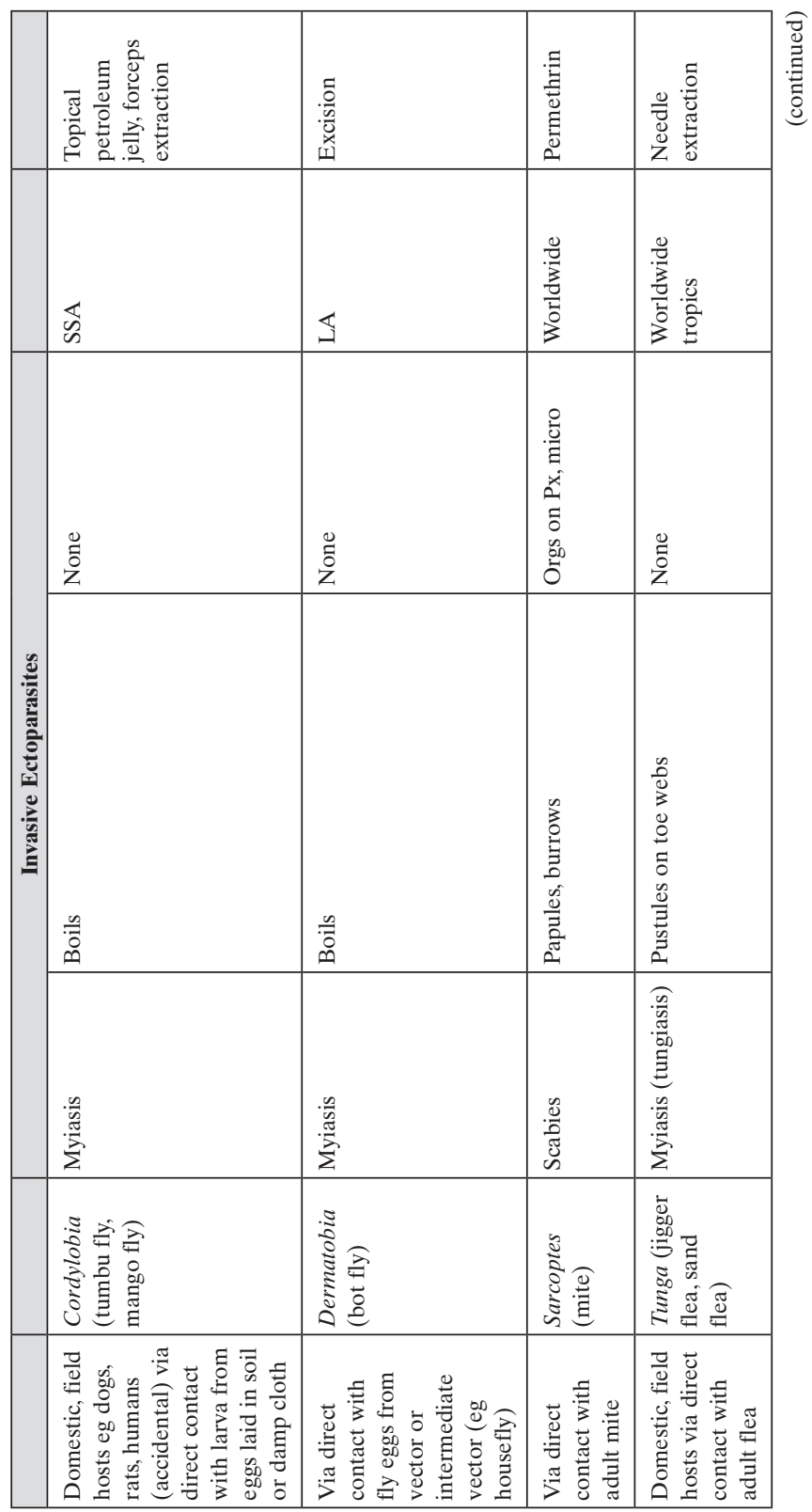


234 7. Technical Annexes

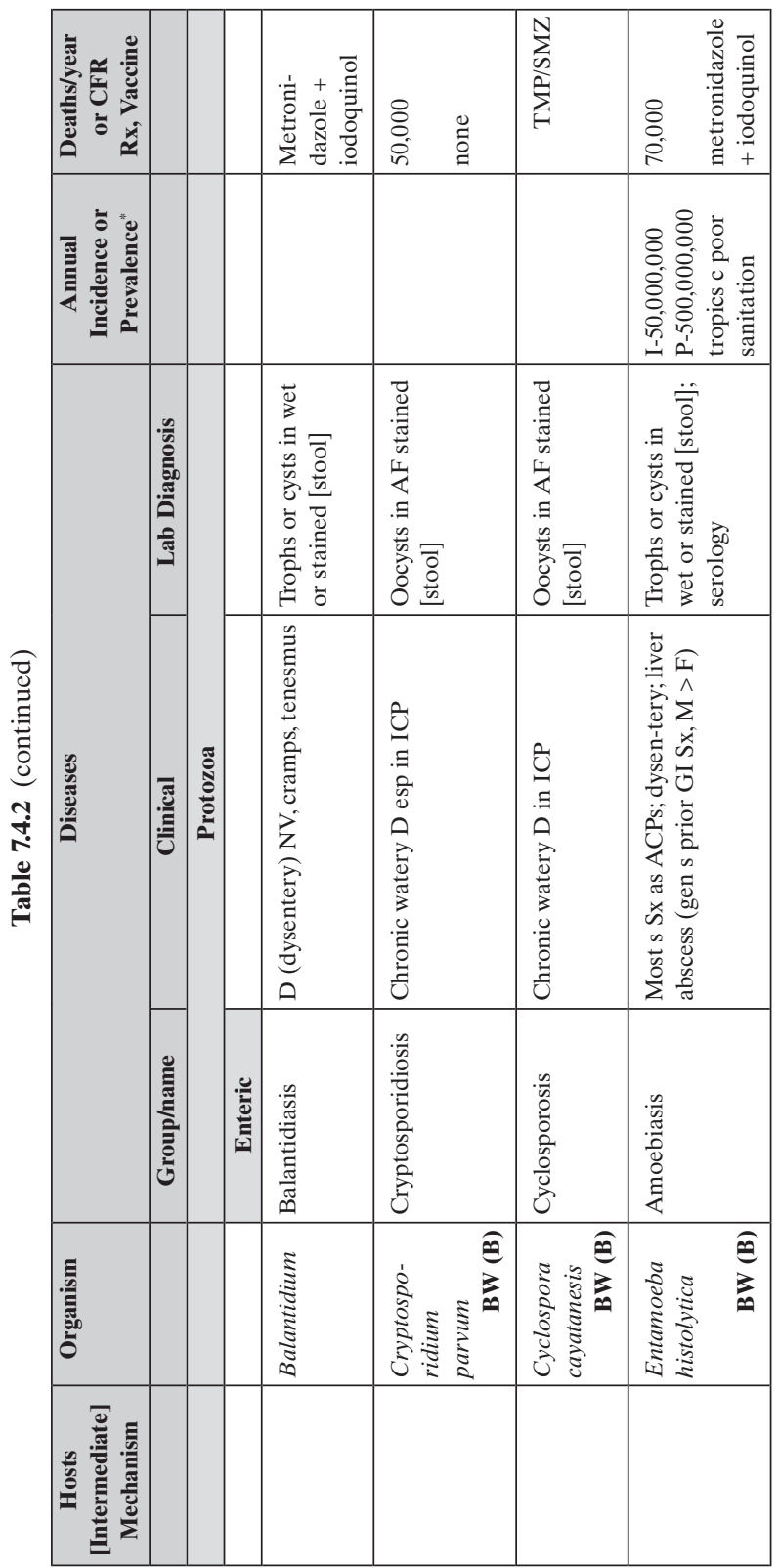


Document $7.4 \quad 235$

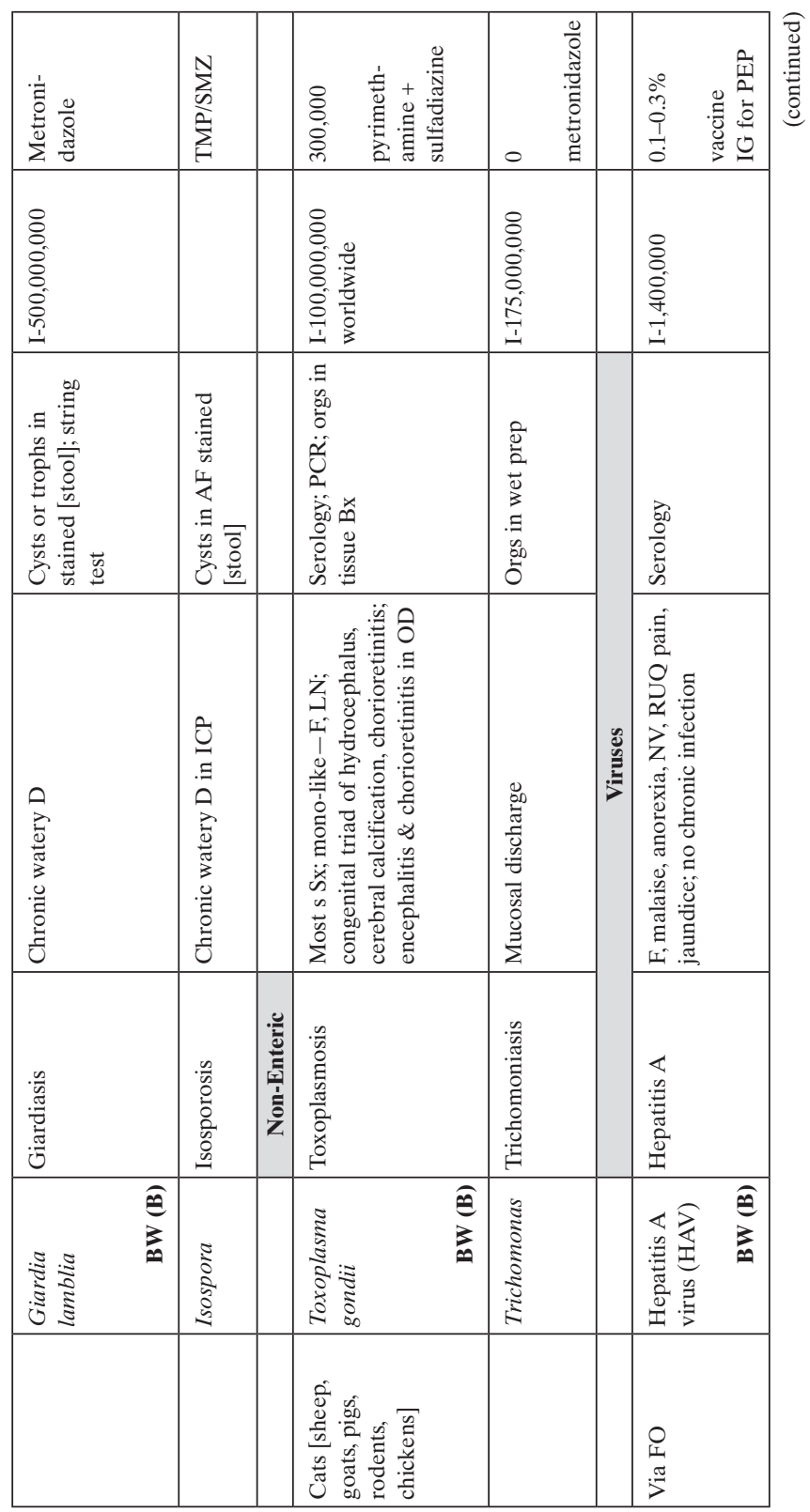







Document 7.4 237

\begin{tabular}{|c|c|c|c|}
\hline 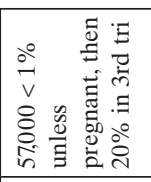 & 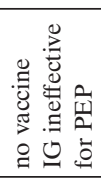 & 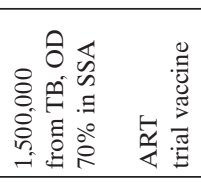 & 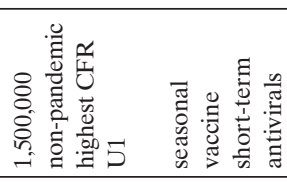 \\
\hline 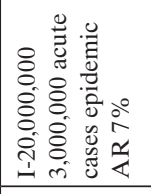 & & 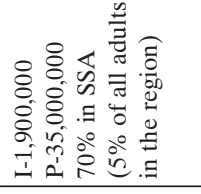 & 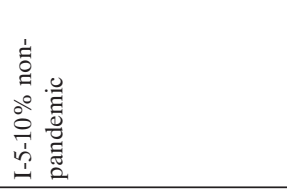 \\
\hline $\begin{array}{l}30 \\
\frac{0}{0} \\
\frac{0}{0} \\
0 \\
0\end{array}$ & & 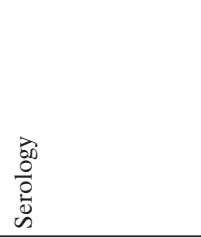 & 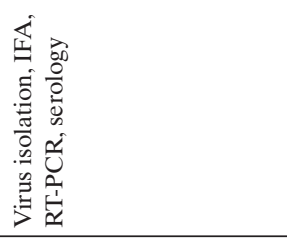 \\
\hline 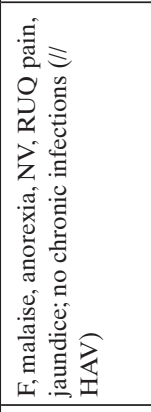 & & 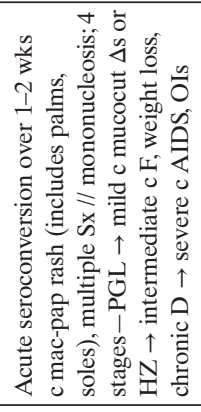 & 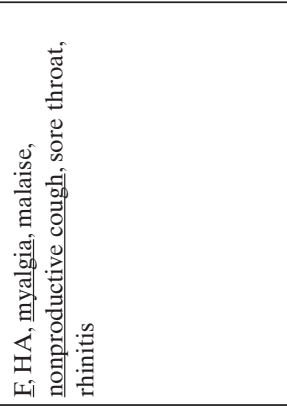 \\
\hline 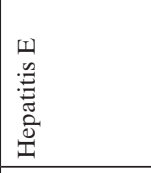 & & 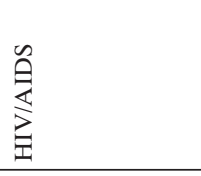 & 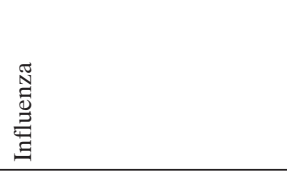 \\
\hline 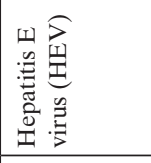 & & 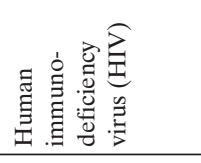 & 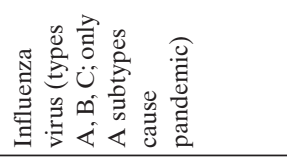 \\
\hline 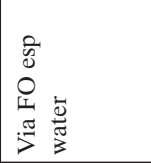 & & 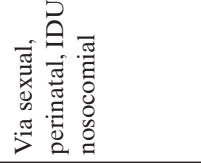 & 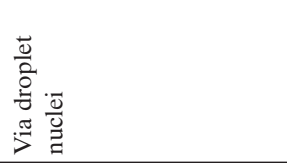 \\
\hline
\end{tabular}



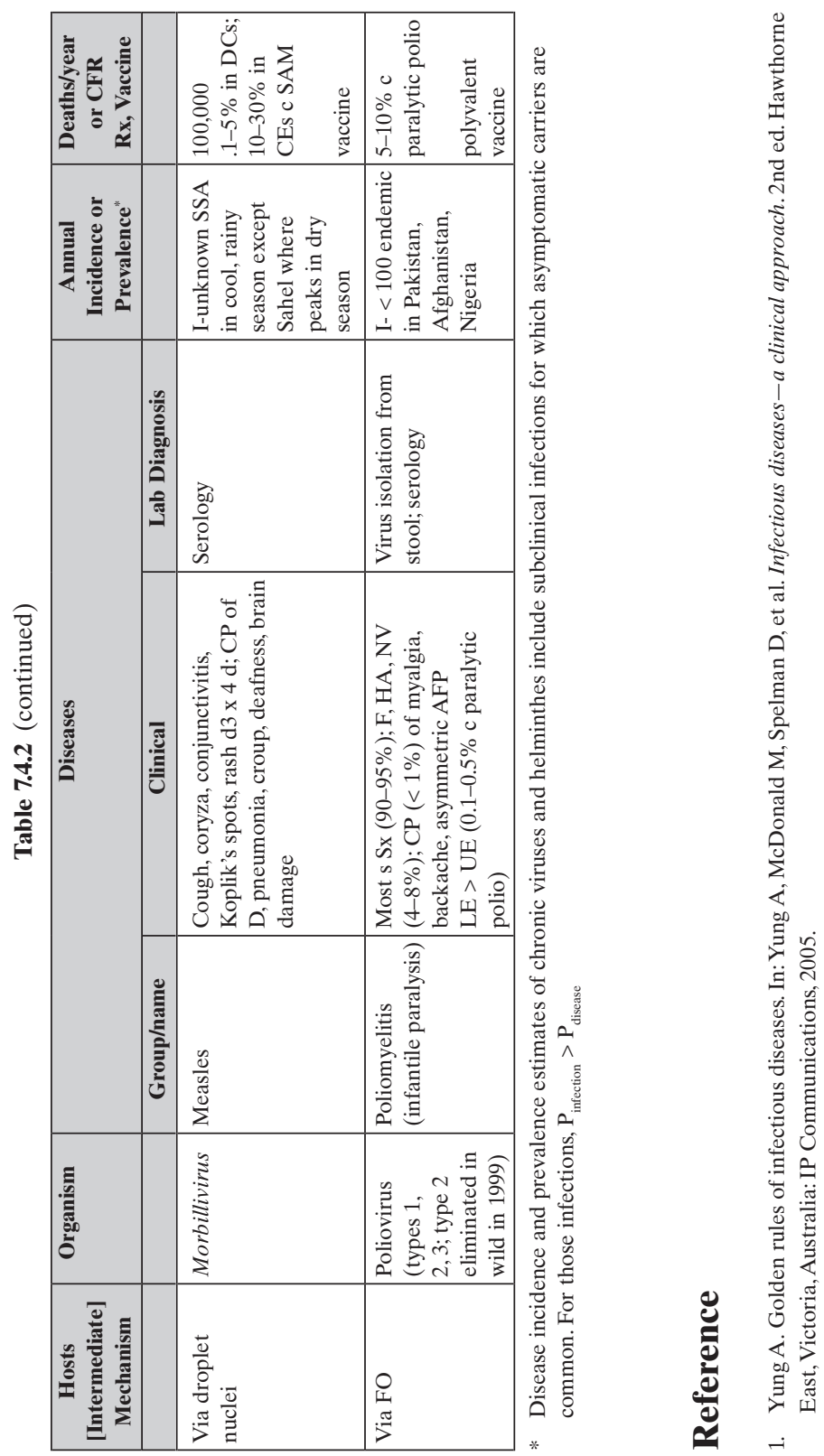


\section{Document 7.5 \\ EPIDEMIC PREPAREDNESS \\ AND RESPONSE}

\section{EPIDEMIC PREPAREDNESS}

\section{A. Prior to Seasonal Epidemic}

1. Establish a National Coordinating Committee (NCC).

2. Designate a lead agency and lead official in the NCC.

3. Establish a Local Coordinating Committee (LCC).

4. Designate a lead official in the LCC.

5. Anticipate roles for partner agencies (eg inter-agency and team coordination, disease surveillance, field epidemiological investigation, laboratory identification, case management guideline development, outbreak logistics, public information, and social mobilization).

6. Identify sources of funds.

7. Intensify disease surveillance.

8. Identify reference lab(s) for communicable diseases of epidemic potential.

9. Ensure mechanism for specimen transport.

\section{OUTBREAK RESPONSE}

\section{A. Initial Response to Suspected Outbreak}

1. Form an emergency team to investigate and manage the outbreak

a. identify key roles on the outbreak investigation team(s)

(1) epidemiology and surveillance

(2) case management

(3) water and sanitation

(4) laboratory services

(5) communication

b. staff those roles

(1) epidemiologist - to monitor proper data collection and surveillance procedures

(2) physician - to confirm clinical S/Sx and train health workers in case management

(3) water and sanitation expert - to develop a plan for reducing sources of contamination

(4) microbiologist - to take environmental/biological samples for laboratory confirmation, train health workers in proper sampling techniques, and confirm use of appropriate methods in the diagnostic laboratory

(5) behavior change communication (BCC) specialist - to assess the population's reaction to the outbreak, create, and disseminate appropriate health messages 


\section{B. Outbreak Investigation Protocol}

1. Establish access, contacts, logistics.

2. Verify outbreak.

3. Confirm diagnosis.

4. Develop case definition.

a. What are most patients complaining of?

b. Describe a typical patient.

c. Choose a case definition from the community descriptions confirmed by your own observations.

5. Count cases and determine demographic data.

a. How many people live in the outbreak area?

b. Who are the patients?

c. What is their background-age, sex?

d. Where are the patients coming from?

e. When did the patients arrive?

f. Why are the patients arriving?

g. Count the number of patients fitting the case definition.

h. Count the number of fresh graves or bodies at health facilities and inquire as to cause.

6. Orient the descriptive data-person, place, and time.

a. Tabulate data on affected patients.

b. Make a spot map.

(1) When and where was/were the first reported case(s) seen indicating an outbreak?

c. Plot an epidemic curve.

(1) What is the present \# of patients/day or week?

(2) What is the usual \# of patients/day or week?

(3) Is this an increase?

(4) What is the present \# of deaths/week or month?

(5) What is the usual \# of deaths/week or month?

(6) Is this an increase?

d. Calculate attack rates and case fatality ratios for total patients, U5, O5, and gender.

7. Develop hypothesis.

a. Postulate sources of disease and mechanism of spread.

b. Estimate the population at risk of contracting disease and of dying from it.

Consider especially:

(1) poor

(2) those with limited access to health services

(3) minorities

(4) malnourished

(5) pregnant and lactating

(6) infants not breast fed, children unvaccinated

(7) elderly 
8. Initiate control measures considering agent, host, and environment.

a. What action has the community taken?

b. Identify local response capacity.

(1) What number and type of staff are locally available?

(2) What drugs and supplies are locally available?

c. Determine immediate unmet needs.

(1) specimen collection and lab diagnosis

(2) logistics

(3) support for clinical care - staff, drugs, and supplies

(4) support for environmental health

d. Undertake further necessary actions.

(1) case management with secondary prevention

(2) patient isolation

(3) health education

(4) agent and reservoir identification

(5) environmental decontamination

(6) primary prevention

(7) public information

9. Inform authorities with investigation report.

10. Initiate ongoing disease surveillance.

\section{During Epidemic}

1. NCC should meet at least weekly.

2. LCC should meet daily at first, then reduce meeting frequency as circumstances warrant.

\section{Surveillance Systems Lessons Learned}

1. Start small, keep it simple

a. active surveillance at sentinel sites

b. simple standard operating procedures

(1) case investigation

(2) specimen collection

2. Invest in local people and systems

3. Focus the system on performance-based indicators

a. polio-non-polio acute flaccid paralysis detection rate of 1:100,000

b. gastroenteritis - stool specimen collection rate of $80 \%$

4. Link data to action

a. case detection $\rightarrow$ local response

b. case locations $\rightarrow$ need for (sub)national immunization days

c. lab info $\rightarrow$ recognition of imported vs indigenous cases

d. other process indicators $\rightarrow$ system improvement

5. Show success, then expand 


\section{Document 7.6 \\ COMMUNICABLE DISEASE CONTROL}

\section{Glossary}

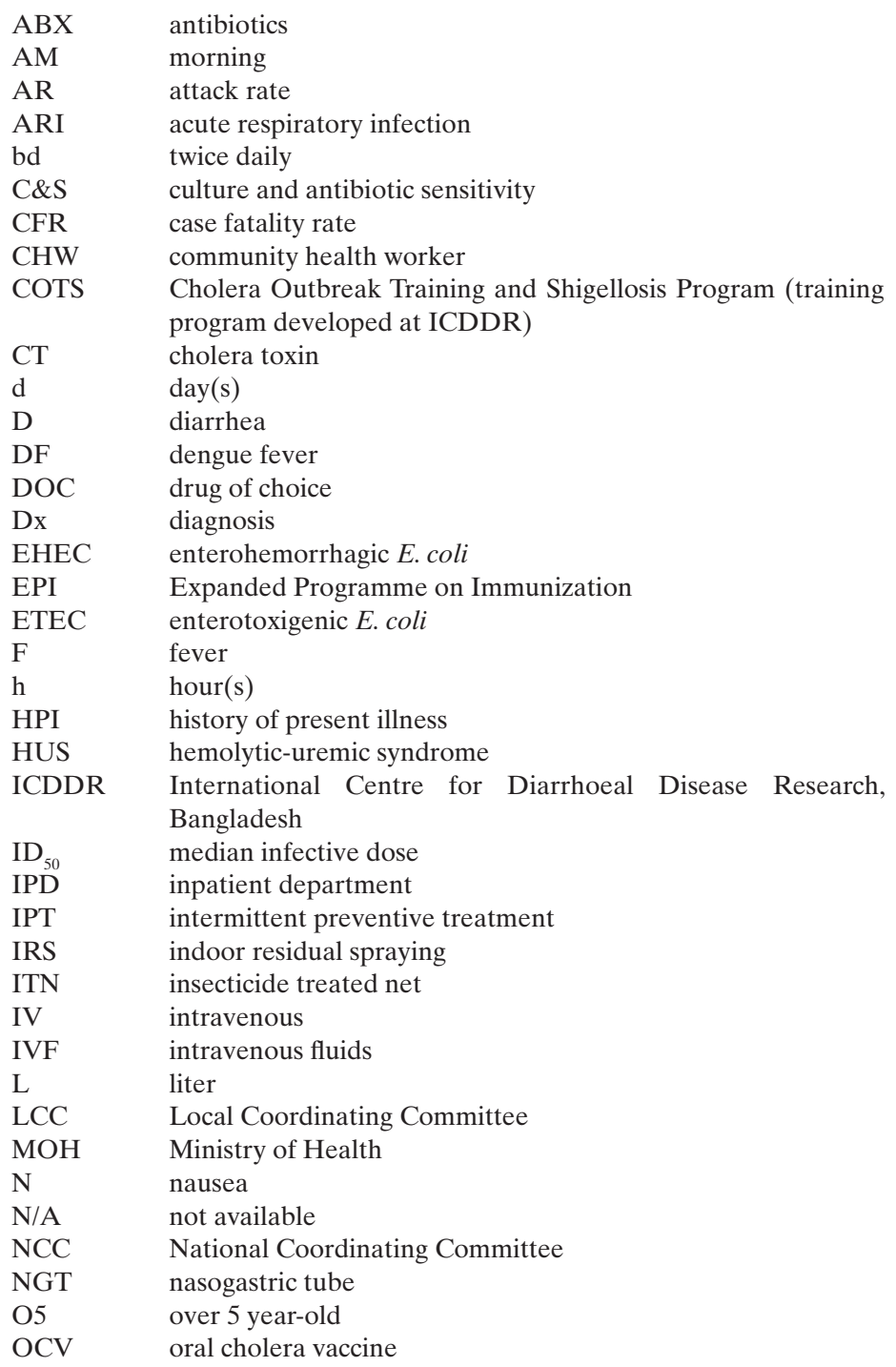




$\begin{array}{ll}\text { OPD } & \text { outpatient department } \\ \text { ORS } & \text { oral rehydration salts/solution } \\ \mathrm{p} & \text { person } \\ \mathrm{PO} & \text { by mouth } \\ \mathrm{PPE} & \text { personal protective equipment } \\ \mathrm{PRN} & \text { as needed } \\ \mathrm{Pt}(\mathrm{s}) & \text { patient(s) } \\ \mathrm{P}_{\mathrm{T}} & \text { population total } \\ \mathrm{q} & \text { every } \\ \mathrm{qd} & \text { daily } \\ \mathrm{rBS} & \text { recombinant beta subunit (of cholera toxin) } \\ \mathrm{RDT} & \text { rapid diagnostic test } \\ \mathrm{SD} 1 & \text { S. dysenteriae type 1 } \\ \mathrm{SMC} & \text { seasonal malaria chemoprophylaxis } \\ \mathrm{SSU} & \text { short stay unit } \\ \mathrm{S} / \mathrm{Sx} & \text { signs and symptoms } \\ \mathrm{STEC} & \text { Shiga toxin producing } \text { E.coli } \\ \mathrm{Sx} & \text { symptoms } \\ \mathrm{T} & \text { temperature } \\ \mathrm{U} 2 & \text { under 2 year-old } \\ \mathrm{U} 5 & \text { under 5 year-old } \\ \mathrm{V} & \text { vomiting } \\ \text { WHO } & \text { World Health Organization } \\ \text { YF } & \text { yellow fever } \\ \text { yr } & \text { year(s) } \\ \text { Zn } & \text { zinc } \\ \end{array}$

\section{Contents}

This Annex profiles selected communicable diseases of epidemic potential whose incidence, management complexity, or mortality obliges particular attention.

- Diarrhea

- Influenza

- Malaria

- Measles

- Meningitis

- Viral Hemorrhagic Fever

The profiles are structured to quickly orient field staff to key issues in:

- Pathogens

- Epidemiology

- Preventive Medicine

- Epidemiological Surveillance

- Clinical Medicine

- Epidemic management 


\section{DIARRHEA}

\section{Pathogens}

\section{Cholera}

$V$. cholerae has $>200$ serogroups. Serogroups are classified by biotype, and for serogroup O1, by serotype and biotype. Humans are the only known vertebrate host for cholera, and only serogroups O1 and O139 cause epidemic disease. There is no clinical difference between them. Other serogroups may cause disease in individuals, but not epidemics. When a suspected cholera serotype (strain) is isolated in the lab, one of the first tests performed is bacterial agglutination with $\mathrm{O} 1$ and $\mathrm{O} 139$ antisera. Strains are thereby identified as $V$. cholerae O1, O139, or non-O1/ non-O139.

- If (+) agglutination to O1 antisera, then the strain is further tested for agglutination to antiserum of Ogawa and Inaba serotypes.

- If (+) agglutination to O139 antisera, then the strain is not further subdivided (except as producer or non-producer of CT as noted below).

- If (-) agglutination to O1 and O139 antisera, then the strain is known as non-O1, non-O139 $\mathrm{V}$. cholerae.

A strain is further identified as a producer or non-producer of cholera toxin (CT). CT production is a major determinant of disease development. Strains lacking CT do not produce epidemics even if from the O1 or O139 serogroup.

- Serogroup O1 exists as 2 main biotypes-classical and El Tor-though hybrids also exist. Each biotype occurs as two serotypes-Ogawa and Inaba. Classic biotype caused the 5th and 6th pandemics but little epidemic disease since the 1970s though it still causes cases in India. EI Tor biotype caused the 7 th (current) pandemic and almost all recent outbreaks. El Tor was first isolated in 1905 in El Tor, Egypt after importation by Indonesian pilgrims travelling to Mecca. It survives longer in the environment and produces CT similar to the classical biotype. Presumably because of CT pathogenicity, the \% of cholera patients with severe disease has doubled over the past $10 \mathrm{yrs}$. These patients tend to require IV fluid therapy.

- Serogroup O139 may have evolved from strains of O1 El Tor as they share many properties though not agglutination. In spring of 2002 in Dhaka, O139 cases exceeded O1 El Tor cases for the first time, and it was postulated that O139 may become the cause of an 8th pandemic. However, since then, $\mathrm{O} 1$ has again become dominant.

Infective dose depends on individual susceptibility. Relevant host factors include immunity produced by prior infection with serogroup $\mathrm{O} 1$ as well as stomach acidity. ID $_{50}$ may be 100,000 orgs, so personal hygiene plays a lesser role than in shigellosis where the $\mathrm{ID}_{50}$ is much lower. 
Shigella

Shigella has 4 species.

- S. dysenteriae type 1 (SD1 or Shiga bacillus) causes the severest disease of all Shigella sp because of its neurotoxin (Shiga toxin), longer duration of illness, higher ABX resistance, higher CFR thru invasive complications, and great epidemic potential.

- S. flexneri is the most common, and is generally endemic, in developing countries

- S. sonnei is the most common in industrial countries

- S. boydii and S. sonnei give mild disease.

Table 7.6.1

Shigella Species

\begin{tabular}{|l|l|l|l|}
\hline \multicolumn{1}{|c|}{ Species } & \multicolumn{1}{|c|}{ Serogroup } & \multicolumn{1}{c|}{ Serotypes } & \multicolumn{1}{c|}{ Notes } \\
\hline S. dysenteriae & A & $1-15$ & $\begin{array}{l}\text { SD1 gives most } \\
\text { severe disease with } \\
\text { complications of HUS }\end{array}$ \\
\hline S. flexneri & B & $\begin{array}{l}1-6(15 \\
\text { subtypes })\end{array}$ & $\begin{array}{l}\text { Greatest burden of } \\
\text { disease and main cause } \\
\text { of endemic shigellosis }\end{array}$ \\
\hline S. boydii & C & $1-18$ & Mild disease \\
\hline S. sonnei & D & 1 & Mild disease \\
\hline
\end{tabular}

$\mathrm{ID}_{50}$ may be 10 orgs, so personal hygiene plays a greater role than in cholera.

\section{E coli}

Some kinds of E. coli produce a Shiga toxin. Shiga toxin genes reside in a bacteriophage genome integrated into the bacterial chromosome. Some $\mathrm{ABX}$, eg fluoroquinolones, induce expression of phage genes. The bacteria that make these toxins are variously called "Shiga toxin-producing $E$. coli" (STEC), "enterohemorrhagic E. coli" (EHEC), or "verocytotoxic E. coli" (VTEC). All terms refer to the same group of bacteria.

- E. coli $\mathrm{O} 157: \mathrm{H} 7$ (often called "E. coli O157" or "O157") is the most commonly identified STEC in North America, and it causes most E. coli outbreaks. Approximately 5-10\% of EHEC infections result in HUS.

- Non-O157 STEC serogroups also cause disease. In the USA, serogroups $\mathrm{O} 26, \mathrm{O} 111$, and $\mathrm{O} 103$ are the most commonly identified E. coli pathogens overall. 


\section{Epidemiology}

Diarrhea epidemiology is seasonally dependent. Environmental temperature directly influences biologic activity $-\Delta 5{ }^{\circ} \mathrm{C}$ is proportional to $3 \times$ risk of disease

- temperate climates: bacterial diarrhea in warmer, humid season; rotavirus diarrhea in cooler, dry season

- tropical climates: bacterial diarrhea in rainy season; rotavirus diarrhea year round with increased incidence in cooler season

- most common pathogens for watery diarrhea-rotavirus, ETEC, V. cholerae; most important pathogen for epidemic watery diarrhea $-V$. cholerae

- most common pathogens for dysentery - shigella species, salmonella species, Campylobacter jejuni, Clostridium difficuile, EIEC, EHEC, E. coli O157:H7, Entamoeba histolytica, Yersinia enterocolitica; most important pathogens for epidemic dysentery - S. dysenteriae serotype 1 (developing countries), E. coli O157:H7 (developed countries)

Bangladesh has 2 seasonal cholera peaks: pre-monsoon with hot, humid weather (esp weeks 15-20 in Apr-May) creating increased biological activity; post-monsoon (esp weeks 30-40 in Aug-Sep) with contamination of water sources. Pre-monsoon epidemics are generally worse than postmonsoon ones. Dysentery has low level year-round incidence, but epidemics occur roughly each decade. Epidemic strains display new, additive antibiotic resistance which probably triggers the epidemic. Once resistant strains have become endemic, antibiotic susceptibility rarely reappears. SD1 acquires resistance quickly. $S f$ acquires it more slowly, and that resistance may wane with decreasing ABX pressure.

At ICDDR, annual proportional incidence approximates the following:

Table 7.6.2

Proportionial Incidence of Diarrheal Pathogens in Bangladesh

\begin{tabular}{|l|l|l|l|}
\hline \multicolumn{2}{|c|}{ Total } & \multicolumn{2}{c|}{ Pedes } \\
\hline Rotavirus & $30 \%$ & Rotavirus & $45 \%$ \\
\hline Cholera (O1 > > O139) & $20 \%$ & E. coli & $45 \%$ \\
\hline ETEC & $15 \%$ & Camplyobacter & $10 \%$ \\
\hline $\begin{array}{l}\text { Shigella (flexneri > > boydii, } \\
\text { sonnei, SD1) }\end{array}$ & $5 \%$ & & \\
\hline Other & $30 \%$ & & \\
\hline
\end{tabular}

- E. coli overall $=35 \%$ of cases, but $\mathrm{ETEC}=15 \%$.

- E. coli tends to dominate before monsoon season and flooding

- Cholera tends to dominate after monsoon season and flooding

- Overall, 60-70\% of diarrhea cases may be vaccine-preventable.

- $30 \%$ of Pts have no pathogen identified. 


\section{Preventive Medicine}

Clean water and waste management especially for cholera.

Personal hygiene (hand washing with soap and clean towels) especially for shigella.

Water safe drinking water (boiled, chlorinated)

NB Sphere standards are not enough - you need increased quantities of chlorinated water at household level.

San clean latrines for safe disposal of excreta

hand washing with soap

Food safe food (cooked, stored)

breast feeding

Fomites safe disposal of dead bodies with disinfection of clothing

NB After outbreak of a fecal-oral pathogen, food hygiene and funereal practices may influence human-to-human transmission more than water quality.

Health education to affected population

Wash hands with soap:

after using toilets/latrines.

after disposing of children's feces.

before preparing food.

before eating.

before feeding children.

Boil or disinfect water with chlorine solution. (Bottle it, boil it, ferment it, or forget it.)

Eat only freshly cooked food. (Peel it, cook it, or leave it.)

Do not defecate near water sources.

Use latrines and keep them clean.

Consider vaccination for areas and populations most at risk. Pre-emptive vaccination for new arrivals (esp nutritionally impaired IDPs) moving into an endemic area is increasingly accepted. Reactive vaccination where cholera has broken out is more controversial-particularly if vaccine stocks are not locally available and remain limited worldwide. There are 2 types of oral cholera vaccines licensed internationally: one with dead $V$. cholerae, and one with live V. cholerae. (Parenteral cholera vaccine is not recommended because of low protective efficacy and frequent severe adverse reactions.)

\section{killed whole cell $V$. cholerae 01 vaccine}

- Dukoral-mixture of the classical biotype (both Inaba and Ogawa serotypes) and the El Tor biotype (only Inaba serotype) + purified recombinant B-subunit of CT (rBS)

Dose: $3 \mathrm{cc}$ vaccine given in $150 \mathrm{cc}$ of buffer solution; 2 doses, $1-6 \mathrm{wks}$ apart; cold chain required. The regime is identical for all patients, and thus can't be given to pedes $<2 \mathrm{yr}$ because of volume loading. 
Dukoral has been the main vaccine considered for use in high-risk populations.

- mORC-VAX and Shanchol-similar to Dukoral except they do not contain the rBS, hence do not require a buffer, and are $1 / 3$ the cost to produce. mORC-VAX, produced in Vietnam, is derived from a vaccine administered to millions of people since 1997, but is not WHO pre-qualified, and is not expected to have international distribution.

- Shanchol, produced in India, has international distribution (eg used in the Haiti cholera vaccination campaign of 2012), and is now the agent of choice for WHO. It confers immunity $10 \mathrm{~d} \mathrm{p} 2 \mathrm{nd}$ dose, effectiveness $>85 \%$ at $6 \mathrm{mo}$, and protection $>50 \%$ at $5 \mathrm{yr}$. Also confers short-term protection vs ETEC.

Dose: $1.5 \mathrm{cc}$ vaccine followed by water ingestion but no fasting needed; 2 doses, 2 wks apart; cold chain required except for day of use.

\section{live-attenuated genetically modified $V$. cholerae 01 vaccine}

- Orochol-bivalent formulation as in Dukoral without rBS of CT.

Dose: single dose. No longer manufactured.

WHO recommendations: "Vaccination should not disrupt the provision of other high-priority health interventions to control or prevent cholera outbreaks. Vaccines provide a short-term effect that can be implemented to bring about an immediate response while the longer term interventions of improving water and sanitation, which involve large investments, are put into place." [1]

ICDDR recommendations: "Because of limitations in terms of transport, formulation, and cost of the current Dukoral vaccine, the COTS program does NOT require the utilization of the vaccine during an outbreak; it is NOT necessary to vaccinate to overcome an outbreak. However, if Dukoral is readily available and staff are properly trained in its use according to the guidelines that come with the vaccine, the COTS program PERMITS Dukoral's use (ideally before an outbreak) in the following high-risk populations: refugee populations in which cholera is present, health care workers managing cholera cases, and communities in which the incidence rate is greater than 1 in 1000 annually." [2]

\section{Epidemiological Surveillance (specific to cholera)}

\section{Cholera}

Epidemiological assumptions (WHO, COTS):

Estimated attack rates: $\quad 10-20 \%$ extremely vulnerable hosts and poor environmental health (WHO)

$5 \%$ (refugee camps with malnutrition) (COTS)

$2 \%$ (rural communities of $<5000 \mathrm{p}$ ) (COTS) 
$1 \%$ (severe epidemic-good estimate of ultimate disease burden) (WHO)

$0.6 \%$ (endemic areas with bad sanitation) (COTS)

$0.2 \%$ (endemic areas in open settings - suitable for initial calculations of early resource requirements)

NB Overall, $90 \%$ of cases are mild and difficult to distinguish from other types of D.

NB Asymptomatic carriers are very common (10x \# of cases).

Referral rates for IVs $\quad 20 \%$ of cases (much higher-70\% at ICDDR as it shortens recovery time)

Case fatality ratios $\quad 1 \%$ (with good care)

The following catchment populations will yield 100 acute Pts of whom 20 will be severely dehydrated:

refugee camp of 2000 people (AR of $5 \%=100 \mathrm{Pts}$ ) open settings in endemic area with 50,000 people (AR $0.2 \%=100 \mathrm{Pts}$ )

A population of 100,000 infected individuals in an epidemic area will yield the following (WHO):

Population infected $\quad 100,000$

Clinical cases $\quad 1,000$ ( $1 \%$ of infected population)

Cases needing early resources $200(20 \%$ of cases $)$

Cases needing IV therapy $200(20 \%$ of cases $)$

Anticipated deaths $10(1 \% \mathrm{CFR})$

NB In non-endemic areas, AR adults $>$ AR pedes because adults have higher exposure risks.

In endemic areas, AR pedes $>$ AR adults because adults have been exposed since childhood

\section{Clinical Medicine}

\section{Delivery of Health Services}

Active case finding through CHWs

Treatment facilities -2 types

outpatient ORS center

1. ORS packets, $\mathrm{Zn}$

2. clean water

3. $\mathrm{ABX}$ (in the case of a shigellosis outbreak)

4. recording form for demographic information

5. trained staff (nurse, health aides)

6. soap to give to families (especially in the case of a shigellosis outbreak) 
7. communication (radio, telephone) with the treatment facility for patients who need transfer, and means of transport (ambulance, horse, rickshaw, etc.)

inpatient treatment facility (section of existing hospital/health center or makeshift facility)

1. one way traffic flow-from triage to OPD/home and IPD

2. running water or close proximity to water source

3. large quantities of safe water $(40-60 \mathrm{~L} / \mathrm{p} / \mathrm{d})$

4. adequate waste disposal system

5. shelter from weather

6. recording form for patient demographic information and clinical status

7. IVs, suspension lines, ORS, meds, and other essential supplies in treatment areas

8. trained staff including physicians, nurses, health aides, and cleaners with supervision of Pts 24/7

9. fee waiver policy for emergencies

10. 1 family carer or visitor/Pt

\section{Standardized Case Management}

\section{Suspected cholera outbreak definition}

Pt $>5$ yr develops acute watery diarrhea with severe dehydration or death

any sudden increase in the daily \# of patients with acute watery diar-

Case definitions

rhea especially if they pass typical rice-water stools

cholera

where the disease is not known to be present:

$\mathrm{Pt}>5 \mathrm{yr}$ with acute watery diarrhea plus severe dehydration or death

where the disease is confirmed present:

$\mathrm{Pt}>2 \mathrm{yr}$ with acute watery diarrhea

bacillary dysentery

Pt with acute diarrhea plus visible blood in the stool

Treatment protocols

Overview

assess for dehydration

rehydrate the $\mathrm{Pt}$

maintain hydration by replacing ongoing fluid losses until diarrhea stops

give an oral antibiotic to the Pt with severe dehydration

feed the $\mathrm{Pt}$ 
Triage by dehydration status

$1 / 3$ Pts without dehydration $\rightarrow$ OPD for ORS

2/3 Pts with dehydration $\rightarrow$ SSU for IVF, ORS

$\rightarrow$ IPD for adults with D > 14d, typhoid fever, meningitis; pedes with malnutrition + infectious complications

HPI \& stool appearance help make presumptive Dx

Shigella HPI: $3 \mathrm{~d}$ of Sx with F + abdominal pain/cramps + bloody D (not voluminous) + tenesmus (from colonic mucosal inflammation and erosions), anorexia \pm dehydration.

Stools: chunky style with visible coagulated blood (related to coagulation necrosis in bowel). However, watery diarrhea may appear early in the illness.

Cholera HPI: $6 \mathrm{~h}$ of Sx onset in early morning with V, profuse watery $\mathrm{D}$ without pain or cramps, marked dehydration.

Stools: homogeneous rice water, occasionally turbid with fine particulate, occasionally yellowish when mixed with urine.

Vibrio parahemolyticus

Stools: "meat-washed stool" treated similar to cholera

\section{Rotavirus}

HPI: V without F

Stools: look like mashed pulses

Amoeba Stools: appears dark brown because the parasite oxidizes heme.

IVF ASAP (<1 minute) if severely dehydrated. $75+\%$ of Pts with dehydration at ICDDR get IVs because they feel better more quickly. Otherwise PO sufficient. NGT ok if IV and PO are unsuccessful (very rare).

NB: acetate in cholera saline may cause hypocalcemia and tetany.

ORS for rehydration

$\mathrm{Pt}<6$ months, glucose ORS

$\mathrm{Pt}>6$ months, rice-based ORS

Zn Pt $<6$ months, $10 \mathrm{mg}$ PO qd $\times 10-14 \mathrm{~d}$

Pt $>6$ months to $5 \mathrm{yr}, 20 \mathrm{mg}$ PO qd $\times 10-14 \mathrm{~d}$

$\mathrm{ABX}$ always given under physician supervision avoid prophylactic use

\section{Cholera}

In epidemic season, use ABX x 1 dose with all dehydrated patients with suspected cholera.

In non-epidemic season, use clinical judgment on watery diarrhea. 
If organism is sensitive, doxycycline is generally DOC. ICDDR has used azithro as DOC in all ages.

\section{Dysentery}

Use ABX for dysentery year round (if resources limited, prioritize high risk groups-U5, malnourished, toxic, post-measles, pregnant, old, etc). If organism is sensitive, cipro is generally DOC. ICDDR has used cipro in adults and pivmecillinam in pedes. Ceftriaxone is 2 nd choice for both age groups.

\section{Rotavirus}

No ABX unless $\mathrm{Pt}$ is very ill and clinical picture suggests combined disease.

\section{ETEC}

Usually, no ABX, unless Pt develops severe dehydration.

Misc Sx Vomiting: no antiemetics, but use NGT or IVF if Pt ultimately can't take PO

Fever: \pm antipyretics depending upon resources available Physician rounds q $2 \mathrm{~h}$ on severely ill $\mathrm{Pts} ; 4 \mathrm{x} / \mathrm{d}$ on Pts with some dehydration (SSU); $2 \mathrm{x} / \mathrm{d}$ on $\mathrm{Pts}$ without dehydration (OPD).

\section{Disposition}

Cholera improves quickly - young Pts get off IVF by 24 h, old Pts by $48 \mathrm{~h}$.

ETEC lasts for 3-5 d.

Shigella dysentery lasts for 3-5 d if uncomplicated.

Rotavirus lasts for $5 \mathrm{~d}$ until kids can feed again (strips off brush border). Maternal motivation is important during this time.

Overall, rice-based ORS ends up adequately treating $85 \%$ of diarrheas.

Discharge

no dehydration, $<$ purging, able to take ORS adequately

$5-7 \%$ return visits are expected; $>10 \%$ return visits mean discharge policy is too aggressive

\section{Essential drugs \& supplies}

- cholera kits

- drug choices driven by culture data (10-20 specs)

$\underline{\text { Referral guidelines }}$

- refer all Pts refractory to rehydration and standard ABX to hospital for definitive care

$\underline{\text { Secondary prevention for the patient }}$

- ORS liberally distributed with instructions for use-2 ORS sachets to all discharged Pts (home based salt solution is dangerous)

- soap distribution to accompanying persons

Secondary prevention at the site

- isolate the affected $\mathrm{Pt} / \mathrm{ward} / \mathrm{camp} /$ defecation area.

- impose hand washing upon entry/exit to affected hut/clinic/camp/defecation area, etc $(0.05 \% \mathrm{Cl})$. 
in-service staff-HCWs should not handle food or water (ORS); kitchen staff should not handle hospital waste.

- recruit cleaners, establish cleaning routine $(0.5 \% \mathrm{Cl})$.

wash spills ASAP

wash floor q $4 \mathrm{~h}$

collect stool collection buckets bd or PRN (wash them with $2.0 \% \mathrm{Cl}$ )

change and wash cot covers qd

wash cots after each $\mathrm{Pt}$

spray the affected area qd (water storage containers, water distribution points, latrines, door handles, etc in clinic, camp, and defecation area)

- identify body fluid disposal site $(2.0 \% \mathrm{Cl})$.

hold $\mathrm{Cl}$ treated fluids $2 \mathrm{~h}$ before dumping

- establish hygienic funereal practices $(2.0 \% \mathrm{Cl})$

\section{Epidemic Management}

Outbreak investigation protocol in place

- rapid response teams to investigate case reports

- epidemic investigation kits to mobilize

- specimens to collect

- labs to confirm Dx of $V$. cholerae, $S$. dysenteriae, other shigella, and E. coli $\mathrm{O} 157: \mathrm{H} 7$

dipstick identification on representative sample of specs is useful for cholera, but $\mathrm{C} \& \mathrm{~S}$ is essential because dipsticks are not available for shigella, ETEC. Vibrio are hardy if kept moist and cool. They can survive a week in Cary Blair media. Shigella are fragile and difficult to recover if transport time $>1 \mathrm{~d}$.

$5-10$ isolates initially to confirm outbreak

30-50 isolates initially to create ABX use policy (bacterial resistance renders cotrimoxazole, amp/amox, nalidixic acid, and tetracycline unusable)

20-30 isolates monthly from IPD and OPD before ABX therapy to assess evolving $\mathrm{ABX}$ resistance

10-20 isolates periodically to reference laboratory to confirm ABX resistance patterns and undertake molecular studies

20 isolates at end of the outbreak to confirm that new diarrheas are not epidemic pathogens

NB Systematic sampling is most representative - eg every 10th Pt or all Pts q 2 weeks adjusted as needed to collect the necessary specs.

Sensitivity $>>$ important than specificity in RDT screening during an epidemic. 
Pts from one geographic area are more likely to constitute a cluster involving a new pathogen.

An area may be considered cholera-free after 2 incubation periods (total of $10 \mathrm{~d}$ ) have passed without cholera disease. However, hospital monitoring should continue for a year due to tendency of enteric pathogens to re-emerge long after they are declared gone.

Cholera may be viable but nonculturable from the environment; environmental monitoring has many false negatives. consider improvements to existing diagnostic labs

- hotline set up for reporting of rumor

Health reference and educational materials in place

- case definitions

- case management and referral guidelines for communicable diseases with epidemic potential

- Pt, provider, and community educational materials

- specimen handling protocols

Epidemic command \& control center established

- principles and practices of incident mgmt

- unified command of multi-disciplinary specialists

- information channel to government and stakeholders

- support by government for technical actions

Coordination with technical sectors - particularly WASH (CFR is a function of case mgmt, but AR overall is a function of WASH)

- water supply, purification, and distribution systems

bucket chlorination is low tech but reasonable way to reach individual $\mathrm{HH}$ or small communities

water treatment units need Ca hypochlorite, chlorimetric, and colimetric monitoring devices

chlorinators worth considering at water sources of high public demand and epidemic activity

- hygiene promoters with environmental health assessors to address hand and food hygiene in communities around the outbreak area (think ring vaccination with knowledge)safe disposal of medical waste and infectious sludge from treatment facilities

Medical logistics - resource prepositioning and stockpiles

- cots (take one and have carpenter make copies)

- plastic sheets with defecation hole or sleeve

- buckets (white color for stool-enables recognition of diarrhea color; different color for emesis; different color for domestic waste)

- IVF, IV sets, IV poles or suspension cords (cholera kits)

- ORS powder and ORS vats (> 50 L)

- cups, spoons

- NGTs, syringes

- soap, cleaning supplies

- bleaching powder (eg $\mathrm{Ca}$ hypochlorite)-chlorinate water sources where feasible, otherwise bucket chlorinate at untreated sources

- equipment (eg chlorimetric and colimetric monitors) 
Information management

- update local authorities

- intensify disease surveillance (health authorities, WHO)

- issue health advisories (health \& political authorities)

- reinforce training of public health cadres on diarrhea prevention and control

- reinforce education of clinicians on diarrhea case management and secondary prevention

- initiate public awareness and health education campaign

health messages content (WHO Cholera manual or COTS card for Health Promotion Worker)

health messages dissemination-printed, loudspeaker, broadcast, community groups

health messages uptake-community understands hand washing, ORS use, and 2 prevention measures

$\underline{\mathrm{OCV}}$

- Cholera epidemic brings huge political pressures to DO something. This often translates into a hastily conceived vaccination campaign that distracts from core principles of cholera management. For every symptomatic $\mathrm{Pt}$, there may be 90 asymptomatic carriers. In an established epidemic, the affected community is already extensively infected. Cholera vaccination, under these circumstances, has little public health benefit for the resource investment. If undertaken, the following will apply:

- Vaccination campaign requires numerous staff. Community mobilizers are key. Clinical staff should not be poached from their clinical duties. Supervisors must be free to move at will.

- Logistics is key - if the 1st day goes badly, the campaign goes badly.

- Mark the domiciles which are done.

- Hold after-action meetings each day.

- Last day, use mobilizers with mobile broadcasting to attract those who missed out.

- Second phase vaccination should include CHWs with multi-purpose messages on water and sanitation.

\section{Key lessons in epidemic}

Avoid: press exaggeration

ABX prophylaxis

reliance on IVF and insufficient ORS

lab investigation of cases once epidemic etiology is ascertained prolonged hospitalization

hospital discharge criteria requiring multiple negative stool cultures enthusiasm for OCV during epidemic exaggerated water purification objectives

concentration of technical competencies in $\mathrm{MOH}$ at expense of districts

failure to share information with stakeholders 


\section{References}

1. World Health Organization. Cholera vaccines: WHO position paper. Weekly Epidemiological Record 26 March 2010; 85(13):117-28.

2. International Centre for Diarrhoeal Disease Research, Bangladesh, and Swiss Tropical Institute (eds). Cholera outbreak training and shigellosis (COTS) program [CD-ROM version 1.0, undated]. Available from: International Centre for Diarrhoeal Disease Research, Bangladesh, and http://www.cotsprogram.com. 


\section{INFLUENZA}

\section{Pathogens}

Influenza viruses comprise 3 genera-influenza types $\mathrm{A}, \mathrm{B}$, and $\mathrm{C}-$ each with 1 species.

- Influenza type A is divided into subtypes based upon serological response to hemagglutinin (HA) and neuraminidase (NA) glycoproteins. There are 16 different HA subtypes and 9 different NA subtypes. H1N1, $\mathrm{H} 2 \mathrm{~N} 2$, and $\mathrm{H} 3 \mathrm{~N} 2$ are responsible for the major human pandemics in the last century. H2N2 virus circulated between 1957 and 1968 but currently does not. Only influenza A subtypes infect birds, and all subtypes can do so. Bird flu viruses do not usually infect humans. But, in 1997, an outbreak of H5N1 avian influenza in poultry in Hong Kong marked the first known direct human transmission of avian influenza virus from birds to humans. Since then, H5, H7, and H9 avian influenza subtypes have been shown to infect humans.

- Influenza type B is morphologically similar to A and also creates seasonal and epidemic disease.

- Influenza type $\mathrm{C}$ is rare but can cause local epidemics.

Seasonal human influenza vaccine currently has 3 strains - H1N1/H3N2/B.

Influenza disease in humans has a short incubation period (1-3 d). Early symptoms are non-specific. It is highly infectious, especially early in the course of the disease, with a large \# of asymptomatic carriers. Transmission potential $\left(\mathrm{R}_{0}\right)$ is a function of infectivity, period of contagiousness, daily contact rate, and host immunity. In general, the faster the transmission, the less feasible is interrupting transmission thru usual disease control tools of case finding, isolation, contact tracing, and ring vaccination.

Table 7.6.3

$\mathbf{R}_{0}$ of Different Diseases (adapted from CDC [1])

\begin{tabular}{|l|l|}
\hline \multicolumn{1}{|c|}{ Disease } & \multicolumn{1}{c|}{$\mathbf{R}_{\mathbf{0}}$} \\
\hline Influenza pandemic & $2-3(1918)$ \\
\hline Influenza seasonal & $1.5-3$ \\
\hline Measles & $12-18$ \\
\hline Polio & $5-7$ \\
\hline Rubella & $6-7$ \\
\hline Diphtheria & $6-7$ \\
\hline Smallpox & $5-7$ \\
\hline Mumps & $4-7$ \\
\hline SARS & 3 (excluding superspreaders) \\
\hline
\end{tabular}


Table 7.6.4

Disease Comparison (adapted from US DHHS [2])

\begin{tabular}{|c|c|c|c|}
\hline \multirow[t]{2}{*}{ Attribute } & \multicolumn{3}{|c|}{ Disease } \\
\hline & Influenza & Smallpox & SARS \\
\hline Transmissibility & High & Medium & Low \\
\hline $\mathrm{R}_{0}$ & $\begin{array}{l}>2 \text { (estimated } 1.8 \\
\text { for } 1918 \text { pandemic) }\end{array}$ & $5-7$ & $\begin{array}{l}3 \text { (excluding } \\
\text { superspreaders) }\end{array}$ \\
\hline Geography & $\begin{array}{l}\text { Widespread, multi- } \\
\text { focal epidemics }\end{array}$ & & Focal epidemics \\
\hline $\begin{array}{l}\text { Transmission } \\
\text { location }\end{array}$ & Community & & $\begin{array}{l}\text { Within families and } \\
\text { health care settings }\end{array}$ \\
\hline Incubation period & 1-3 days & $10-12$ days $(\mathrm{CDC})$ & $7-10$ days \\
\hline Attack rate & $10-35 \%$ & $58 \%(\mathrm{CDC})$ & low \\
\hline CFR & $\begin{array}{l}1 \% \text { (seasonal) } 50 \% \\
\text { (highly pathogenic } \\
\text { avian influenza) }\end{array}$ & $\begin{array}{l}50 \% \\
\text { (unvaccinated) } \\
30 \% \text { (vaccinated } \\
\text { post exposure) } \\
11 \% \text { (> } 20 \text { y before } \\
\text { exposure) } 1.4 \% \\
\text { (0-10 y before } \\
\text { exposure) }\end{array}$ & $5-10 \%$ \\
\hline $\begin{array}{l}\text { Epidemic } \\
\text { investigation }\end{array}$ & $\begin{array}{l}\text { Unlikely to track } \\
\text { spread based upon } \\
\text { movement of } \\
\text { infected persons or } \\
\text { contacts }\end{array}$ & $\begin{array}{l}\text { High ability to } \\
\text { track spread based } \\
\text { upon movement of } \\
\text { infected persons or } \\
\text { contacts }\end{array}$ & $\begin{array}{l}\text { Potential ability to } \\
\text { track spread based } \\
\text { upon movement of } \\
\text { infected persons or } \\
\text { contacts }\end{array}$ \\
\hline $\begin{array}{l}\text { Interventions } \\
\text { to prevent } \\
\text { transmission }\end{array}$ & $\begin{array}{l}\text { International } \\
\text { travel restrictions/ } \\
\text { screening possible } \\
\text { but unlikely to } \\
\text { prevent pandemic; } \\
\text { quarantine unlikely; } \\
\text { school closing and } \\
\text { limits on public } \\
\text { events likely; } \\
\text { vaccination of } \\
\text { priority groups }\end{array}$ & $\begin{array}{l}\text { Vaccination } \\
\text { effective if given } \\
\text { within } 4 \mathrm{~d} \text { of } \\
\text { infection }\end{array}$ & $\begin{array}{l}\text { International } \\
\text { travel restrictions/ } \\
\text { screening possible } \\
\text { and likely to help } \\
\text { prevent outbreaks; } \\
\text { quarantine likely; } \\
\text { school closing and } \\
\text { limits on public } \\
\text { events unlikely }\end{array}$ \\
\hline $\begin{array}{l}\text { Disruption of } \\
\text { transportation } \\
\text { infrastructure, } \\
\text { community } \\
\text { services }\end{array}$ & $\begin{array}{l}\text { Widespread, } \\
\text { widespread }\end{array}$ & & $\begin{array}{l}\text { Widespread, } \\
\text { little }\end{array}$ \\
\hline
\end{tabular}




\section{Preventive Medicine}

Vaccination when available, social distancing, cough etiquette, hand hygiene

\section{Epidemiological Surveillance}

Rapid case investigation, contact tracing, and clusters containment Quick reporting of suspected cases by affected countries

\section{Clinical Medicine}

\section{Delivery of Health Services}

Passive case finding but use of influenza clinics and fever hospitals

\section{Standardized Case Management}

\section{Case definitions}

\section{Treatment protocols}

technical expertise in case management including rational use of antiviral treatment and prophylaxis

\section{Essential drugs}

monitoring for counterfeit antiviral medications

Referral guidelines

what to do in resource poor settings

Secondary prevention

- health care setting

$\mathrm{Pt}$ isolation in specific hospital/ward designations

private room or cohorting with other flu Pts

minimize transport of $\mathrm{Pt}$ outside room

limit \# of HCWs interacting with flu Pt

limit \# of visitors interacting with flu Pt

infection control

standard standard precautions with PPE and hand washing contact hand hygiene

airborne powered air purifying or N95 respirators, cough suppression

droplet N95 respirators

- home setting

voluntary Pt isolation in private room

minimize transport of Pt outside room

limit \# of family interacting with flu Pt

infection control

standard standard precautions with PPE and hand washing 
contact hand hygiene

airborne N95 respirators, cough suppression

droplet N95 respirators

voluntary quarantine of contacts of known cases

NB management of contacts (contact tracing, contact monitoring) potentially useful only very early in epidemic

quarantine of close contacts for a complete incubation period potentially useful only very early in epidemic

- all settings - try to decrease potential for infection

vaccination

seasonal or post-exposure antiviral chemoprophylaxis

$<$ susceptibility to infection by $30 \%$

if infection occurs, < infectiousness by $36 \%$

if disease occurs, $<$ probability of clinically recognizable Sx by $65 \%$

\section{Epidemic Management}

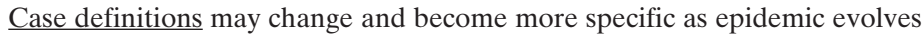

Case management guidelines

Outbreak management protocol

- rapid response teams to investigate case reports

- epidemic investigation kits to mobilize

- specimens to collect

- labs to verify diagnosis and share specimens with peer labs

- contacts to trace and? quarantine

- hotline use and rumor investigation

Secondary prevention

- specific groups of exposed or at risk in the community-most likely to work when there is limited disease transmission in the area, most cases can be traced to a specific contact or setting, and intervention is considered likely to slow the spread of disease

eg quarantine of groups of people at known common source exposure (airplane, school, workplace, hospital, public gathering; ensure delivery of medical care, food, and social services to persons in quarantine with special attention to vulnerable groups) (useless once there is community-based spread)

eg containment measures at specific sites or buildings of disease exposure (focused measures to $>$ social distance)

cancel public events (concerts, sports, movies)

close buildings (recreational facilities, youth clubs)

restrict access to certain sites or buildings

- community-wide measures (affecting exposed and non-exposed)most likely to work where there is moderate to extensive disease transmission in the area, many cases cannot be traced, cases are increasing, and there is delay between Sx onset and case isolation. 
infection control measures

ARI etiquette-cover nose/mouth during cough or sneeze, use tissues, wash hands

avoidance of public gatherings by persons at high risk of complications

NB use of masks by well persons is not recommended "snow" (stay-at-home) days and self-shielding (reverse quarantine) for initial $10 \mathrm{~d}$ period of community outbreak - may reduce transmission without explicit activity restrictions

closure of schools, offices, large group gatherings, public transport (pedes more likely to transmit disease than adults)

NB community quarantine (cordon sanitaire)-restriction of travel in and out of an area is unlikely to prevent introduction or spread of disease

- international travel

NB travel advisories to restrict international travel are generally useless in slowing epidemic spread

NB health screening for fever and respiratory Sx at ports of entry is also generally useless in slowing epidemic spread

Resource prepositioning, stockpiles, and supply chain management

- facemasks

- PPE

- vaccines, antiviral drugs

Information management

- health workers

- political authorities

- public via awareness campaign and behavior change communication

Contingency planning

- incident/event management system and role designations

- surveillance, investigation, and containment

- vaccines, antivirals

- provision of essential services (lifelines, health care, and emergency response)

- culturally appropriate corpse management

\section{Avian Influenza}

\section{Poultry Cases}

- Biosecurity

- Cull

- Disposal

- Disinfection

- Control movement

- Quarantine

- Count (surveillance around affected flocks)

- Ring vaccination 
Overall approach

primary prevention: animal vaccination (prevents viral reassortment)

cases: biosecurity (of premises), cull, disposal (of carcasses), disinfection

(of premises)

contacts: quarantine, control movement, count (surveillance), ring vaccination

\section{Human Cases}

- Health Care-infection control (PPE)

- Control movement

- Quarantine

- Count (surveillance)

- Vaccines

- Antiviral drugs

Overall approach

primary prevention: animal vaccination (prevents viral reassortment)

cases: isolation, infection control, antiviral therapy

contacts: quarantine, infection control, count (surveillance), vaccination, antivirals with chemoprophylaxis for high risk persons

NB: travel screening \& restrictions, quarantine, and school cancellation are not effective measures.

\section{References}

1. World Health Organization and Centers for Disease Control and Prevention. History and epidemiology of global smallpox eradication. In: Smallpox: Disease, Prevention, and Intervention training course, undated. Available from: https://emergency.cdc.gov/agent/smallpox/training/overview/pdf/eradicationhistory.pdf .

2. US Department of Health and Human Services. Pandemic influenza preparedness and response plan draft. Annex 12, p 8-11. August 2004. Available from: US Department of Health and Human Services, Washington, DC. 


\section{MALARIA}

\section{Pathogen Vectors}

Table 7.6.5

Mosquito Biology [1]

\begin{tabular}{|c|c|c|c|c|c|c|}
\hline $\begin{array}{l}\text { Vector } \\
\text { group }\end{array}$ & Species & $\begin{array}{l}\text { Breeding } \\
\text { sites }\end{array}$ & $\begin{array}{c}\text { Resting } \\
\text { sites }\end{array}$ & $\begin{array}{c}\text { Transmission } \\
\text { activity }\end{array}$ & $\begin{array}{l}\text { Blood } \\
\text { source }\end{array}$ & Diseases \\
\hline Anophelines & Anopheles & $\begin{array}{l}\text { Natural } \\
\text { pools of } \\
\text { unpolluted } \\
\text { water }\end{array}$ & $\begin{array}{l}\text { Indoor/ } \\
\text { outdoor }\end{array}$ & $\begin{array}{l}\text { Evening and } \\
\text { night }\end{array}$ & $\begin{array}{l}\text { Humans } \\
\text { and } \\
\text { animals }\end{array}$ & $\begin{array}{l}\text { Malaria, } \\
\text { filariasis } \\
\text { arboviruses }\end{array}$ \\
\hline \multirow[t]{3}{*}{ Culicines } & Aedes & $\begin{array}{l}\text { Water } \\
\text { containers } \\
\text { (tires), } \\
\text { pools of } \\
\text { stagnant } \\
\text { water }\end{array}$ & $\begin{array}{l}\text { Indoor/ } \\
\text { outdoor }\end{array}$ & Day & $\begin{array}{l}\text { Humans } \\
\text { and } \\
\text { animals }\end{array}$ & $\begin{array}{l}\text { Filariasis, } \\
\text { YF, DF }\end{array}$ \\
\hline & Culex & $\begin{array}{l}\text { Organically } \\
\text { polluted } \\
\text { water } \\
\text { (sewers), } \\
\text { natural } \\
\text { pools of } \\
\text { unpolluted } \\
\text { water }\end{array}$ & $\begin{array}{l}\text { Indoor/ } \\
\text { outdoor }\end{array}$ & $\begin{array}{l}\text { Day and } \\
\text { night }\end{array}$ & $\begin{array}{l}\text { Humans } \\
\text { and } \\
\text { animals }\end{array}$ & $\begin{array}{l}\text { Filariasis, } \\
\text { viral } \\
\text { encephalitis }\end{array}$ \\
\hline & Mansonia & $\begin{array}{l}\text { Unpolluted } \\
\text { water with } \\
\text { plants }\end{array}$ & $\begin{array}{l}\text { Indoor/ } \\
\text { outdoor }\end{array}$ & $\begin{array}{l}\text { Day and } \\
\text { night }\end{array}$ & $\begin{array}{l}\text { Humans } \\
\text { and } \\
\text { animals }\end{array}$ & Filariasis \\
\hline
\end{tabular}

Source: M Connolly (ed) in Communicable disease control in emergencies - a field manual $\odot 2005$. Used with permission of the World Health Organization.

\section{Anopheles vector biology}

egg becomes adult mosquito in $9 \mathrm{~d}$

adult mosquito becomes infective in $12 \mathrm{~d}$ after bite on infected host susceptible human host becomes infective in $9 \mathrm{~d}$ after bite from infected mosquito

:. earliest human clinical disease in $30 \mathrm{~d}$ after eggs are laid

\section{Preventive Medicine}

\section{Health messages}

Follow the 4-D rule:

dusk and dawn stay indoors as much as possible with window screens in good repair

dress in light colored long sleeve shirts and long pants when outside 
DEET (N,N-diethyl-M-toluamide) based mosquito repellants

drain any standing water from the area (flower pots, old tires, clogged rain gutters, etc); flush troughs, birdbaths, wading pools,

$\underline{\text { Insecticides }}$ etc every 3 days

$10 \%$ DDT, $1 \%$ malathion, $1 \%$ permethrin

insecticide treated nets (ITNs) (esp long-lasting nets with duration of 3-5 years)

indoor residual spraying (IRS) (esp within 2 weeks of high transmission season; more effective in Asia than Africa; not appropriate for dengue)

deterrence:

\# mosquitoes which don't enter room

repellence:

\# mosquitoes which enter then leave room

bite inhibition:

\# mosquitoes which enter room but don't bite

direct knock down: \# mosquitoes which are knocked down, but still live

direct death: \# mosquitoes killed

NB aerial spraying \& outdoor spraying (fogging) are not especially useful in malaria

deterrence \& repellence effectiveness is species-specific (eg aedes > anopheles $>$ culex)

coils work by knockdown effect, not bite inhibition

Larvicides

organophosphate (Temephos-safe for drinking water)

Bacillus thuringiensis israelensis (bacterial toxin)

Mesocyclops copepods (used in Vietnam in village water tanks)

larvivorous fish (used in China in domestic water tanks)

Insect repellents (useful for individuals who can afford it but not as public health intervention)

DEET

Chemoprophylaxis

seasonal malaria chemoprophylaxis (SMC)

international staff travelling to endemic areas

intermittent preventive treatment (IPT) for pregnant women in endemic areas where continuous chemoprophylaxis is not feasible

Generally, control programs rely upon:

3 major components - ITN, IPT, and prompt clinical treatment

2 ancillary components - IRS, environmental clean-up of breeding sites

\section{Epidemiological Surveillance}

Geographic reconnaissance

mapping of target area before spraying

mapping of homes for IRS

Entomological survey 
night mosquito survey

\# mosquitoes trapped/hr (calculation of man-biting rate)

species identification

mosquito dissection (F only) to determine parity; \% parity inversely related to spraying effectiveness

Malariometric survey

prevalence in sample of 100 persons

spleen rate in sample of 100 persons

slide positivity rate (ratio of \# confirmed cases/\# clinically suspected cases)

proportion of fever caused by malaria (\# fever cases with confirmed parasitemia/\# total fevers)

Malaria mortality (\# malaria deaths/10,000 p/d for given area)

Case fatality rate in all malaria cases

Case fatality rate in severe malaria cases

Proportional mortality (\# malaria deaths/\# total deaths)

Malaria incidence rate

\section{Clinical Medicine}

\section{Delivery of Health Services}

Active case finding through $\mathrm{CHWs}$

\section{Standardized Case Management}

Case definition

Pt with F or history of F associated with Sx such as N, V, D, headache, back pain, chills, myalgia, where other infectious diseases have been clinically excluded

Rapid (point of care) diagnostic testing (RDT)

Treatment protocols per local health authorities

Essential drugs

Referral guidelines

Secondary prevention

community education

health messages-printed, loudspeaker, broadcast, community groups

community understands transmission and prevention measures

\section{Epidemic Management}

Outbreak management protocol

Secondary prevention

Resource prepositioning (stockpiles \& supply chain management) 
indoor residual spraying (IRS) (within 2 weeks of epidemic)

mass fever treatment (MFT) (active case finding \& fever treatment with antimalarials)

mass drug administration (MDA) (cover $80 \%$ of population within 2 weeks)

Information management

Contingency planning

\section{Reference}

1. Connolly M (ed). Communicable disease control in emergencies - a field manual. Geneva: World Health Organization, 2005. 


\section{MEASLES}

\section{Preventive Medicine}

\section{EPI}

Subnational immunization days

Measles immunization of contacts of confirmed case if contacts have $<2$ doses of vaccine

\section{Epidemiological Surveillance}

Surveillance definition becomes clinical once outbreak is lab-confirmed

\section{Clinical Medicine}

\section{Delivery of Health Services}

Active case finding through $\mathrm{CHWs}$

\section{Standardized Case Management}

Case definition

An illness characterized by all of the following clinical features: a generalized rash lasting greater than or equal to $3 \mathrm{~d}$ (exanthems generally without symmetry or pruritis); $\mathrm{T} \geq 38.3{ }^{\circ} \mathrm{C}\left(101{ }^{\circ} \mathrm{F}\right)$; cough, or coryza, or conjunctivitis

Treatment protocols emphasizing supportive care and treatment of complications

Vitamin A

Table 7.6.6 Vitamin A Treatment Schedules in Infant/Child Deficiency States

\begin{tabular}{|l|c|r|}
\hline \multicolumn{1}{|c|}{ Age } & Initial Dose & Next Day Dose \\
\hline $0-6$ months & $50,000 \mathrm{IU}$ & $50,000 \mathrm{IU}$ \\
\hline $6-11$ months & $100,000 \mathrm{IU}$ & $100,000 \mathrm{IU}$ \\
\hline $1+$ years & $200,000 \mathrm{IU}$ & $200,000 \mathrm{IU}$ \\
\hline
\end{tabular}

Essential drugs

Referral guidelines

Secondary prevention

community education

health messages-printed, loudspeaker, broadcast, community groups

community understands measles transmission and prevention measures 


\section{7. Technical Annexes}

\section{Epidemic Management}

Identify cause of the outbreak Undertake vaccination campaign

Strengthen routine immunization and surveillance

Table 7.6.7

Population Vaccination Status and Contributing Causes

\begin{tabular}{|l|l|}
\hline \multicolumn{1}{|c|}{ Vaccination Status and Patient Age } & \multicolumn{1}{|c|}{ Probable Cause } \\
\hline$>50 \%$ cases U5 \& unvaccinated & low coverage \\
\hline$>50 \%$ cases U5 \& vaccinated & not measles, or vaccine ineffective \\
\hline$>50 \%$ cases O5 in high coverage area & disease shift to older age group \\
\hline high $\%$ of cases in infants & low routine coverage \\
\hline
\end{tabular}




\section{MENINGITIS}

\section{Pathogens and Epidemiology}

Meningitis is a disease with significant mortality. Meningococcus (Neisseria meningitides) is renown for its rapid onset, rapid progression (death sometimes within hours), and high mortality (50\% untreated).

There are 13 serogroups of Neisseria meningitides but only 6 (A, B, C, W, X, Y) are known to cause epidemics.

The bacteria spread from person to person via respiratory and nasal secretions. Kissing, sharing eating and drinking utensils, cigarettes, coughing, and sneezing are recognized methods of transmission. Close contacts over a period of time, as between household or dormitory residents, are most commonly affected. Population movements (eg pilgrimages, displacement, military recruitment), poor living conditions, and overcrowding are epidemic risk factors.

Large, recurring epidemics of meningitis occur in the "meningitis belt" of sub-Saharan Africa where over 430 million people live. This belt encompasses 26 countries from Senegal in the west to Ethiopia in the east and as far south as Tanzania and the Democratic Republic of Congo. Subsaharan Arica has epidemic seasonality. Dry seasons and droughts favor epidemics. Rains stop them. Large regional epidemics, as well as epidemics in displaced populations and refugee camps, have mainly been due to meningococcus serogroup A. Since 2010, extensive use of meningococcal type A conjugate vaccine in the meningitis belt has reduced the incidence and case load of type A epidemics by nearly $60 \%$. In 2016, the most common lab confirmed meningitis isolate was Streptococcus pneumoniae.

In non-epidemic settings, Neisseria.meningitidis, Streptococcus pneumoniae, and Haemophilus influenzae account for $80 \%$ of all cases of bacterial meningitis. Prior to the availability of conjugate vaccines, $H$. influenza type b (Hib) was the most common cause of childhood bacterial meningitis outside of epidemics. Where Hib vaccines are in the routine infant immunization schedule, Hib meningitis has nearly disappeared.

\section{Preventive Medicine}

Polysaccharide vaccines are available with 2 serotypes (A and C), 3 serotypes (A, C, and W) or 4 serotypes (A, C, W, and Y). Duration of immunity is approximately 3 years.

Meningococcal protein conjugate vaccines confer longer immunity but at higher cost than polysaccharide vaccines. Monovalent conjugate vaccine against group C dates from 1999, and tetravalent (A, C, W, and Y) conjugate vaccine dates from 2005. Group B vaccine made from 4 bacterial proteins has been licensed since 2014 but is not readily available.

Meningococcal vaccines have a very low incidence of side effects. 


\section{Epidemiological Surveillance}

Regular disease surveillance is necessary to detect outbreaks. The epidemic threshold is 10 suspected cases/ 100,000 population in any given week. Two suspected cases of meningitis in the same settlement should trigger an outbreak investigation.

Nasopharyngeal carriage rates do not predict epidemics.

\section{Clinical Medicine}

$80-85 \%$ of meningococcal disease presents with meningitis. $80 \%$ of cases occur in patients $<30 \mathrm{y} / \mathrm{o}$. Peak incidence in meningitis belt is ages 5-10 yrs. Diagnosis is straightforward when patient presents with signs of meningitis-fever, headache, vomiting, changes in mental status. However, most patients have non-specific illness 1-3 days before onset of meningitis. CFR of untreated meningococcal meningitis can be $50 \%$. CFR of properly treated meningococcal meningitis is $<1 \%$.

$15-20 \%$ of meningococcal disease presents with septicemia unaccompanied by meningitis or other focal features. It is a dramatic illness which affects previously healthy children and young adults. It presents with acute fever leading to purpura fulminans (hemorrhagic or purpuric rash), shock, and Waterhouse-Friderichsen syndrome (acute adrenal failure). Etiologic diagnosis can be easily missed. CFR of meningococcal septicemia is $50 \%$ and may be $25 \%$ even with proper treatment.

Diagnosis may be confirmed by agglutination tests, polymerase chain reaction, culture and sensitivity testing of spinal fluid and blood. In many situations, these tests are not available. Throat swabs may be helpful on occasions. Do not delay treatment for tests or test results. Minutes count. It is more important to have a live patient without a confirmed diagnosis than a dead one with a diagnosis.

Differential diagnosis in a tropical patient with fever and altered mental status, but without purpura or shock, includes cerebral malaria. Co-infection may occur.

Standardized case management of bacterial meningitis in developed countries involves 7-10 days of parenteral antibiotic therapy. Drug of choice in adults and older children is ceftriaxone which also rapidly eliminates the carrier state. Alternate drugs include ampicillin and benzylpenicillin which do not eliminate the carrier state. In developing countries, 4 days of parenteral antibiotic therapy are empirically shown to be effective. In large epidemics in resource-poor settings, a single IM dose of chloramphenicol in oil is the drug of choice. For patients who do not improve in 48 hours, a repeat dose may be given.

Viral meningitis is rarely serious and requires only supportive care, Recovery is usually complete. 
Patient isolation and disinfection of the room, clothing, or bedding are not necessary. Respiratory precautions are advised particularly early in the course of treatment.

Chemoprophylaxis of contacts is available in some settings but rarely in the disaster setting. Vigilance and education of close contacts is mandatory.

\section{Epidemic Management}

Epidemic preparedness and early detection of outbreaks are key.

Vaccines against $N$. meningitides serogroups A, C, Y and W135 are very effective in controlling epidemics. In epidemic settings, children 2-10 are the priority target with serogroups $\mathrm{A}$ and $\mathrm{C}$ typically the priority antigens. Rapid mass vaccination campaigns can contain outbreaks in 2-3 weeks. For immunocompetent patients over 2 years, vaccine efficacy rate is $90 \%$ one week after injection. However, duration of immunity may be as little as 2 years in younger children. In some countries, vaccine may also be used with close contacts of sporadic disease cases to prevent secondary cases.

Chemoprophylaxis of contacts is not recommended in epidemics, but community education and ready access to health care are essential. 


\section{VIRAL HEMORRHAGIC FEVER}

\section{Preventive Medicine [1]}

Source control/reduction/elimination

Undertake quarantine and culling of sick reservoir animals and known disease carrier species.

Avoid unnecessary contact with or consumption of dead reservoir animals or known disease carrier species.

Avoid unnecessary contact with suspected reservoir animals and known disease carrier species (eg primates).

Avoid direct or close contact with symptomatic patients.

Establish appropriate communicable disease controls for burial of the dead. Administrative controls (improve people's work practices)

Environmental and engineering controls (isolate people from the hazard)

Avoid needle stick exposure to blood specimens thru automated machine handling.

PPE (protect people with PPE)

Use standard precautions-gloves, masks, and protective clothing-if handling infected animals or patients.

Wash hands after visiting sick patients.

\section{Epidemiological Surveillance}

Active surveillance and contact tracing (enhanced surveillance) through community-based mobile teams

\section{Clinical Medicine [2]}

\section{Delivery of Health Services}

Active case finding (screening and triage) and contact tracing

Dedicated isolation facility

Food provision to isolated patients so they are not dependent on family

\section{Standardized Case Management}

Case definition

Treatment protocols emphasizing supportive care and treatment of complications

Essential drugs

Referral guidelines

Secondary prevention

barrier nursing strictly enforced

family and community education 


\section{Epidemic Management}

Ministerial task force to address policy

Local health authority task force to address procedures

$\underline{\text { National level task forces to comprise }}$

- Response unit

epidemiology (case finding, contact tracing, monitoring, surveillance)

facilities preparation (build beds)

case management

laboratory

infection prevention and control (IPC) (including safe burial)

social mobilization, communication

training

- Support unit (manage resources)

program \& HR planning (16 positions)

staffing model

1. social $/ \mathrm{medical} / \mathrm{cultural}$ anthropologist

2. social mobilization/community engagement specialist

3. social mobilization coordinator at national level

4. epidemiologist

5. IPC specialist

6. $\mathrm{PH}$ advisor

7. subnational field coordinator

8. VHF lab specialist

9. case management specialist

10. EMTs

11. occupational health and safety specialist

12. logistics

13. exit screening

14. data management

15. health communications

16. finance

- Research unit

new therapeutics

new tests

clinical trials

analytic epi and modelling

- Long term measures

health systems recovery

Outbreak management in the field focuses on

- alert verification

- disease surveillance (not relying exclusively or even largely on health facility reporting)

- burial $<48$ hrs after death

- spot checking of contact tracing effectiveness ( $<5$ is inadequate; $25-50$ is reasonable; 75 is not extreme) 


\section{7. Technical Annexes}

- door-to-door community education campaigns

- quarantine sustainment at HH level

NB it's difficult to separate ebola from non-ebola fevers at onset, so holding centers can amplify transmission

Secondary prevention

Resource prepositioning (stockpiles \& supply chain management)

Information management - refute travel and trade restrictions

Community education and social mobilization

- health messages - printed, loudspeaker, broadcast, community groups

- community understands disease transmission and prevention measures

Contingency planning

\section{References}

1. World Health Organization. Ebola: technical guidance documents for medical staff. Available from: https://www.who.int/csr/disease/ebola/protective-measures-staff/en/. Accessed January 2019.

2. World Health Organization. Manual for the care and management of patients in ebola care units/community care centers-interim emergency guidance. WHO/ EVD/Manual/ECU/15.1. Geneva: World Health Organization; January 2015. Available from: https://www.who.int/csr/resources/publications/ebola/ebola-preparedness-checklist/en/. Accessed January 2018. 


\section{Document 7.7 \\ DIAGNOSTIC LABORATORY}

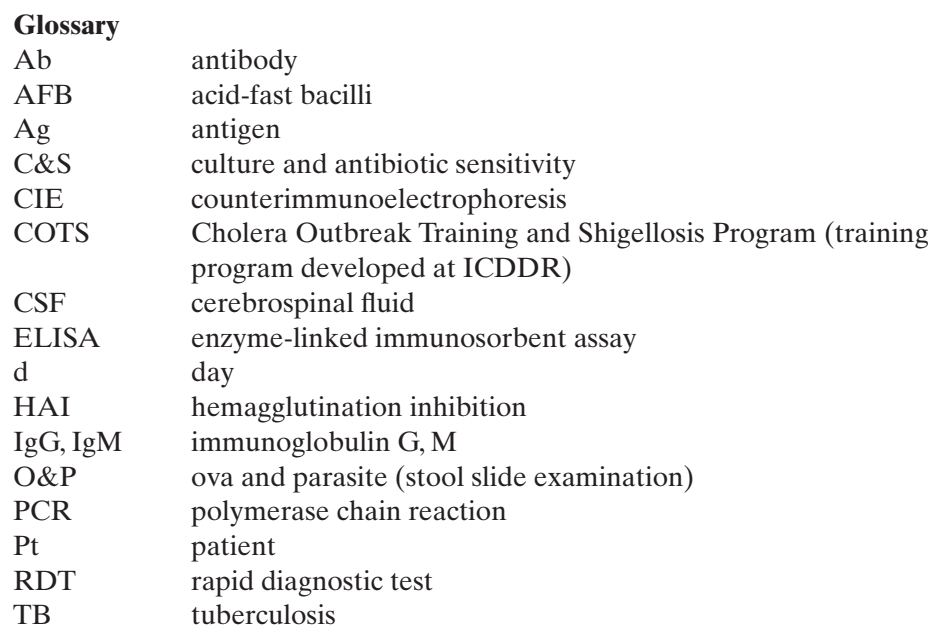

\section{Lab Purposes}

- Confirm syndromic or clinical diagnosis

- Identify treatment options

- Identify control measures

- Characterize the agent (serotype, biotype, antibiogram, etc.)

- Evaluate potential effectiveness of treatment

- Monitor the spread of a particular clone or subtype

- Detect an outbreak and confirm its end

\section{Key Questions on Lab (WHO [1])}

1. Is a laboratory available?

2. What tests does it perform?

3. Is there transport to and from the laboratory?

4. Who prepares transport media?

5. Who provides specimen collection material and supplies?

6. How can these supplies be obtained?

7. Who provides cool packs, transport boxes, car, driver ...?

8. What forms/information must be sent with the specimens?

9. What epidemiological information must accompany test results?

10. How does the epidemiologist obtain results? 
If a lab is not available, then you need a sampling strategy that addresses specimen acquisition, preparation, and transportation in compliance with international regulations on the transport of infectious substances.

Table 7.7.1

Indications, Laboratory Tests, and Expected Availability

\begin{tabular}{|c|c|c|c|}
\hline Cases & Lab Tests & PHC Clinic & $\begin{array}{c}\text { Confirmation } \\
\text { Laboratory }\end{array}$ \\
\hline $\begin{array}{l}\text { Watery diarrhea- } \\
\text { dehydrating } \\
\text { (suspected cholera) }\end{array}$ & $\begin{array}{l}\text { - Fine microscopy for } \\
\text { motility on wet prep, } \\
\text { O\&P } \\
\text { - Gram's stain } \\
\text { - Stool C\&S } \\
\text { - Toxin or } \\
\text { polysaccharide (RDT) }\end{array}$ & $\begin{array}{l}\cdot+ \\
\bullet+ \\
\bullet+1- \\
\bullet ?\end{array}$ & $\begin{array}{l}\text { - District lab } \\
\text { - District lab } \\
\text { - District lab } \\
\text { - District lab }\end{array}$ \\
\hline $\begin{array}{l}\text { Diarrhea with blood } \\
\text { (shigella, salmonella, } \\
\text { amoeba) }\end{array}$ & $\begin{array}{l}\text { - Fine microscopy for } \\
\text { O\&P } \\
\text { - Stool C\&S }\end{array}$ & $\begin{array}{l}\bullet+ \\
\bullet+1-\end{array}$ & $\begin{array}{l}\text { - District lab } \\
\text { - District lab }\end{array}$ \\
\hline $\begin{array}{l}\text { Acute respiratory } \\
\text { infection }\end{array}$ & $\begin{array}{l}\text { - Strep screen, throat } \\
\text { C\&S } \\
\text { - Microscopy with Ziehl- } \\
\text { Neelsen stain for AFB }\end{array}$ & $\begin{array}{l}\bullet+ \\
\bullet+\end{array}$ & $\begin{array}{l}\text { - District lab } \\
\text { - District lab }\end{array}$ \\
\hline $\begin{array}{l}\text { Acute febrile illness } \\
\text { (suspected malaria) }\end{array}$ & $\begin{array}{l}\text { - Microscopy with } \\
\text { Giemsa stain } \\
\text { - Ag detection (RDT) }\end{array}$ & $\begin{array}{l}\bullet+ \\
\bullet+\end{array}$ & $\begin{array}{l}\text { - District lab } \\
\text { - District lab }\end{array}$ \\
\hline $\begin{array}{l}\text { Acute febrile illness } \\
\text { (suspected typhoid) }\end{array}$ & $\begin{array}{l}\text { - } \text { Blood culture } \\
\text { - Serology (ELISA, } \\
\text { RDT) }\end{array}$ & - - & $\begin{array}{l}\text { - District lab } \\
\text { - District lab }\end{array}$ \\
\hline $\begin{array}{l}\text { Meningitis (suspected } \\
\text { meningococcus) }\end{array}$ & $\begin{array}{l}\text { - CSF microscopy with } \\
\text { Gram's stain } \\
\text { - CSF C\&S } \\
\text { - CSF PCR }\end{array}$ & $\begin{array}{l}\bullet+ \\
\bullet--\end{array}$ & $\begin{array}{l}\text { - District lab } \\
\text { - District lab } \\
\text { - District lab }\end{array}$ \\
\hline $\begin{array}{l}\text { Acute jaundice } \\
\text { syndrome (suspected } \\
\text { viral hepatitis) }\end{array}$ & - Serology & $\cdot-$ & - District lab \\
\hline $\begin{array}{l}\text { Acute flaccid } \\
\text { paralysis (suspected } \\
\text { poliomyelitis) }\end{array}$ & $\begin{array}{l}\text { - Virus isolation (cell } \\
\text { culture from stool } \\
\text { specimens) }\end{array}$ & $\bullet-$ & - Reference lab \\
\hline Measles & - Serology (IgM) & $\begin{array}{l}\text { None- } \\
\text { clinical Dx }\end{array}$ & - District lab \\
\hline Neonatal tetanus & None-clinical Dx & $\begin{array}{l}\text { None- } \\
\text { clinical Dx }\end{array}$ & $\begin{array}{l}\text { None-clinical } \\
\text { Dx }\end{array}$ \\
\hline
\end{tabular}




\begin{tabular}{|l|l|l|l|}
\hline \multicolumn{1}{|c|}{ Cases } & \multicolumn{1}{|c|}{ Lab Tests } & PHC Clinic & \multicolumn{1}{c|}{$\begin{array}{c}\text { Confirmation } \\
\text { Laboratory }\end{array}$} \\
\hline $\begin{array}{l}\text { Acute febrile illness } \\
\text { (suspected influenza) }\end{array}$ & $\begin{array}{l}\bullet \text { Ag detection (RDT- } \\
\text { Types A, B) } \\
\bullet \text { PCR }\end{array}$ & $\bullet ?$ & $\bullet$ District lab \\
\hline $\begin{array}{l}\text { Acute febrile illness } \\
\text { (suspected dengue } \\
\text { fever) }\end{array}$ & $\begin{array}{l}\bullet \text { Ab detection (RDT) } \\
\text { immunochromatography } \\
\text { Serology (IgM of paired } \\
\text { sera) } \\
\bullet \text { PCR }\end{array}$ & $\bullet-$ & $\bullet$ District lab \\
\hline Leptospirosis & $\begin{array}{l}\bullet \text { Ab detection (RDT) } \\
- \text { Serology (ELISA) }\end{array}$ & $\bullet-$ & $\bullet$ District lab \\
\hline Typhus & $\bullet$ Serology & $\bullet-$ & $\bullet$ Referencence lab lab \\
\hline
\end{tabular}

Direct techniques

1. microscopy (direct visualization)

2. culture (isolation)

3. immunological Ag techniques-immunohistochemistry, immunochromatography

molecular Ag techniques-DNA or RNA PCR, antigen capture

Indirect serological $(\mathrm{Ab})$ techniques

1. bacterial agglutination

2. hemagglutination inhibition assay

3. ELISA for IgM, IgG

4. microneutralization assay

NB non-specific response from some organisms little or no response in host immunosuppression delays in serological response oblige testing of early and late sera 


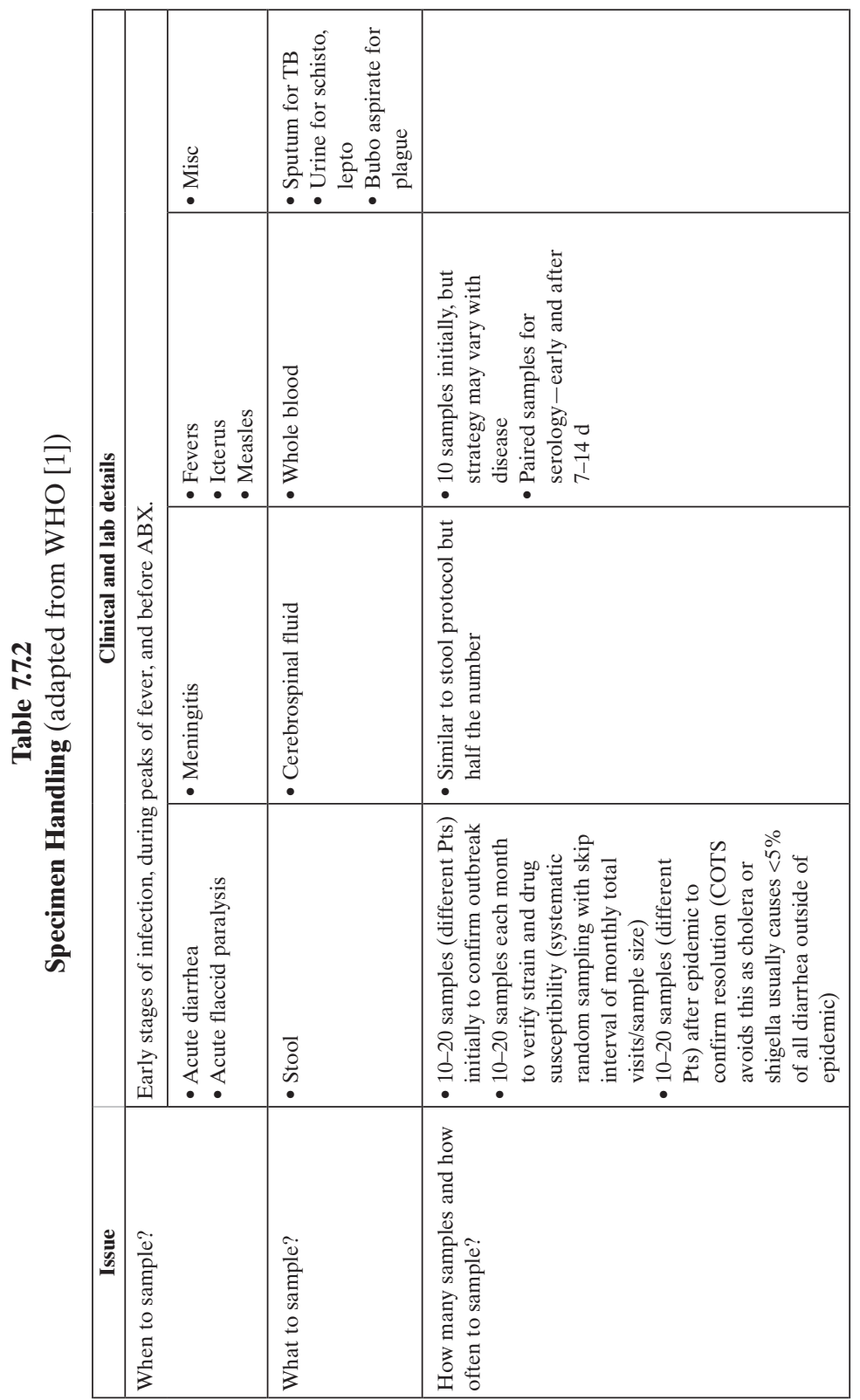


Document $7.7 \quad 279$

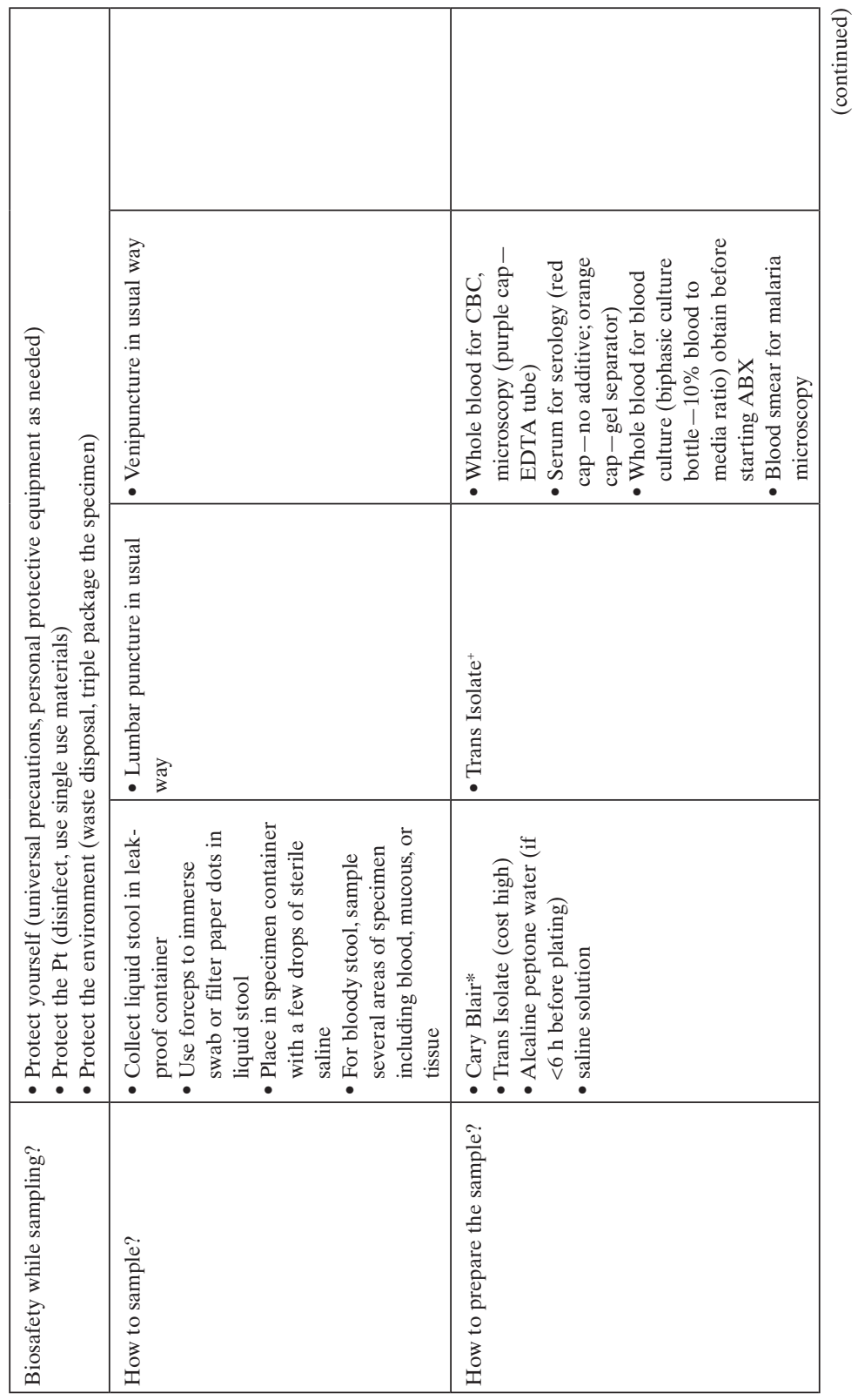




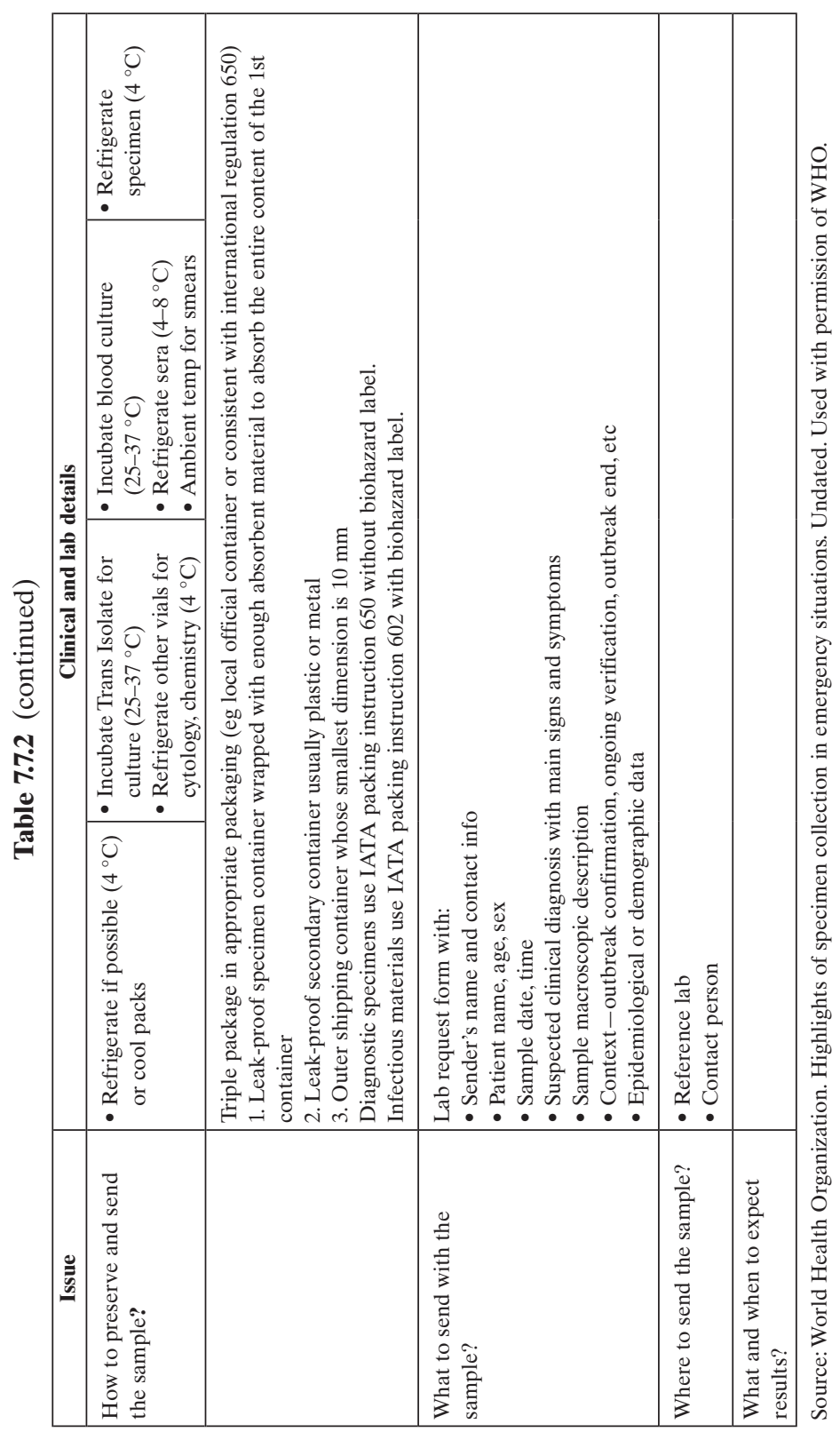




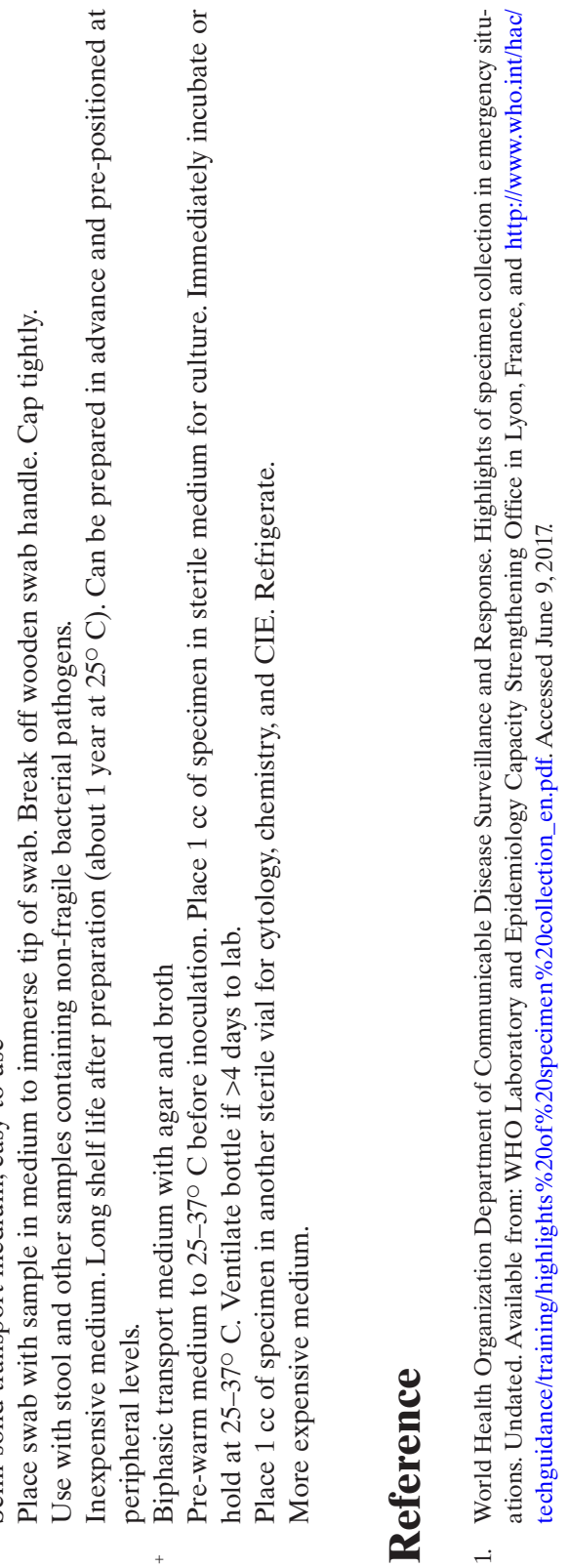




\section{Document 7.8 ACRONYMS}

ABC

ACAPS

ACT

ADB

AfDB

AFRO

AI

AIDS

AKF

ALDAC

ALNAP

AMB E\&P

AMRO

APS

APW

ARI

ART

AU

BCC

BCP

BIC

BMS

BSL

BWI

CA

CAP

CBJ

$\mathrm{CBO}$

CBPF

CBRNE

CCCM

CCS

CCTV

CD

CDC abstain until marriage, be faithful, use condoms

Assessment Capacities Project

artemisinin-based combination therapy

Asian Development Bank

African Development Bank Group

Africa Regional Office (WHO)

avian influenza

acquired immune deficiency syndrome

Aga Khan Foundation

all diplomatic and consular posts (USG)

Active Learning Network for Accountability and Performance (ODI)

Ambassador Extraordinary and Plenipotentiary

Americas Regional Office (WHO)

annual program statement (USAID $\backslash$ OFDA statement of funding opportunities for NGOs)

agreement for the performance of work (WHO contractual work agreement)

acute respiratory infection

antiretroviral therapy

African Union

behavior change communication

business continuity planning

basic internet communications

breast milk substitute

biosafety security level

Bretton Woods Institutions (World Bank and International Monetary Fund)

cooperative agreement

consolidated appeals process (supplanted in 2013 by the humanitarian programme cycle; led by OCHA)

Congressional budget justification

community-based organization

country-based pooled funds (amalgamation of Emergency Response Funds and Common Humanitarian Funds administered by OCHA)

chemical, biological, radiological, nuclear, or explosive

camp coordination and camp management

country cooperation strategy (WHO)

closed-circuit television

communicable diseases

communicable disease control; Centers for Disease Control and Prevention (US) 
CE

CERF

CFE

CHW

CLA

CMAM

CMOC

CMR

$\mathrm{CO}$

COD

CoDel

$\mathrm{COG}$

CONOPS

$\mathrm{CoOp}$

COP

CPA

CRED

CSP

CSW

CTC

CTO

CTP

DA

DANIDA

DART

DCHA

DDR(P)

DDRR(P)

DDRRR(P)

DFID

DHN

DHO

DMAT

DM/HA

DMORT

DO

DOD

DOS

DOTS

DRM complex emergency

Central Emergency Response Fund (administered by OCHA)

Contingency Fund for Emergencies (WHO)

community health worker

cluster lead agency

community-based management of acute malnutrition

civil-military operations center

crude mortality rate

country office (WHO)

common operational dataset

Congressional Delegation

continuity of government

concept of operations

continuity of operations

common operating picture

comprehensive peace agreement

Center for Research on the Epidemiology of Disasters (Belgium)

certified safety professional

commercial sex worker

community therapeutic care (of malnutrition)

cognizant technical officer

cash transfer program

development assistance-US Congress appropriated funds with tight reporting requirements

Danish International Development Agency

Disaster Assistance Response Team (USAID)

Bureau for Democracy, Conflict, and Humanitarian Assistance (USAID)

disarmament, demobilization, and reintegration program

disarmament, demobilization, rehabilitation, and reintegration program

disarmament, demobilization, repatriation, resettlement, and reintegration program

Department for International Development (UK)

digital humanitarian network

District Health Office

Disaster Medical Assistance Team (US)

disaster management and humanitarian assistance

Disaster Mortuary Operational Response Team

designated official (UN)

Department of Defense (US)

Department of State (US)

direct observed therapy short-course (used for TB)

disaster risk management 


\begin{tabular}{|c|c|}
\hline DRR & disaster risk reduction \\
\hline DS & diplomatic security \\
\hline DTM & displacement tracking matrix \\
\hline ECHO & $\begin{array}{l}\text { European Community Humanitarian Office (European } \\
\text { Commission Directorate General for Humanitarian } \\
\text { Aid and Civil Protection which uses ECHO acronym) }\end{array}$ \\
\hline EDG & Emergency Directors' Group \\
\hline EHK & emergency health kit \\
\hline EIA & environmental impact assessment \\
\hline EMRO & Eastern Mediterranean Regional Office (WHO) \\
\hline EMS & Emergency Medical Services \\
\hline EMT & Emergency Medical Team \\
\hline EOC & Emergency Operations Center \\
\hline EOD & explosive ordnance disposal \\
\hline EPI & Expanded Programme on Immunization \\
\hline ERC & Emergency Relief Coordinator (UN) \\
\hline ERF & Emergency Response Framework (WHO) \\
\hline ERT & Emergency Response Team (WHO) \\
\hline ESF & emergency school feeding \\
\hline EST & Emergency Support Team (WHO) \\
\hline ETS & Emergency telecommunications sector \\
\hline EU & European Union \\
\hline EURO & European Regional Office (WHO) \\
\hline EWAR, EWS & early warning system \\
\hline EWARN & Early Warning Alert and Response Network \\
\hline FACT & Field Assessment Coordination Team (IFRC) \\
\hline FAO & Food and Agriculture Organization (UN) \\
\hline FCV & fragility, conflict, and violence \\
\hline FEMA & Federal Emergency Management Agency (US) \\
\hline FETP & Field Epidemiology Training Program \\
\hline FEWS & Famine Early Warning Systems \\
\hline FEWS NET & Famine Early Warning Systems Network \\
\hline FFA & food assistance for assets (formerly food for work) \\
\hline FFP & Food for Peace (USAID $\backslash D C H A$ office) \\
\hline FOG & Field Operations Guide (OFDA) \\
\hline FSN & foreign service national \\
\hline FSO & field security officer \\
\hline FTS & Financial Tracking Service (OCHA) \\
\hline FY & fiscal year \\
\hline G 7 & group of 7 countries \\
\hline G 20 & group of 20 countries \\
\hline G 77 & group of 77 countries \\
\hline GAM & $\begin{array}{l}\text { global acute malnutrition (moderate }+ \text { severe acute } \\
\text { malnutrition) }\end{array}$ \\
\hline GBV & gender-based violence \\
\hline GDACS & Global Disaster Alert and Coordination System \\
\hline GDP & gross domestic product \\
\hline
\end{tabular}




\begin{tabular}{|c|c|}
\hline GEMT & Global Emergency Management Team (WHO) \\
\hline GFATM & Global Fund to Fight AIDS, Tuberculosis and Malaria \\
\hline GFD & general food distribution \\
\hline GFDRR & $\begin{array}{l}\text { Global Facility for Disaster Reduction and Recovery } \\
\text { (WB) }\end{array}$ \\
\hline $\mathrm{GHC}$ & Global Health Cluster \\
\hline GHD & good humanitarian donorship (initiated by DFID) \\
\hline GHSA & Global Health Security Agenda \\
\hline GIC & $\begin{array}{l}\text { Global Impact Charities (consortium of faith-based } \\
\text { organizations involved in HA) }\end{array}$ \\
\hline GIEWS & Global Information and Early Warning System (FAO) \\
\hline GIS & geographic information system \\
\hline GNP & gross national product \\
\hline $\mathrm{GO}$ & governmental organization \\
\hline GOARN & Global Outbreak Alert and Response Network \\
\hline GPN & global private network \\
\hline GTZ & German Technical Cooperation \\
\hline HA & humanitarian assistance \\
\hline HAART & highly active anti-retroviral treatment \\
\hline HAZMAT & hazardous materials \\
\hline HBP & $\begin{array}{l}\text { Health as a Bridge for Peace (WHO headquarters pro- } \\
\text { gram initiative, now discontinued) }\end{array}$ \\
\hline $\mathrm{HC}$ & Humanitarian Coordinator (IASC) \\
\hline $\mathrm{HCC}$ & Health Cluster Coordinator \\
\hline $\mathrm{HCT}$ & Humanitarian Country Team \\
\hline $\mathrm{HCW}$ & health care worker \\
\hline HDR & humanitarian daily ration \\
\hline HELP & $\begin{array}{l}\text { Health Emergencies in Large Populations (ICRC train- } \\
\text { ing course) }\end{array}$ \\
\hline HEPA & high efficiency particulate aspirator (air filter) \\
\hline HeRAMS & Health Resources Availability and Mapping System \\
\hline HEWS & Humanitarian Early Warning System \\
\hline $\mathrm{HH}$ & household \\
\hline HHA & humanitarian health assistance \\
\hline HHS & Department of Health and Human Services (US) \\
\hline $\mathrm{HIC}$ & Humanitarian Information Centre (OCHA) \\
\hline HIS & health information system \\
\hline HIU & Humanitarian Information Unit (US DOS) \\
\hline HIV & human immunodeficiency virus \\
\hline $\mathrm{HNO}$ & humanitarian needs overview \\
\hline HOA & Head of Agency \\
\hline HPAI & highly pathogenic avian influenza \\
\hline HPC & humanitarian programme cycle \\
\hline $\mathrm{HPF}$ & humanitarian pooled fund \\
\hline HRP & humanitarian response plan (akin to SRP) \\
\hline HWCO & Head of WHO Country Office \\
\hline
\end{tabular}


IAHE

IAP

IASC

IATA

IBRD

ICC

ICCG

ICDDR

ICMH

ICRC

ICS

ID

IDA

IDFA

IDLH

IDP

IDSR

IDU

IEC

IED

IEIP

IFRC

IGS

IHL

IHR

IHRL

ILI

IMAI

IMCI

IMF

IMS

INSARAG interagency humanitarian evaluation (IASC process, triggered by L3 declaration, to be done within $12 \mathrm{~m}$ of declaration)

incident action plan

Interagency Standing Committee

International Air Transport Association

International Bank for Reconstruction and Development (World Bank)

International Criminal Court

Intercluster Coordination Group

International Centre for Diarrhoeal Disease Research, Bangladesh

International Centre for Migration and Health

International Committee of the Red Cross

Incident Command System

infectious disease

International Development Association

international disaster and famine account-US Congress appropriated funds which have no Congressional Note reporting requirements (see DA) immediately dangerous to life and health internally displaced person Integrated Disease Surveillance and Response (AFRO initiative expanded to other regions)

injection drug user

information, education, and communication materials improvised explosive device

International Emerging Infections Programs-foreign centers of excellence working in partnership with domestic $\mathrm{MOH}$ and US CDC which train local scientists, provide diagnostic and epi resources in outbreaks, and undertake regional disease control activities

International Federation of Red Cross and Red Crescent Societies

income generating scheme

international humanitarian law

International Health Regulations

international human rights law

influenza-like illness

Integrated Management of Adult Illness (WHO initiative)

Integrated Management of Childhood Illness (WHO initiative)

International Monetary Fund

incident management system

International Search and Rescue Advisory Group 
IO

IOM

IPC

IRA

IRC

IRIN

ISDR

ITN

ITPS

ITWL

IYCFE

JICA

L3

LOU

M\&E

$\mathrm{MCH}$

MCI

MDGs

MDM

MDRO

MEAL

MEB

MEDS

MICS

MIRA

MIS

MISP

$\mathrm{MOH}$

MOSS

MOU

MSF

MUAC

NAF

NATO

NDMA

NDMS

NFI

NGO

NIC

NICS

NID

NIH

NIMA

NRC international organization

International Organization for Migration

Integrated Food Security Phase Classification

initial rapid assessment

International Rescue Committee

Integrated Regional Information Network (OCHA)

International Strategy for Disaster Reduction

insecticide treated nets

insecticide treated plastic sheeting

insecticide treated wall lining

infant and young child feeding in emergencies

Japan International Cooperation Agency

level 3 (system-wide) emergency (supplanted by scale-up process)

letter of understanding

monitoring and evaluation

maternal and child health

mass casualty incident

millennium development goals (supplanted by sustainable development goals)

Médecins du Monde

Mission Disaster Relief Officer (USAID)

monitoring, evaluation, accountability, and learning

minimum expenditure basket

minimum essential datasets

multiple indicator cluster survey

multi-cluster initial rapid assessment

management information system

minimum initial service package (used in reproductive health)

Ministry of Health

minimum operating security standards (UNDSS)

memorandum of understanding

Médecins sans Frontières

mid-upper arm circumference

needs analysis framework

North Atlantic Treaty Organization

National Disaster Management Agency

National Disaster Medical System (US)

non-food item (emergency relief supplies)

non-governmental organization

National Influenza Center

nutritional information in crisis situations

national immunization day

National Institutes of Health (US)

National Imagery and Mapping Agency (US)

Norwegian Refugee Council 


\begin{tabular}{|c|c|}
\hline NRF & National Response Framework (US) \\
\hline NSS & National Security Staff (US) \\
\hline NWOW & new way of working \\
\hline NZODA & New Zealand Official Development Assistance \\
\hline OCHA & $\begin{array}{l}\text { Office for the Coordination of Humanitarian Affairs } \\
\text { (UN) }\end{array}$ \\
\hline ODI & Overseas Development Institute (UK) \\
\hline OECD & $\begin{array}{l}\text { Organization for Economic Cooperation and } \\
\text { Development }\end{array}$ \\
\hline OFDA & Office of US Foreign Disaster Assistance (USAID) \\
\hline OHCHR & $\begin{array}{l}\text { Office of the High Commissioner for Human Rights } \\
\text { (UN) }\end{array}$ \\
\hline OHCT & $\begin{array}{l}\text { Operational Humanitarian Country Team (subnational } \\
\text { level) }\end{array}$ \\
\hline OIE & Office International des Epizooties (World \\
\hline & Organization for Animal Health) \\
\hline OPD & outpatient department \\
\hline OPR & $\begin{array}{l}\text { operational peer review (IASC process, triggered by L3 } \\
\text { declaration, to be done within } 90 \mathrm{~d} \text { of declaration) }\end{array}$ \\
\hline ORS & oral rehydration salts/solution \\
\hline OSCE & Office for Security and Cooperation in Europe \\
\hline OSOCC & On-Site Operations Coordination Center (UN) \\
\hline OVC & orphans and vulnerable children \\
\hline PAHO & $\begin{array}{l}\text { Pan-American Health Organization (precursor to and } \\
\text { functionally synonymous with AMRO) }\end{array}$ \\
\hline PCC & Policy Coordination Committee of the NSC \\
\hline PDD & Presidential Decision Directive \\
\hline PDNA & post-disaster needs assessment \\
\hline PEF & Pandemic Emergency Financing Facility (WB) \\
\hline PEP & post-exposure prophylaxis \\
\hline PEPFAR & President's Emergency Plan for AIDS Relief (US) \\
\hline $\mathrm{PHC}$ & primary health care \\
\hline PHEIC & public health event of international concern (IHR) \\
\hline $\mathrm{PHO}$ & Provincial Health Office \\
\hline $\mathrm{PIO}$ & public international organization \\
\hline $\mathrm{PKO}$ & peace-keeping organization \\
\hline PLWHA & person living with HIV/AIDS \\
\hline РMTCT & prevention of mother-to-child transmission \\
\hline PNG & persona non grata \\
\hline POC & point of contact \\
\hline POLR & provider of last resort \\
\hline PPE & personal protective equipment \\
\hline PPM & $\begin{array}{l}\text { parts per million }=\mathrm{mg} / \mathrm{L}(1 \mathrm{ppm}=1 \mathrm{mg} / \mathrm{L}=.0001 \% \\
\text { active ingredient })\end{array}$ \\
\hline PPRR & prevention, preparedness, response, and recovery \\
\hline PRA & participatory rural appraisal (successor to the RRA) \\
\hline
\end{tabular}




\begin{tabular}{|c|c|}
\hline PRRA & participatory rapid rural appraisal \\
\hline PSC & personal services contractor \\
\hline PSD & protective security detail \\
\hline PSF & Pharmaciens Sans Frontières \\
\hline PVO & private and voluntary organization \\
\hline R2D & relief to development \\
\hline $\mathrm{R} 2 \mathrm{P}$ & responsibility to protect \\
\hline $\mathrm{R} 4$ & $\begin{array}{l}\text { repatriation, reintegration, rehabilitation, and recon- } \\
\text { struction }\end{array}$ \\
\hline $\mathrm{R} \& \mathrm{R}$ & rest and recreation \\
\hline RBM & Roll Back Malaria (WHO program) \\
\hline $\mathrm{RC}$ & Resident Coordinator (UN) \\
\hline REA & rapid epidemiological assessment \\
\hline RFA & request for assistance \\
\hline RFI & request for information \\
\hline RFP & request for proposal \\
\hline RFQ & request for quote \\
\hline $\mathrm{RH}$ & reproductive health \\
\hline RMO & Regional Medical Officer (US Department of State) \\
\hline RMT & Response Management Team (USAID) \\
\hline RNI & $\begin{array}{l}\text { recommended nutritional intake (successor to the rec- } \\
\text { ommended daily allowance (RDA)) }\end{array}$ \\
\hline $\mathrm{RO}$ & regional office \\
\hline RRA & rapid rural appraisal; rapid response account (WHO) \\
\hline RRM & rapid response mechanism \\
\hline RRT & rapid response team \\
\hline RSO & Regional Security Officer (US Department of State) \\
\hline SAG & strategic advisory group \\
\hline SAR & search and rescue \\
\hline SARS & severe acute respiratory syndrome \\
\hline SBU & sensitive but unclassified \\
\hline $\mathrm{SC}$ & Security Council (UN) \\
\hline SCF & Save the Children Foundation \\
\hline SCI & secret compartmented information \\
\hline SDGs & sustainable development goals \\
\hline SEARO & South-East Asia Regional Office (WHO) \\
\hline SGBV & sexual and gender-based violence \\
\hline SHOC & Strategic Health Operations Center (WHO) \\
\hline SMART & $\begin{array}{l}\text { Standardized Monitoring and Assessment of Relief and } \\
\text { Transitions }\end{array}$ \\
\hline SME & subject matter expert \\
\hline SMS & Security Management System (UN) \\
\hline SMT & Security Management Team (UN) \\
\hline SNS & Strategic National Stockpile (US) \\
\hline SO & strategic objective ( $>5$ yrs to achieve) \\
\hline SOD & sudden onset disaster \\
\hline
\end{tabular}




\begin{tabular}{|c|c|}
\hline $\mathrm{SOG}$ & standard operating guideline \\
\hline SOP & standard operating procedure \\
\hline SPHC & selective primary health care \\
\hline SPHERE & $\begin{array}{l}\text { project of humanitarian agencies detailing a humanitar- } \\
\text { ian charter and minimum standards }\end{array}$ \\
\hline $\mathrm{SpO}$ & special objective $(<5$ yrs to achieve $)$ \\
\hline SRP & strategic response plan \\
\hline SRSG & Special Representative of the Secretary General (UN) \\
\hline STI & sexually transmitted infection \\
\hline SUMA & supply management (PAHO/WHO program) \\
\hline SWAP & sector-wide approach \\
\hline TAG & Technical Assistance Group (USAID $\backslash$ OFDA) \\
\hline TB & tuberculosis \\
\hline TBA & traditional birth attendant \\
\hline TFC & therapeutic feeding center \\
\hline TFP & therapeutic feeding program \\
\hline TOR & terms of reference \\
\hline TWG & technical working group \\
\hline UN & United Nations \\
\hline UNAIDS & Joint United Nations AIDS Programme \\
\hline UNCT & UN Country Team \\
\hline UNDAC & UN Disaster Assessment and Coordination team \\
\hline UNDAF & UN Development Assistance Framework \\
\hline UNDMT & UN Disaster Management Team \\
\hline UNDP & UN Development Programme \\
\hline UNDPKO & UN Department of Peacekeeping Operations \\
\hline UNDSS & UN Department of Safety and Security \\
\hline UNEP & UN Environmental Programme \\
\hline UNFPA & UN Fund for Population Activities \\
\hline UNGA & UN General Assembly \\
\hline UNHAS & UN Humanitarian Air Service (WFP) \\
\hline UNHCHR & UN High Commissioner for Human Rights \\
\hline UNHCR & UN High Commissioner for Refugees \\
\hline UNHRD & UN Humanitarian Response Depot \\
\hline UNICEF & UN Children's Fund \\
\hline UNJLC & UN Joint Logistics Center \\
\hline UNPKO & UN Peace-keeping Operations \\
\hline UNRC & UN Resident Coordinator \\
\hline UNSE & UN Special Envoy \\
\hline UNSG & UN Secretary General \\
\hline UNV & UN volunteer \\
\hline USAID & US Agency for International Development \\
\hline USAR & urban search and rescue \\
\hline USCR & US Committee for Refugees \\
\hline USD & US dollar \\
\hline USDA & US Department of Agriculture \\
\hline
\end{tabular}


USG

USPHS

UXO

VAC

VAM

VCT

WASH

WB

WCO

WFP

WHE

WHO

WHZ

WMD

WPRO

WR

WWWW, 4 W
Under Secretary General (UN); US Government US Public Health Service unexploded ordnance Vulnerability Assessment Committee vulnerability assessment and mapping (WFP) voluntary counseling and testing (HIV) water, sanitation, and hygiene World Bank WHO Country Office World Food Programme (UN) WHO Health Emergencies Programme World Health Organization (UN) weight-for-height $\mathrm{z}$ score weapons of mass destruction Western Pacific Regional Office (WHO) WHO Representative (replaced by HWCO) who does what where when (matrix) 